\title{
A discretized Severi-type theorem with applications to harmonic analysis
}

\author{
Joshua Zahl*
}

January 26, 2021

\begin{abstract}
In 1901 , Severi proved that if $Z$ is an irreducible hypersurface in $\mathbb{P}^{4}(\mathbb{C})$ that contains a three dimensional set of lines, then $Z$ is either a quadratic hypersurface or a scroll of planes. We prove a discretized version of this result for hypersurfaces in $\mathbb{R}^{4}$. As an application, we prove that at most $\delta^{-2-\varepsilon}$ direction-separated $\delta$-tubes can be contained in the $\delta$-neighborhood of a lowdegree hypersurface in $\mathbb{R}^{4}$.

This result leads to improved bounds on the restriction and Kakeya problems in $\mathbb{R}^{4}$. Combined with previous work of Guth and the author, this result implies a Kakeya maximal function estimate at dimension $3+1 / 28$, which is an improvement over the previous bound of 3 due to Wolff. As a consequence, we prove that every Besicovitch set in $\mathbb{R}^{4}$ must have Hausdorff dimension at least $3+1 / 28$. Recently, Demeter showed that any improvement over Wolff's bound for the Kakeya maximal function yields new bounds on the restriction problem for the paraboloid in $\mathbb{R}^{4}$.
\end{abstract}

\section{Introduction}

In [19], Severi classified projective hypersurfaces in $\mathbb{P}^{4}(\mathbb{C})$ that contain many lines. Theorem 1.1 (Severi). Let $Z \subset \mathbb{P}^{4}(\mathbb{C})$ be an irreducible hypersurface. Let $\Sigma$ be the set of lines contained in $Z$. Then

- If $\operatorname{dim}(\Sigma)=4$, then $Z$ is a hyperplane.

*University of British Columbia, Vancouver BC. Supported by a NSERC Discovery Grant. 
- If $\operatorname{dim}(\Sigma)=3$, then $Z$ is either a quadratic hypersurface or a scroll of planes.

This theorem was later generalized to higher dimensions by Segre [18]. See [17] for further discussion and [20, Appendix A] for a modern (and English) proof.

Severi's theorem allows us to control the set of directions of lines inside a hypersurface.

Corollary 1.1. Let $Z \subset \mathbb{P}^{4}(\mathbb{C})$ be an irreducible hypersurface. Then the lines contained in $Z$ point in at most a two-dimensional set of directions.

Algebraic varieties containing many lines have recently become a topic of interest when studying the Kakeya and restriction problems. In [12], Katz, Łaba, and Tao observed that the Heisenbeg group

$$
\mathbb{H}=\left\{\left(z_{1}, z_{2}, z_{3}\right) \in \mathbb{C}^{3}: \operatorname{Im}\left(z_{3}\right)=\operatorname{Im}\left(z_{1} \bar{z}_{2}\right)\right\}
$$

is an "almost counter-example" to the Kakeya conjecture - it is a subset of $\mathbb{C}^{3}$ that contains many complex lines, few of which lie in a common plane. In four dimensions, Guth and the author showed in 11] that low-degree hypersurfaces containing many lines are the only possible obstruction to obtaining improved Kakeya estimates in $\mathbb{R}^{4}$. Similar statements are implicit in the works of Guth [10] and Demeter [6].

In this paper, we will prove a discretized version of Theorem 1.1. Our theorem will classify the algebraic hypersurfaces in $\mathbb{R}^{4}$ whose $\delta$-neighborhood, restricted to the unit ball, contains many unit line segments. In contrast to the classical situation studied by Severi, it is not true that if $Z(P)$ is an irreducible hypersurface in $\mathbb{R}^{4}$ whose $\delta$-neighborhood contains many unit line segments, then $Z(P)$ must be a hyperplane, a quadric hypersurface, or a scroll of planes. For example, let $P(x)=x_{1} x_{2}+\delta^{100}$. It is easy to verify that $P$ is irreducible, and the $\delta$-neighborhood of $Z(P)$ contains many unit line segments. Geometrically, $Z(P)$ is a small perturbation of the variety $\left\{x_{1}=0\right\} \cup\left\{x_{2}=0\right\}$, which is a union of two hyperplanes. In particular, large regions of the $\delta$-neighborhoods of $Z(P) \cap B(0,1)$ and $\left\{x_{1}=0\right\} \cup\left\{x_{2}=0\right\} \cap B(0,1)$ are comparable. In the example above, "half" of the unit line segments lying near $N_{\delta}(Z)$ are contained in the $\delta$-neighborhood of the hyperplane $\left\{x_{1}=0\right\}$, and "half" are contained in the $\delta$-neighborhood of the hyperplane $\left\{x_{2}=0\right\}$.

As a more extreme example, $Z(P)$ might be a small perturbation of the variety $Z_{1} \cup Z_{2} \cup Z_{3} \cup Z_{4}$, where $Z_{1}$ is an arbitrary low-degree hypersurface containing few lines; $Z_{2}$ is a scroll of planes; $Z_{3}$ is a hyperplane; and $Z_{4}$ is a quadratic hypersurface. Our discretized version of Severi's theorem follows this idea. Informally it says that if $Z$ is a hypersurface, then the unit line segments contained in $N_{\delta}(Z)$ can be partitioned into four classes in the spirit of the above example. 
As an application of our techniques, we prove a variant of Corollary 1.1, which says that the set of unit line segments in the unit ball lying near $N_{\delta}(Z)$ can point in at most $\delta^{-2-\varepsilon}$ different $\delta$-separated directions. As discussed in Section 10, this result yields an improved bound for the Kakeya maximal function in $\mathbb{R}^{4}$. In $|5|$, Demeter proved that such an improvement for the Kakeya maximal function would yield new bounds on the restriction problem for the paraboloid in $\mathbb{R}^{4}$. This will be discussed further in Section 1.2 .

Before stating the main result of this paper, we will introduce some notation.

Definition 1.1. Let $\mathcal{L}$ be the set of lines in $\mathbb{R}^{4}$. For each $\ell \in \mathcal{L}$, define $\operatorname{Dir}(\ell)$ to be a unit vector in $\mathbb{R}^{4}$ pointing in the same direction as $\ell$. By convention, we will choose the unit vector $v=\left(v_{1}, v_{2}, v_{3}, v_{4}\right)$ with $v_{1} \geq 0 ; v_{2} \geq 0$ if $v_{1}=0 ; v_{3} \geq 0$ if $v_{1}=v_{2}=0$; and $v_{4}=1$ if $v_{1}=v_{2}=v_{3}=0$.

If $E$ is a set of unit vectors in $\mathbb{R}^{4}$ and $\delta>0$, we will write $\mathcal{E}_{\delta}(E)$ to denote the $\delta$ covering number of $E$. More generally if $(X, d)$ is a metric space and $E \subset X, \mathcal{E}_{\delta}(E)$ will denote the $\delta$ covering number of $E$.

Our proof will refer to "rectangular prisms," which are the discretized analogues of lines, planes, and hyperplanes. These prisms will have "long directions," which have length two, and "short directions," which have length much smaller than two. Informally, we say a rectangular prism is $k$ dimensional if it has $k$ long directions (all such prisms will be contained in $\mathbb{R}^{4}$ ). We say that a line is covered by a rectangular prism if the intersection has length at least two.

The following is a discretized version of Theorem 1.1.

Theorem 1.2. Let $P \in \mathbb{R}\left[x_{1}, x_{2}, x_{3}, x_{4}\right]$ be a polynomial of degree $D$, and let $Z=$ $Z(P) \cap B(0,1)$. Let $\delta, \kappa, u, s \in(0,1)$ be numbers satisfying $0<\delta<u<s<1$ and $\delta<\kappa<1$ (if these conditions are not satisfied the theorem is still true, but it has no content).

Define

$$
\Sigma=\left\{\ell \in \mathcal{L}:\left|\ell \cap N_{\delta}(Z)\right| \geq 1\right\}
$$

Then we can write $\Sigma=\Sigma_{1} \cup \Sigma_{2} \cup \Sigma_{3} \cup \Sigma_{4}$, where

- There is a collection of $O_{D}\left(|\log \delta|^{O(1)} s^{-2}\right)$ rectangular prisms of dimensions $2 \times s \times s \times s$ so that every line from $\Sigma_{1}$ is covered by one of these prisms.

- There is a collection of $O_{D}\left((|\log \delta| / s)^{O(1)} u^{-1}\right)$ rectangular prisms of dimensions $2 \times 2 \times u \times u$ so that every line from $\Sigma_{2}$ is covered by one of these prisms.

- There is a collection of $O_{D}\left(|\log \delta|^{O(1)}\right)$ rectangular prisms of dimensions $2 \times$ $2 \times 2 \times \kappa$ so that every line in $\Sigma_{3}$ is covered by one of these prisms. 
- There is a set $\Sigma_{4}^{\prime} \subset \Sigma_{4}$ with

$$
\mathcal{E}_{\delta}\left(\Sigma_{4}^{\prime}\right) \gtrsim_{D}(u s \kappa /|\log \delta|)^{O(1)} \mathcal{E}_{\delta}\left(\Sigma_{4}\right)
$$

and a quadratic hypersurface $Q$ so that for every line $\ell^{\prime} \in \Sigma_{4}^{\prime}$, there is a line $\ell$ contained in $Z(Q)$ with

$$
\operatorname{dist}\left(\ell, \ell^{\prime}\right) \lesssim_{D}(|\log \delta| /(u s \kappa))^{O(1)} \delta .
$$

We will prove Theorem 1.2 (or actually a slightly more technical generalization) in Section 8 below. As a corollary of (the more technical version of) Theorem 1.2, we obtain the following discretized analogue of Corollary 1.1 .

Corollary 1.2 (Few directions near a low-degree variety). Let $P \in \mathbb{R}\left[x_{1}, \ldots, x_{4}\right]$ be a polynomial of degree $D$ and let $Z=Z(P) \cap B(0,1)$. Let $0<\delta<1$ and define

$$
\Sigma=\left\{\ell \in \mathcal{L}:\left|\ell \cap N_{\delta}(Z)\right| \geq 1\right\} .
$$

Then for each $\varepsilon>0$,

$$
\mathcal{E}_{\delta}(\operatorname{Dir}(\Sigma)) \lesssim_{D, \varepsilon} \delta^{-2-\varepsilon} .
$$

In brief, Corollary 1.2 follows from Theorem 1.2 by choosing $s=|\log \delta|^{-C_{1}}, u=$ $|\log \delta|^{-C_{2}}, \kappa=|\log \delta|^{-C_{3}}$, where $C_{1}, C_{2}, C_{3}$ are large absolute constants. Since the lines contained in a quadratic hypersurface in $\mathbb{R}^{4}$ can point in few directions, the set of directions of lines in $\Sigma_{4}^{\prime}$ is small. Using a slightly more technical version of Theorem 1.2. we can also guarantee that the set of directions of lines in $\Sigma_{4}$ is small. The lines in $\Sigma_{1}, \Sigma_{2}$, and $\Sigma_{3}$ are handled by re-scaling and induction on scales. Corollary 1.2 will be proved in detail in Section 9 .

Remark 1.1. We could replace the condition $\left|\ell \cap N_{\delta}(Z)\right| \geq 1$ in the definition of $\Sigma$ with $\left|\ell \cap N_{\delta}(Z)\right| \geq c$ for any fixed constant $c>0$. Then the implicit constant in (1) would also depend on $c$.

In [10], Guth stated the following conjecture

Conjecture 1.1. Let $Z \subset \mathbb{R}^{d}$ be a m-dimensional variety defined by polynomials of degree at most $D$, and let $\Sigma$ be the set of lines in $\mathbb{R}^{d}$ satisfying $\left|\ell \cap N_{\delta}(Z) \cap B(0,1)\right| \geq 1$. Then for each $\delta>0$ and $\varepsilon>0$, the set of directions of lines in $\Sigma$ can be covered by $O_{d, D, \varepsilon}\left(\delta^{1-m}\right)$ balls of radius $\delta$.

When $m=2$, Conjecture 1.1 is straightforward, and the result was used by Guth in $[9]$ to obtain improved restriction estimates in $\mathbb{R}^{3}$. Corollary 1.2 proves Conjecture 1.1 in the case $d=4, m=3$. For $m \geq 4$ the conjecture remains open.

Addendum added April 2018: Conjecture 1.1 was recently proved in all dimensions by Katz and Rogers [13]. 


\subsection{Progress on the Kakeya conjecture}

Recall the Kakeya maximal function conjecture. In the statement below, a $\delta$-tube is the $\delta$-neighborhood of a unit line segment.

Conjecture 1.2. Let $\mathbb{T}$ be a set of $\delta$-tubes in $\mathbb{R}^{d}$ that point in $\delta$-separated directions. Then for each $\varepsilon>0$,

$$
\left\|\sum_{T \in \mathbb{T}} \chi_{T}\right\|_{p^{\prime}} \lesssim \varepsilon \delta^{1-d / p-\varepsilon}, \quad p=d
$$

Conjecture 1.2 has been proved when $d=2$ by Córdoba [4] and remains open for $d \geq 3$. If the exponent $p=d$ in (2) is replaced by a number $1 \leq p \leq d$, then (2) is called a Kakeya maximal function estimate in $\mathbb{R}^{d}$ at dimension $p$.

Using the results of Guth and the author from [11], Theorem 1.2 can be used to obtain a Kakeya maximal function estimate in $\mathbb{R}^{4}$ at dimension $3+1 / 28$.

Theorem 1.3. Let $\mathbb{T}$ be a set of $\delta$-tubes in $\mathbb{R}^{4}$ that point in $\delta$-separated directions. Then for each $\varepsilon>0$,

$$
\left\|\sum_{T \in \mathbb{T}} \chi_{T}\right\|_{p^{\prime}} \lesssim \varepsilon \delta^{1-4 / p-\varepsilon}, \quad p=3+1 / 28 .
$$

Corollary 1.3. Every Besicovitch set in $\mathbb{R}^{4}$ has Hausdorff dimension at least $3+$ $1 / 28$.

Theorem 1.3 will be proved in Section 10, It is an improvement over the previous bound $p=3$, which was due to Wolff [22]. Previously, Łaba and Tao [15] proved that every Besicovitch set in $\mathbb{R}^{4}$ must have upper Minkowski dimension at least $3+\varepsilon_{0}$ for some positive constant $\varepsilon_{0}>d^{1}$. In a similar vein, Tao [21] proved that every Kakeya set in $\mathbb{F}_{p}^{4}$ must have cardinality at least $c p^{3+1 / 16}$. This was later improved by Dvir 6 and then Dvir, Kopparty, Saraf, and Sudan [7], who proved nearly sharp bounds on the size of Kakeya sets in $\mathbb{F}_{p}^{n}$ for every $n$.

\subsection{Progress on the restriction conjecture}

Let $f:[-1,1]^{d-1} \rightarrow \mathbb{C}$. For each $x=\left(\underline{x}, x_{d}\right) \in \mathbb{R}^{d}$, define the extension operator $E f$ by

$$
E f(x)=\int_{[-1,1]^{d-1}} f(\xi) e^{\xi \cdot \underline{x}+|\xi|^{2} x_{n}} d \xi .
$$

The restriction conjecture for the paraboloid relates the size of $f$ and $E f$.

\footnotetext{
${ }^{1}$ Without attempting to optimize their arguments, Łaba and Tao obtained the estimate $\varepsilon_{0} \geq$ $2^{-30}$. A careful analysis of their methods would likely yield a larger value of $\varepsilon_{0}$.
} 
Conjecture 1.3. For each $q>\frac{2 d}{d-1}$ and each $f:[-1,1]^{d} \rightarrow \mathbb{C}$, we have

$$
\|E f\|_{q} \lesssim q, d\|f\|_{\infty} \text {. }
$$

Conjecture 1.3 has been proved when $d=2$ by Fefferman and Zygmund 8, 25]. For $d \geq 2$ the problem remains open; the current best bounds are due to Guth $[9,10]$. When $d=4$, (4) is known for $q>14 / 5$. In [6], Demeter proved that improvements to the Kakeya maximal function conjecture in $\mathbb{R}^{4}$ would lead to progress on the restriction conjecture.

Theorem 1.4 ( [6], Theorem 1.4). Let $d=4$. If (2) holds for some $p>3$, then (4) holds for some $q<14 / 5$.

When Theorems 1.3 and 1.4 are combined, they yield an improved restriction estimate for the paraboloid in $\mathbb{R}^{4}$. Inserting the exponent $p=3+1 / 28$ into Demeter's argument yields the exponent $q=\frac{14}{5}-\frac{2}{416515}$.

\subsection{Proof sketch}

In this section we will survey the main ideas in the proof of Theorem 1.2. Let $P \in \mathbb{R}\left[x_{1}, \ldots, x_{4}\right]$ be a polynomial of degree at most $D$ and let $Z=Z(P) \cap B(0,1)$. We wish to understand the set of lines that satisfy $\left|\ell \cap N_{\delta}(Z)\right| \geq 1$.

For the purposes of this sketch, we will assume that $\nabla P(z) \sim 1$ for all $z \in Z$ and that $|P(x)| \leq \delta$ for all $x \in N_{\delta}(Z)$. While these assumptions certainly need not hold in general, a reduction performed in Section 3 allows us to reduce to the case where a similar (though slightly more technical) statement holds.

Let $\ell$ be a line satisfying $\left|\ell \cap N_{\delta}(Z)\right| \geq 1$ and let $x \in \ell \cap N_{\delta}(Z)$. Let $\ell(t)$ be a unit speed parameterization of $\ell$ with $\ell(0)=x$. Then $P(\ell(t))$ is a univariate polynomial of degree at most $D$, and $|P(\ell(t))|$ is small for many values of $t$. More precisely, we have

$$
|\{t \in[-1,1]:|P(\ell(t))| \lesssim \delta\}| \gtrsim 1
$$

This means that all of the coefficients of $P(\ell(t))$ have magnitude $\lesssim \delta$ (the implicit constant may depend on $D$, but we will suppress this dependence here). In particular, if $v$ is a unit vector pointing in the same direction as $\ell$ and if $z \in Z$ satisfies $\operatorname{dist}(x, z) \leq \delta$, then

$$
|v \cdot \nabla P(z)| \lesssim \delta \quad \text { and } \quad\left|(v \cdot \nabla)^{2} P(z)\right| \lesssim \delta
$$

For each $z \in Z(P)$, the set of vectors $\left\{v \in S^{3}: v \cdot \nabla P(z)=0,(v \cdot \nabla)^{2} P(z)=0\right\}$ is called the quadratic cone of $Z(P)$ at $z$, and it is closely related to the second fundamental form of $Z(P)$ at $z$. 
We wish to understand the relationship between the set of vectors satisfying (5) and the set of vectors in the quadratic cone of $Z(P)$ at $z$. It thus seems reasonable to ask: if $z \in Z$ and if a unit vector $v \in S^{3}$ satisfies (5), must it be the case that $v$ is contained in the $\lesssim \delta$-neighborhood of the quadratic cone of $Z(P)$ at $z$ ?

In short, the answer is no. As a first example, consider the polynomial $P_{1}\left(x_{1}, x_{2}\right.$, $\left.x_{3}, x_{4}\right)=x_{1}+\delta x_{2}^{2}$, and let $z=0$. Then the quadratic cone of $Z\left(P_{1}\right)$ at $z=0$ is $\left\{\left(v_{1}, v_{2}, v_{3}, v_{4}\right) \in S^{3}: v_{1}=v_{2}=0\right\}$. However, the set of vectors satisfying (5) is much larger; it is comparable to $\left\{\left(v_{1}, v_{2}, v_{3}, v_{4}\right) \in S^{3}:\left|v_{1}\right| \lesssim \delta\right\}$. In this example, the $\delta$ neighborhood of $Z\left(P_{1}\right) \cap B(0,1)$ is comparable to the $\delta$-neighborhood of a hyperplane. In Section 4 we will expand on this observation. We will prove a technical variant of the following idea: if the coefficients of the second fundamental form of $Z(P)$ are all very small at a typical point, then large pieces of $Z(P) \cap B(0,1)$ can be contained in the thin neighborhood of a hyperplane. The lines having large intersection with these pieces will end up in the set $\Sigma_{3}$ from the statement of the theorem.

As a second example, consider the polynomial $P_{2}\left(x_{1}, x_{2}, x_{3}, x_{4}\right)=x_{1}+x_{2}^{2}$ and let $z=0$. Then the quadratic cone of $Z\left(P_{2}\right)$ at $z=0$ is again $\left\{\left(v_{1}, v_{2}, v_{3}, v_{4}\right) \in S^{3}: v_{1}=\right.$ $\left.v_{2}=0\right\}$, while the set of vectors satisfying (5) is comparable to $\left\{\left(v_{1}, v_{2}, v_{3}, v_{4}\right) \in\right.$ $\left.S^{3}:\left|v_{1}\right| \lesssim \delta,\left|v_{2}\right| \lesssim \delta^{1 / 2}\right\}$. In this example, at least one coefficient of the second fundamental form of $Z\left(P_{2}\right)$ at $z=0$ has large magnitude, and the quadratic cone of $Z\left(P_{2}\right)$ at $z=0$ is a plane. In Section 6, we will show that if at least one coefficient of the second fundamental form of $Z(P)$ is large at a typical point, and if the quadratic cone is comparable to either a plane or a union of two planes, then most of the lines contained in $N_{\delta}(Z(P) \cap B(0,1))$ can be covered by a union of one and two dimensional prisms. These lines will end up in the sets $\Sigma_{1}$ and $\Sigma_{2}$ from the statement of the theorem.

Now let $z \in Z(P) \cap B(0,1)$ and suppose that at least one coefficient of the second fundamental form of $Z(P)$ at $z$ is large and that the quadratic cone of $Z(P)$ at $z$ is not comparable to either a plane or a union of two planes. Then the set of vectors satisfying (5) is comparable to the $\delta$-neighborhood of the quadratic cone of $Z(P)$ at $z$. In Section 7, we will show that if this happens at a typical point, then morally speaking $Z(P)$ must be a quadratic hypersurface. More precisely, many of the lines lying near $N_{\delta}(Z(P) \cap B(0,1))$ are almost contained in a quadratic hypersurface. These lines will be end up in the set $\Sigma_{4}$ from the statement of the theorem.

\subsection{Thanks}

The author would like to thank Larry Guth and Ciprian Demeter for helpful discussions. 


\section{A primer on real algebraic geometry}

Our proof will use several facts about semi-algebraic sets. Further background can be found in [2]. A semi-algebraic set is a boolean combination of sets of the form

$$
S=\left\{x \in \mathbb{R}^{d}: P_{1}(x)=0, \ldots, P_{k}(x)=0, Q_{1}(x)>0, \ldots, Q_{\ell}(x)>0\right\},
$$

where $P_{1}, \ldots, P_{k}$ and $Q_{1}, \ldots, Q_{\ell}$ are polynomials. We define the complexity of $S$ to be the minimum of $\operatorname{deg}\left(P_{1}\right)+\ldots+\operatorname{deg}\left(P_{k}\right)+\operatorname{deg}\left(Q_{1}\right)+\ldots+\operatorname{deg}\left(Q_{\ell}\right)$, where the minimum is taken over all representations of $S$ of the form (6). We define the complexity of a semi-algebraic set to be the sum of the complexities of its constituent components of the form (6) above.

If $S, T \subset \mathbb{R}^{d}$ are semi-algebraic sets of complexity $E_{1}$ and $E_{2}$ respectively, then $S \cup T, S \cap T$, and $S \backslash T$ are semi-algebraic sets of complexity $O_{d, E_{1}, E_{2}}(1)$. If $\pi: \mathbb{R}^{d} \rightarrow \mathbb{R}^{e}$ is a projection, then $\pi(S)$ is a semi-algebraic set of complexity $O_{d, E_{1}}(1)$.

If $S \subset \mathbb{R}^{d}, T \subset \mathbb{R}^{e}$ are semi-algebraic sets, we say that a function $f: S \rightarrow T$ is semi-algebraic of complexity $E$ if the graph of $f$ is a semi-algebraic set of complexity $E$. Clearly if $f: S \rightarrow T$ is a semi-algebraic bijection of complexity $E$, then $f^{-1}: T \rightarrow$ $S$ is also a semi-algebraic bijection of complexity $E$.

If $S \subset \mathbb{R}^{d}$ is a semi-algebraic set, we define its $\operatorname{dimension} \operatorname{dim}(S)$ to be the largest integer $e$ so that there is a subset $S^{\prime} \subset S$ that is homeomorphic to the open $e$ dimensional cube $(0,1)^{e}$. If $S \subset \mathbb{R}^{d}$ has dimension $e$ and complexity $E$, then there is an $e$-dimensional real algebraic variety $S \subset Z \subset \mathbb{R}^{d}$ that is defined by polynomials of degree $O_{d, E}(1)$. If $S$ and $T$ are semi-algebraic sets, and if there is a semi-algebraic bijection $f: S \rightarrow T$, then $S$ and $T$ have the same dimension.

Occasionally, we will refer to semi-algebraic subsets of the sphere $S^{d}$ or the affine Grassmannian $\operatorname{Grass}(d ; e)$ of e-dimensional affine subspaces of $\mathbb{R}^{d}$. To do this, we will identify $S^{d}$ or $\operatorname{Grass}(d ; e)$ with a semi-algebraic set in $\mathbb{R}^{N}$ for some $N=O_{d}(1)$.

In the remainder of this section, we will list several standard results about real algebraic sets that will be used throughout the proof.

Lemma 2.1 (Milnor-Thom theorem). Let $S \subset \mathbb{R}^{d}$ be a semi-algebraic set of complexity at most $E$. Then $S$ has $O_{E, d}(1)$ connected components.

This is a variant of the Milnor-Thom Theorem [16,21]. See Barone-Basu [1] for the above formulation.

Lemma 2.2 (Wongkew $[23]$ ). Let $Z \subset \mathbb{R}^{d}$ be a real algebraic variety of dimension e that is defined by polynomials of degree at most $D$. Then for each $u>0$, we have

$$
\left|N_{u}(Z \cap B(0,1))\right| \leq \sum_{j=0}^{e} C_{d, j}(D u)^{d-e},
$$


where the numbers $C_{d, j}$ are constants depending only on $d$ and $j$.

Corollary 2.1. Let $S \subset B(0,1) \subset \mathbb{R}^{d}$ be a semi-algebraic set of dimension e and complexity at most $E$. Then for each $u>0$, we have

$$
\left|N_{u}(S)\right| \lesssim_{E, d} u^{d-e}
$$

Since $\mathcal{E}_{u}\left(N_{w}(S)\right) \lesssim u^{-d}\left|N_{u+w}(S)\right|$, we obtain the following corollary.

Lemma 2.3 (Covering number of neighborhoods of semi-algebraic sets). Let $S \subset$ $B(0,1) \subset \mathbb{R}^{d}$ be a semi-algebraic set of dimension e and complexity at most $E$. Then for each $u>0$, we have

$$
\mathcal{E}_{u}\left(N_{u}(S)\right) \lesssim_{E, d} u^{-e}
$$

If $0<u<w$, then

$$
\mathcal{E}_{u}\left(N_{w}(S)\right) \lesssim_{E, d} u^{-d} w^{d-e}=u^{-e}(w / u)^{e-d}
$$

A similar result can be found in [24, Theorem 5.9]. The next lemma is a quantitative version of the statement that every connected smooth semi-algebraic set is path connected.

Lemma 2.4. Let $S \subset \mathbb{R}^{d}$ be a semi-algebraic set of complexity at most $E$ and diameter at most one. Suppose as well that $S$ is a connected smooth manifold. Then any two points in $S$ can be connected by a smooth curve of length $O_{d, E}(1)$.

We will also need the following multi-dimensional Remez-type inequality. See, e.g. [3, Theorem 2].

Lemma 2.5. Let $P \in \mathbb{R}\left[x_{1}, \ldots, x_{d}\right]$ be a polynomial of degree at most $D$. Let $\Omega \subset \mathbb{R}^{d}$ be an open convex set. Let $m=\sup _{x \in \Omega}|P(x)|$. Then for each $0<\lambda<1$,

$$
|\{x \in \Omega:|P(x)| \leq \lambda m\}| \leq 4 d|\Omega| \lambda^{1 / D} .
$$

Corollary 2.2. Let $P \in \mathbb{R}\left[x_{1}, \ldots, x_{d}\right]$ be a homogeneous polynomial of degree $D$. Let $m=\sup _{|x|=1}|P(x)|$. Then for each $0<\lambda<1$,

$$
\mu_{\sigma}\left(\left\{x \in S^{d-1}:|P(x)| \leq \lambda m\right\}\right) \lesssim_{d} \lambda^{1 / O_{D}(1)}
$$

where $\mu_{\sigma}$ is the Haar probability measure on the sphere. 
Lemma 2.6 (Selecting a point from each fiber). Let $X \subset \mathbb{R}^{d}$ be a semi-algebraic set and let $f: X \rightarrow Y$ be a semi-algebraic map. Suppose that both $X$ and $f$ have complexity at most $E$. Then there is a semi-algebraic set $X^{\prime} \subset X$ of complexity $O_{E, d}(1)$ so that $f\left(X^{\prime}\right)=f(X)$ and $f: X^{\prime} \rightarrow Y$ is an injection.

Proof. Define a semi-algebraic ordering ">" on $\mathbb{R}^{d}$ with the following properties. (A): If $x, x^{\prime} \in \mathbb{R}^{d}$ then exactly one of the following holds: $x>x^{\prime}, x=x^{\prime}$, or $x<x^{\prime}$. (B): The set

$$
O=\left\{\left(x, x^{\prime}\right) \in \mathbb{R}^{d} \times \mathbb{R}^{d}: x<x^{\prime}\right\}
$$

is semi-algebraic of complexity $O_{d}(1)$.

An example of such an ordering is the lexicographic order on $x=\left(x_{1}, \ldots, x_{d}\right)$. Observe that

$$
\left\{\left(x, x^{\prime}\right) \in X \times X: f(x)=f\left(x^{\prime}\right)\right\}
$$

is semi-algebraic of complexity $O_{E}(1)$. Thus

$$
B=\left\{\left(x, x^{\prime}\right) \in X \times X: f(x)=f\left(x^{\prime}\right), x<x^{\prime}\right\}
$$

is semi-algebraic of complexity $O_{E, d}(1)$. Let $\pi: X \times X \rightarrow X$ be the projection to the first coordinate. Then

$$
X^{\prime}=X \backslash \pi(B)=\left\{x \in X: x \geq x^{\prime} \forall x^{\prime} \in X \text { with } f(x)=f\left(x^{\prime}\right)\right\}
$$

is semi-algebraic of complexity $O_{E, d}(1)$. Note that $f\left(X^{\prime}\right)=f(X)$ and that $f: X^{\prime} \rightarrow$ $Y$ is an injection. Indeed, if $x, x^{\prime} \in W$ with $f(x)=f\left(x^{\prime}\right)$ then $x \geq x^{\prime}$ and $x^{\prime} \geq x$, which implies $x=x^{\prime}$.

\section{$2.1 \quad$ Bundles of lines}

The map $\ell \mapsto \operatorname{Dir}(\ell)$ described in Definition 1.1 is badly behaved for lines lying in (or near) the hyperplane $x_{1}=0$. To avoid this issue, we will only consider lines $\ell \in \mathcal{L}$ that make a small angle with the $e_{1}$ direction. Abusing notation slightly, we will re-define $\mathcal{L}$ to be the set of lines in $\mathbb{R}^{4}$ that make an angle $\leq 1 / 10$ with the $e_{1}$ direction.

Definition 2.1. For $Z \subset \mathbb{R}^{4}$ and $0<\delta<c$, define

$$
\Sigma_{\delta, c}(Z)=\left\{\ell \in \mathcal{L}:\left|\ell \cap N_{\delta}(Z)\right| \geq c\right\} .
$$

Define $\Sigma_{\delta}(Z)=\Sigma_{\delta, 1}(Z)$. 
Let $\ell \in \mathcal{L}$. Define $v(\ell)$ to be the unit vector $v \in \mathbb{R}^{4}$ that points in the same direction as $\ell$ and satisfies $\angle\left(v, e_{1}\right) \leq 1 / 10$.

Definition 2.2. Let $\Sigma \subset \mathcal{L}$ be a set of lines. For each $x \in \mathbb{R}^{4}$, define

$$
\Sigma(x)=\{\ell \in \Sigma: x \in \ell\} .
$$

Definition 2.3. Let $Z \subset \mathbb{R}^{4}$ and let $\Sigma \subset \mathcal{L}$ be a set of lines. Define

$$
\Gamma(Z, \Sigma)=\{(x, \ell) \in Z \times \Sigma: x \in \ell\} .
$$

If $\Gamma \subset \Gamma(Z, \Sigma)$, then for each $x \in Z$ define

$$
\Gamma(x)=\{\ell \in \mathcal{L}:(x, \ell) \in \Gamma\},
$$

and for each $\ell \in \Sigma$, define

$$
\Gamma(\ell)=\left\{x \in \mathbb{R}^{4}:(x, \ell) \in \Gamma\right\} .
$$

\section{Replacing $P$ by a better-behaved polynomial}

In this section we will perform a convenient technical reduction. Informally speaking, this reduction lets us assume that the polynomial $P$ from the statement of Theorem 1.2 obeys the bounds

$$
\begin{array}{ll}
|\nabla P(x)| \sim 1 & \text { for all } x \in Z(P) \cap B(0,1), \\
|P(x)| \lesssim \delta & \text { for all } x \in N_{\delta}(Z(P)) \cap B(0,1) .
\end{array}
$$

If (9) were true, it would yield a lot of useful information about the unit line segments contained in $N_{\delta}(Z(P))$. For example, if $\ell \in \Sigma_{\delta}(Z)$ and $x \in Z(P) \cap N_{\delta}(\ell) \cap B(0,1)$, then $v(\ell)$ is almost contained in the tangent plane $T_{x}(Z(P))$. While $(9)$ need not be true, the following proposition will still allow us to recover some of the useful consequences of (9).

Proposition 3.1. Let $P$ be a polynomial of degree at most $D$. Let $Z=Z(P) \cap$ $B(0,1)$, let $\delta>0$, and let $\Sigma \subset \Sigma_{\delta}(Z)$ be a semi-algebraic set of complexity at most $E$. Then there exist sets $\Sigma_{1}, \ldots, \Sigma_{b}$; polynomials $P_{1}, \ldots, P_{b}$, and sets $\Gamma_{1}, \ldots, \Gamma_{b}$ so that the following holds.

1. $b \lesssim_{D, E}|\log \delta|$. 
2. Each $P_{j}$ has degree at most D. Each set $\Sigma_{j}$ and $\Gamma_{j}$ is semi-algebraic of complexity $O_{D, E}(1)$.

3. $\Gamma_{j} \subset \Gamma\left(N_{\delta}\left(Z_{j}\right), \Sigma_{j}\right)$, where

$$
Z_{j}=\left\{x \in Z\left(P_{j}\right) \cap B(0,1): 1 \leq\left|\nabla P_{j}(x)\right| \leq 2\right\} .
$$

4. $\Sigma=\bigcup_{j=1}^{b} \Sigma_{j}$

5. For each $\ell \in \Sigma_{j}$, we have $\left|\Gamma_{j}(\ell)\right| \gtrsim_{D}|\log \delta|^{-1}$.

6. For each $(x, \ell) \in \Gamma_{j}$, each $y \in Z_{j}$ with $\operatorname{dist}(x, y) \leq \delta$, and each non-negative integer $i$, we have

$$
\left|(v(\ell) \cdot \nabla)^{i} P_{j}(y)\right| \lesssim_{D, i}|\log \delta|^{i} \delta
$$

Proof. First, we can assume without loss of generality that the largest coefficient of $P$ has magnitude 1. If $Z(P) \cap B(0,1)=\emptyset$ then $Z=\emptyset$ and thus $\Sigma=\emptyset$ so the result is trivial. Thus we can also assume that at least one non-constant coefficient of $P$ has magnitude $\gtrsim_{D} 1$.

For each point $z \in B(0,1)$, define

$$
m(z)=\sup _{x \in B(z, \delta)}|\nabla P(x)|
$$

Observe that $|\nabla P(x)|^{2}$ is a polynomial, all of whose coefficients have magnitude $\lesssim_{D} 1$ and at least one coefficient has magnitude $\gtrsim_{D} 1$. Thus

$$
\delta^{O_{D}(1)} \lesssim_{D} m(z) \lesssim_{D} 1 \text { for all } z \in B(0,1) .
$$

For each $z \in N_{\delta}(Z) \cap B(0,1)$, we have

$$
P(B(z, \delta)) \subset[-\delta m(z), \delta m(z)]
$$

By Lemma 2.5, there is a constant $c \gtrsim_{D} 1$ so that for each $z \in B(0,1)$,

$$
|\{x \in B(z, \delta)\}:| \nabla P(x) \mid \geq c m(z)\}\left|\geq \frac{99}{100}\right| B(x, \delta) \mid .
$$

This implies that $P(B(z, \delta))$ contains an interval of length $\gtrsim_{D} \delta m(z)$. Let $m_{1}, \ldots, m_{b}$, $b \lesssim_{D}|\log \delta|$ be a geometric sequence of non-negative numbers with $m_{1}=\delta^{O_{D}(1)}, m_{b}=$ $O_{D}(1)$ and $m_{j+1}=2 m_{j}$. If we select $m_{1}$ and $b$ appropriately, then for each $z \in$ $N_{\delta}(Z) \cap B(0,1)$ there exists an index $j$ so that $m_{j} \leq m(z)<m_{j+1}$. For each index 
$j$, let $w_{j, 1}, \ldots, w_{j, h}, h=O_{D}(1)$ be real numbers in $[-\delta m(z), \delta m(z)]$ so that for every $z \in N_{\delta}(Z) \cap B(0,1)$ satisfying $m_{j} \leq m(z)<m_{j+1}$, we have

$$
\left\{w_{j, 1}, \ldots, w_{j, h}\right\} \cap P(B(z, \delta)) \neq \emptyset
$$

i.e. at least one of the values $w_{j, 1}, \ldots, w_{j, h}$ is contained in $P(B(z, \delta))$. This can always be achieved since $P(B(z, \delta))$ must contain an interval of length $\gtrsim_{D} \delta m(z)$. We can select $h=O_{D}(1)$ to be independent of $j$. Observe that there are $O_{D}(|\log \delta|)$ pairs of indices $j, h$, which establishes Item 1 in the statement of the lemma (later in the proof we will re-index the pairs $(j, h)$ to use a single indexing variable).

For each $j=1, \ldots, b$ and each $k=1, \ldots, h$, define

$$
X_{j, k}=\left\{z \in N_{\delta}(Z) \cap B(0,1): m_{j} \leq m(z)<m_{j+1}, \text { and } w_{j, k} \in P(B(z, \delta))\right\} .
$$

Then each set $X_{j, k}$ is semi-algebraic of complexity $O_{D}(1)$, and

$$
N_{\delta}(Z) \cap B(0,1)=\bigcup_{j=1}^{b} \bigcup_{k=1}^{h} X_{j, k}
$$

For each $\ell \in \Sigma$, there exists indices $j, k$ so that $\left|\ell \cap X_{j, k}\right| \geq c_{1} /|\log \delta|$, where $c_{1} \gtrsim_{D} 1$. Since the set $\ell \cap X_{j, k}$ is semi-algebraic of complexity $O_{D}(1)$, it contains an interval of length $\geq c_{2} /|\log \delta|$, where $c_{2} \gtrsim_{D} 1$. Define

$$
\Sigma_{j, k}=\left\{\ell \in \Sigma: \ell \cap X_{j, k} \text { contains an interval of length } \geq c_{2} /|\log \delta|\right\} .
$$

With this definition, each set $\Sigma_{j, k}$ is semi-algebraic of complexity $O_{D, E}(1)$ and $\Sigma=$ $\bigcup \Sigma_{j, k}$, so Item 4 in the statement of the lemma is satisfied.

Define

$\Gamma_{j, k}=\left\{(x, \ell): \ell \in \Sigma_{j, k}, x\right.$ is contained in an interval in $\ell \cap X_{j, k}$ of length $\left.\geq c_{2} /|\log \delta|\right\}$.

Then $\left|\Gamma_{j, k}(\ell)\right|{ }_{D}|\log \delta|^{-1}$ for each $\ell \in \Sigma_{j, k}$, so Item 5 is satisfied. Define

$$
P_{j, k}(z)=m_{j}^{-1}\left(P(z)-h_{j, k}\right)
$$

each polynomial $P_{j, k}(z)$ has degree $\leq D$, so Item 2 is satisfied. If $(x, \ell) \in \Gamma_{j, k}$, then

$$
\left|P_{j, k}(y)\right| \leq 4 \delta \quad \text { for all } y \in B(x, \delta) .
$$

This is because $m(x)<2 m_{j}$, so $\left|\nabla P_{j, k}(y)\right| \leq 2$ for all $y \in B(x, \delta)$. (13) then follows from the fact that $B(x, \delta) \cap N_{2 \delta}\left(Z\left(P_{j, k}\right)\right) \neq \emptyset$. 
We have that if $(x, \ell) \in \Gamma_{j, k}$, then $x \in N_{\delta}\left(Z_{j, k}\right)$, where

$$
Z_{j, k}=\left\{z \in Z\left(P_{j, k}\right) \cap B(0,1): 1 \leq\left|\nabla P_{j, k}(z)\right| \leq 2\right\}
$$

Thus Item 3 is satisfied.

It remains to verify Item 6 . Fix indices $j, k$ and let $\Gamma=\Gamma_{j, k}$. Let $(x, \ell) \in \Gamma$ and let $y \in Z_{j, k}$ with $\operatorname{dist}(x, y) \leq \delta$. Then there is a line segment $I \subset \Gamma(\ell)$ of length $\gtrsim_{D}|\log \delta|^{-1}$ containing $x$. By (13) we have that $\left|P_{j, k}(z)\right| \leq 4 \delta$ for all $z \in N_{\delta}(I)$. Let

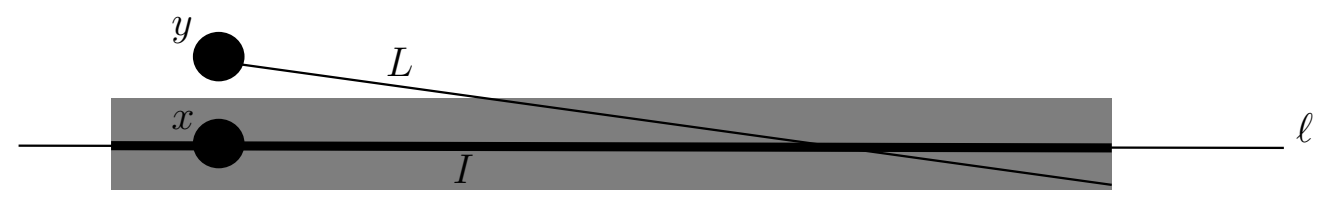

Figure 1: The points $x$ and $y$ (black circles); the lines $\ell$ and $L$ (thin black lines); the line segment $I$ (thick black line) and the region $N_{\delta}(I)$ (grey rectangle). Observe that if $\operatorname{dist}(x, y) \leq \delta$, then $\left|L \cap N_{\delta}(I)\right| \gtrsim|I|$

$L$ be a line containing $y$ with $\left|L \cap N_{\delta}(I)\right| \gtrsim_{D}|\log \delta|^{-1}$ (see Figure 1). Let $L(t): \mathbb{R} \rightarrow L$ be a unit speed parameterization of $L$, with $L(0)=y$ (i.e. $L(t)=y+t v(L)$ ). Then $P_{j, k}(L(t))$ is a univariate polynomial of degree $\leq D$ that satisfies $\left|P_{j, k}(L(t))\right| \leq 4 \delta$ for all $t$ in an interval $J \subset[0,1]$ of length $\gtrsim_{D}|\log \delta|^{-1}$. This implies that

$$
\left.\left|\frac{d^{i}}{d t^{i}} P_{j, k}(L(t))\right|_{t=0}\left|\lesssim_{D, i}\right| \log \delta\right|^{-i} \delta
$$

which gives us $10 p$.

The sets $\left\{\Sigma_{j, k}\right\}$ and $\left\{\Gamma_{j, k}\right\}$, and the polynomials $\left\{P_{j, k}\right\}$ satisfy the conclusions of Lemma 3.1. All that remains is to re-index the indices $j, k$ to use a single indexing variable.

\section{Curved varieties and the second fundamental form}

In this section, we will consider the region where $Z$ has small second fundamental form. We will show that lines lying near this region must be contained in a thin neighborhood of a hyperplane; this will be the set of lines $\Sigma_{3}$ from the statement of Theorem 1.2. This result will be proved in Proposition 4.1, which is the main result of this section. 


\subsection{A primer on the second fundamental form}

Define the functions $\varphi_{i}: \mathbb{R}^{4} \rightarrow \mathbb{R}^{4}, i=0,1,2,3$ by

$$
\begin{aligned}
& \varphi_{0}\left(x_{1}, x_{2}, x_{3}, x_{4}\right)=\left(\begin{array}{cccc}
x_{1}, & x_{2}, \quad x_{3}, & x_{4}
\end{array}\right), \\
& \varphi_{1}\left(x_{1}, x_{2}, x_{3}, x_{4}\right)=\left(-x_{2}, \quad x_{1},-x_{4}, \quad x_{3}\right) \text {, } \\
& \varphi_{2}\left(x_{1}, x_{2}, x_{3}, x_{4}\right)=\left(-x_{3}, \quad x_{4}, \quad x_{1},-x_{2}\right) \text {, } \\
& \varphi_{3}\left(x_{1}, x_{2}, x_{3}, x_{4}\right)=\left(-x_{4},-x_{3}, \quad x_{2}, \quad x_{1}\right) \text {. }
\end{aligned}
$$

Note that for each $x \in \mathbb{R}^{4}, \varphi_{0}(x), \varphi_{1}(x), \varphi_{2}(x)$, and $\varphi_{3}(x)$ have the same magnitude and are orthogonal.

Let $P \in \mathbb{R}\left[x_{1}, \ldots, x_{4}\right]$ and let $x \in Z(P)$. Suppose that $\nabla P(x) \neq 0$ and that $Z(P)$ is a smooth manifold in a neighborhood of $x$. Define $N(x)=\frac{\nabla P(x)}{|\nabla P(x)|}$. For each $i, j \in\{1,2,3\}$, define

$$
a_{i j}(x)=\left.\left(\varphi_{i}(\nabla P(x)) \cdot \nabla_{y}\right)\left(\varphi_{j}(\nabla P(x)) \cdot \nabla_{y}\right) P(y)\right|_{y=x} .
$$

To untangle the above definition: $\nabla P(x)$ is a vector in $\mathbb{R}^{4} ; \varphi_{i}(\nabla P(x))$ is a vector in $\mathbb{R}^{4} ;\left(\varphi_{i}(\nabla P(x)) \cdot \nabla_{y}\right)$ is an operator acting on functions $f: \mathbb{R}^{4} \rightarrow \mathbb{R}$. Similarly, $\left(\varphi_{j}(\nabla P(x)) \cdot \nabla_{y}\right)$ is an operator acting on functions $f: \mathbb{R}^{4} \rightarrow \mathbb{R}$. We apply these operators to the function $P(y)$, and then evaluate the resulting function at the point $y=x$.

Note that for each $i, j \in\{1,2,3\}, a_{i j} \in \mathbb{R}\left[x_{1}, \ldots, x_{4}\right]$ is a polynomial of degree $O(\operatorname{deg} P)$. Define

$$
I I(x)=\frac{1}{|\nabla P(x)|^{3}}\left[\begin{array}{lll}
a_{11}(x) & a_{12}(x) & a_{13}(x) \\
a_{21}(x) & a_{22}(x) & a_{23}(x) \\
a_{31}(x) & a_{32}(x) & a_{33}(x)
\end{array}\right]
$$

Then $I I(x)$ is the second fundamental form of $Z(P)$ at $x$, written in the basis $\frac{\varphi_{1}(x)}{\left|\varphi_{1}(x)\right|}, \frac{\varphi_{2}(x)}{\left|\varphi_{2}(x)\right|}, \frac{\varphi_{3}(x)}{\left|\varphi_{3}(x)\right|}$ (this is a basis for the tangent space $T_{x}(Z(P))$ ). Note that if the polynomial $P(x)$ is replaced by $t P(x)$ for $t \neq 0$, then the matrix $I I(x)$ is unchanged. For each $x \in \mathbb{R}^{4}$, define $\|I I(x)\|_{\infty}$ to be the $\ell^{\infty}$ norm of the entries of $I I(x)$ (so $\|I I(x)\|_{\infty}$ is a function from $\mathbb{R}^{4}$ to $\mathbb{R}$ ). Observe that if $P \in \mathbb{R}\left[x_{1}, \ldots, x_{4}\right]$ is a polynomial of degree at most $D$, then for each $\kappa>0$, the set

$$
\left\{x \in Z(P):\|I I(x)\|_{\infty}>\kappa\right\}
$$

is semi-algebraic of complexity $O_{D}(1)$. 
Note that if $0 \in Z(P)$ and if $N(0)=(0,0,0,1)$, then in a neighborhood of the origin we can write $Z(P)$ as the graph $x_{4}=f\left(x_{1}, x_{2}, x_{3}\right)$, with $f(0,0,0)=0$ and $\nabla f(0)=(0,0,0)$. Then

$$
I I(0)=\left[\begin{array}{lll}
\partial_{x_{1} x_{1}} f(0) & \partial_{x_{1} x_{2}} f(0) & \partial_{x_{1} x_{3}} f(0) \\
\partial_{x_{2} x_{1}} f(0) & \partial_{x_{2} x_{2}} f(0) & \partial_{x_{2} x_{3}} f(0) \\
\partial_{x_{3} x_{1}} f(0) & \partial_{x_{3} x_{2}} f(0) & \partial_{x_{3} x_{3}} f(0)
\end{array}\right] .
$$

Lemma 4.1. Let $P \in \mathbb{R}\left[x_{1}, \ldots, x_{4}\right]$ and let $M \subset Z(P)$ be a smooth manifold. Suppose that $\nabla P(x) \neq 0$ for all $x \in M,\|I I(x)\|_{\infty} \leq \kappa$ for all $x \in M$, and that every pair of points in $M$ can be connected by a smooth curve of arclength $\leq t$. Then the image of the Gauss map $N(M)$ can be contained in a ball of diameter $t \kappa$.

Proof. Fix a point $x_{0} \in M$. For each $x \in M$, let $\gamma(s)$ be a unit-speed paramaterization of a smooth curve with $\gamma(0)=x_{0}$ and $\gamma\left(s_{0}\right)=x$. By hypothesis, we can select such a curve with $s_{0} \leq t$. Then $\left|\frac{d}{d s} N(\gamma(s))\right| \leq\|I I(\gamma(s))\|_{\infty} \leq \kappa$; here $N(\gamma(s))$ is a map from $\mathbb{R}$ to the unit sphere $S^{3} \subset \mathbb{R}^{4}$, and $|\cdot|$ denotes the Euclidean norm of the four-dimensional vector $\frac{d}{d s} N(\gamma(s))$. We conclude that $N(x)$ is contained in the ball (in $S^{3}$ ) centered at $x_{0}$ of radius $t \kappa$.

Lemma 4.2 (Hypersurfaces with small second fundamental form lie near a hyperplane). Let $S \subset \mathbb{R}^{4}$ be a semi-algebraic set contained in $B(0,1)$ of complexity at most $E$. Suppose that $S$ is a connected smooth three-dimensional manifold and that $\|\mathrm{II}(x)\|_{\infty} \leq \kappa$ for all $x \in S$. Then $S$ can be contained in the $O_{E}(\kappa)$-neighborhood of a hyperplane.

Proof. After applying a rigid transformation, we can assume that $0 \in S$ and $N(0)=$ $(0,0,0,1)$. Let $x \in S$. By Lemma 2.4, we can find a smooth curve $\gamma \subset S$ of length $O_{E}(1)$ whose endpoints are 0 and $x$. Let $\gamma(s)$ be a unit-speed parameterization of this curve, so $\gamma(0)=0$ and $\gamma\left(s_{0}\right)=x$, for some $s_{0}=O_{E}(1)$.

Since $\frac{d}{d s} \gamma(s) \in T_{\gamma(s)} S$, we must have $\left|\frac{d}{d s} \gamma(s) \cdot(0,0,0,1)\right| \lesssim s_{0} \kappa$. In particular, the $x_{4}$ coordinate of $\gamma(s)$ must have magnitude $\lesssim s_{0} s \kappa=O_{E}(\kappa)$. Thus after applying a rigid transformation, $S$ is contained in the $O_{E}(\kappa)$-neighborhood of the hyperplane $\left\{x_{4}=0\right\}$.

Lemma 4.3 (Hypersurfaces with large second fundamental form escape every hyperplane). Let $P \in \mathbb{R}\left[x_{1}, \ldots, x_{4}\right]$ be a polynomial of degree at most $D$ and let $Z \subset$ $Z(P) \cap B(0,1)$ be a semi-algebraic set of complexity at most E. Suppose that $1 \leq$ $|\nabla P(x)| \leq 2$ and $\|I I(x)\|_{\infty} \geq \kappa$ at every point $x \in Z$. Then for every hyperplane $H$ and every $0<a \leq b \leq 1$, we have

$$
\left|N_{a}(Z) \cap N_{b}(H)\right| \lesssim_{D, E} a(b / \kappa)^{1 / 2}
$$


Proof. Let $\rho{ }_{D} 1$ be a small parameter to be determined later. Partition $Z$ into $O_{\rho, D, E}(1)=O_{D, E}(1)$ connected semi-algebraic sets so that on each set $Z^{\prime}$, each of $\partial_{x_{i}} P(x), i \in\{1,2,3,4\}$ vary by at most an additive factor of $\rho$. It suffices to establish (14) for each of these sets individually. Fix one of these sets $Z^{\prime}$. After applying a rotation, we can assume that for all $x \in Z^{\prime}$ we have $1-\rho \leq\left|\partial_{x_{4}} P(x)\right| \leq 2+\rho$ and $\left|\partial_{x_{i}} P(x)\right| \leq \rho, i=1,2,3$. In particular, each of the vectors $\varphi_{i}(N(x)), i=0,1,2,3$ are constant up to an additive factor of $\rho$ for all $x \in Z^{\prime}$.

Since $\|I I(x)\|_{\infty} \geq \kappa$ for all $x \in Z^{\prime}$, and $1 \leq|\nabla P(x)| \leq 2$, there is a unit vector $v \in T_{x}\left(Z^{\prime}\right)$ with $\left|(v \cdot \nabla)^{2} P(x)\right| \geq \kappa$. Phrased differently, there is a unit vector $\left(v_{1}, v_{2}, v_{3}\right) \in \mathbb{R}^{3}$ so that

$$
\left|\left(\left(v_{1} \varphi_{1}(N(x))+v_{2} \varphi_{2}(N(x))+v_{3} \varphi_{3}(N(x))\right) \cdot \nabla\right)^{2} P(x)\right| \geq \kappa .
$$

Since the map

$$
\left(v_{1}^{\prime}, v_{2}^{\prime}, v_{3}^{\prime}\right) \mapsto\left|\left(\left(v_{1}^{\prime} \varphi_{1}(N(x))+v_{2}^{\prime} \varphi_{2}(N(x))+v_{3}^{\prime} \varphi_{3}(N(x))\right) \cdot \nabla\right)^{2} P(x)\right|^{2}
$$

is homogeneous of degree four, there is a constant $c>0$ so that for all $v^{\prime}=\left(v_{1}^{\prime}, v_{2}^{\prime}, v_{3}^{\prime}\right)$ with $\angle\left(v, v^{\prime}\right) \leq c$, we have

$$
\left|\left(\left(v_{1}^{\prime} \varphi_{1}(N(x))+v_{2}^{\prime} \varphi_{2}(N(x))+v_{3}^{\prime} \varphi_{3}(N(x))\right) \cdot \nabla\right)^{2} P(x)\right| \geq \frac{99}{100} \kappa .
$$

Thus after further partitioning $Z^{\prime}$ into $O_{D}(1)$ connected semi-algebraic sets, we have that for each such set $Z^{\prime \prime}$, there is a unit vector $v$ so that

$$
\left|\left(\left(v_{1}^{\prime} \varphi_{1}(N(x))+v_{2}^{\prime} \varphi_{2}(N(x))+v_{3}^{\prime} \varphi_{3}(N(x))\right) \cdot \nabla\right)^{2} P(x)\right| \geq \frac{99}{100} \kappa
$$

for all $x \in Z^{\prime \prime}$ and all vectors $v^{\prime}=\left(v_{1}^{\prime}, v_{2}^{\prime}, v_{3}^{\prime}\right) \in S^{2} \subset \mathbb{R}^{3}$ with $\angle\left(v, v^{\prime}\right) \leq c$. It suffices to establish (14) for each of these sets $Z^{\prime \prime}$ individually. Fix such a set.

Fix a point $x_{0} \in Z^{\prime \prime}$ and apply a rigid transformation so that $N\left(x_{0}\right)=(0,0,0,1)$ and $v=(1,0,0)$. Note that $\varphi_{1}\left(N\left(x_{0}\right)\right)=(0,0,-1,0)$. If $\rho \gtrsim_{D} 1$ is selected sufficiently small, then $\angle\left(\varphi_{1}\left(N\left(x_{0}\right)\right), \varphi_{1}(N(x))\right) \leq c / 2$ for all $x \in Z^{\prime \prime}$. This means that for all $x \in Z^{\prime \prime}$, if $v^{\prime} \in \mathbb{R}^{4}$ is a unit vector in $T_{x}\left(Z^{\prime \prime}\right)$ with $\angle\left(v^{\prime},(0,0,-1,0)\right) \leq c / 2$, then $\left|\left(v^{\prime} \cdot \nabla\right)^{2} P(x)\right| \geq \frac{99}{100} \kappa$.

This means that we can write $Z^{\prime \prime}$ as the graph of a function $f\left(x_{1}, x_{2}, x_{3}\right)$; more precisely, for each $\left(x_{1}, x_{2}, x_{3}, x_{4}\right) \in Z^{\prime \prime}$, we can write $x_{4}=f\left(x_{1}, x_{2}, x_{3}\right)$, and

$$
\left|\partial_{x_{3}}^{2} f\left(x_{1}, x_{2}, x_{3}\right)\right| \gtrsim_{D} \kappa .
$$


Let $\pi\left(x_{1}, x_{2}, x_{3}, x_{4}\right)=\left(x_{1}, x_{2}, x_{3}\right)$, so $Z^{\prime \prime}$ is the graph of $f$ above $\pi\left(Z^{\prime \prime}\right)$. By (15), we have that if $I \subset \pi\left(Z^{\prime \prime}\right)$ is a line segment pointing in the direction $(0,0,1)$ and if $\left|f\left(x_{1}, x_{2}, x_{3}\right)\right| \leq 2 b$ for all $\left(x_{1}, x_{2}, x_{3}\right) \in I$, then we must have $|I| \lesssim_{D}(b / \kappa)^{1 / 2}$. Next, let $L$ be a line in $\mathbb{R}^{3}$ pointing in the direction $(0,0,1)$. Then

$$
\left|\left\{\left(x_{1}, x_{2}, x_{3}\right) \in L:\left|f\left(x_{1}, x_{2}, x_{3}\right)\right| \leq 2 b\right\}\right| \lesssim_{D}(b / \kappa)^{1 / 2},
$$

where $|\cdot|$ denotes one dimensional Lebesgue measure. Thus by Fubini's theorem,

$$
\left|\left\{\left(x_{1}, x_{2}, x_{3}\right) \in \pi\left(Z^{\prime \prime}\right):\left|f\left(x_{1}, x_{2}, x_{3}\right)\right| \leq 2 b\right\}\right| \lesssim_{D}(b / \kappa)^{1 / 2},
$$

where $|\cdot|$ denotes three dimensional Lebesgue measure. Thus

$$
\left|\left\{\left(x_{1}, x_{2}, x_{3}, x_{4}\right) \in Z^{\prime \prime}:\left|x_{4}\right| \leq 2 b\right\}\right| \lesssim_{D}(b / \kappa)^{1 / 2},
$$

where again $|\cdot|$ denotes three dimensional Lebesgue measure. We conclude that

$$
\left|N_{a}\left(Z^{\prime \prime}\right) \cap N_{b}(H)\right| \lesssim_{D, E} a\left|Z^{\prime \prime} \cap N_{a+b}(H)\right| \lesssim_{D, E} a(b / \kappa)^{1 / 2} .
$$

Since (16) holds for each of the $O_{D, E}(1)$ connected sets $Z^{\prime \prime}$, we obtain (14).

\subsection{Flat varieties are contained near a hyperplane}

Proposition 4.1. Let $0<\delta<\kappa$ and let $c>0$. Let $P \in \mathbb{R}\left[x_{1}, \ldots, x_{4}\right]$ be a polynomial of degree at most D. Define

$$
Z=\left\{x \in Z(P) \cap B(0,1): \nabla P(x) \neq 0,\|I I(x)\|_{\infty} \leq \kappa\right\} .
$$

Then there is a set of $O_{D}\left(c^{-O(1)}\right)$ rectangular prisms of dimensions $2 \times 2 \times 2 \times \kappa$ so that every line in $\Sigma_{\delta, c}(Z)$ is contained on one of these prisms.

Proof. Let $Z_{1}, \ldots, Z_{p}, p=O_{D}(1)$ be the connected components of $Z$ (without loss of generality, we can assume that each of these components is a smooth 3-dimensional manifold.) Each line $\ell \in \Sigma_{\delta, c}(Z)$ satisfies $\left|\ell \cap N_{\delta}\left(Z_{j}\right)\right| \geq c / p$ for some index $j$. By Lemma 4.2. each connected component $Z_{j}$ can be contained in the $\kappa^{\prime}=O_{D}(\kappa)-$ neighborhood of a hyperplane; call this hyperplane $H_{j}$.

Finally, for each index $j$, we can select $O_{D}\left(c^{-O(1)}\right)$ rectangular prisms of dimensions $2 \times 2 \times 2 \times \kappa$ so that every line $\ell$ satisfying $\left|\ell \cap N_{\kappa^{\prime}}\left(H_{j}\right)\right| \geq c / p$ must must be covered by one of these prisms. 


\section{$5 \quad$ Multilinearity and quantitative broadness}

In this section we will explore the notion of "broadness," which was introduced by Guth in [10] to study the restriction problem. Throughout this section, we will often refer to the following "standard setup."

Definition 5.1 (Standard setup). Let $d$ be a positive integer. Let $Z \subset \mathbb{R}^{d}$ and let $\Phi \subset Z \times S^{d-1}$ be semi-algebraic sets of complexity at most $E$. Let $\pi_{Z}: \Phi \rightarrow Z$ and $\pi_{S}: \Phi \rightarrow S^{d-1}$ be the projection of $\Phi$ to $Z$ and $S^{d-1}$, respectively. For each $z \in Z$, define $\Phi(z)=\pi_{Z}^{-1}(z)$.

\section{$5.1 \quad(m, A)$-broadness}

Definition 5.2. Let $d, \Phi$, and $Z$ be defined as in the standard setup from Definition 5.1. For each positive integer $0 \leq m \leq d-1$, let $\mathcal{S}_{m}$ be the set of $m$ dimensional unit spheres contained in $S^{d-1}$ (recall that a zero dimensional unit sphere in $S^{d-1}$ is just a pair of antipodal points). We will identify $\mathcal{S}_{m}$ with a semi-algebraic subset of $\mathbb{R}^{N}$ for some $N=O_{d}(1)$. Let $A \geq 1$ be an integer and let $u \geq 0$. Define

$$
\begin{array}{r}
(m, A)-\operatorname{Narrow}_{u}(\Phi)=\left\{z \in Z: \exists S_{1}, \ldots, S_{A} \in \mathcal{S}_{m} \text { s.t. } \forall v \in \pi_{S}(\Phi(z))\right. \\
\left.\exists i \in\{1, \ldots, A\} \text { s.t. } \angle\left(v, S_{i}\right\} \leq u\right\} .
\end{array}
$$

In words, $z \in(m, A)-\operatorname{Narrow}_{u}(\Phi)$ if and only if there is a set of $A m$-dimensional unit spheres $S_{1}, \ldots, S_{A}$ so that every vector $v \in \pi_{S}(\Phi(z))$ makes an angle at most $u$ with one of these spheres. Observe that if $m \leq m^{\prime}, A \leq A^{\prime}$, and $u \leq u^{\prime}$, then $(m, A)-\operatorname{Narrow}_{u}(\Phi) \subset\left(m^{\prime}, A^{\prime}\right)-\operatorname{Narrow}_{u^{\prime}}(\Phi)$.

Define

$$
(m, A)-\operatorname{Broad}_{u}(\Phi)=Z \backslash(m, A)-\operatorname{Narrow}_{u}(\Phi) .
$$

If $(m, A)-\operatorname{Narrow}_{u}(\Phi)=Z$, we say that $\Phi$ is $(m, A)$-narrow at width $u$. If $(m, A)-\operatorname{Broad}_{u}(\Phi)=$ $Z$, we say that $\Phi$ is $(m, A)$-broad at width $u$.

The sets $(m, A)-\operatorname{Broad}_{u}(\Phi)$ and $(m, A)-\operatorname{Narrow}_{u}(\Phi)$ have complexity $O_{d, E}(1)$. In practice we will have $d=4$ so the complexity is $O_{E}(1)$.

Lemma 5.1. Let $d, \Phi$, and $Z$ be defined as in the standard setup from Definition 5.1. Let $u \leq s$. Let $m \geq 1$. Suppose that $\Phi$ is $(m, 1)$-narrow at width $u$ and $(m-1,1)$-broad at width $s$. Define

$$
A=\left\{(z, S) \in Z \times \mathcal{S}_{m}: \angle(v, S) \leq u \forall v \in \pi_{S}(\Phi(z))\right\}
$$


If we identify $Z \times \mathcal{S}_{m}$ with a subset of $\mathbb{R}^{d} \times \mathbb{R}^{N}, N=O_{d}(1)$, then

$$
\mathcal{E}_{u}(A) \lesssim_{d, E} u^{-\operatorname{dim}(Z)} s^{-\operatorname{codim}(Z)},
$$

where $\operatorname{codim}(Z)=d+N-\operatorname{dim}(Z)=O_{d}(1)$.

Proof. Since the constants $d$ and $E$ are fixed, all implicit constants may depend on these quantities. First, since $\Phi$ is $(m, 1)$-narrow at width $u$, i.e. $(m, 1)-\operatorname{Narrow}_{u}(\Phi)=$ $Z$, we have that $\pi: A \rightarrow Z$ is onto. Apply Lemma 2.6 to select a semi-algebraic set $A^{\prime} \subset A$ of complexity $O(1)$ so that $\pi: A^{\prime} \rightarrow Z$ is a bijection. In particular, we have $\operatorname{dim}\left(A^{\prime}\right)=\operatorname{dim}(Z)$.

We claim that if $(z, S), \quad\left(z, S^{\prime}\right) \in A$, then $\operatorname{dist}\left(S, S^{\prime}\right) \lesssim u / s$, where $\operatorname{dist}\left(S, S^{\prime}\right)$ denotes the Euclidean distance in $\mathbb{R}^{N}$ between the points in $\mathbb{R}^{N}$ identified with $S$ and $S^{\prime}$. Indeed, note that

$$
\pi_{S}(\Phi(z)) \subset N_{u}(S) \cap N_{u}\left(S^{\prime}\right) .
$$

Since $z \in(m-1,1)-\operatorname{Broad}_{s}(\Phi)$ we have that $\pi_{S}(\Phi(z)) \subset N_{u}(S) \cap N_{u}\left(S^{\prime}\right)$ cannot be contained in the $s$-neighborhood of a $(m-1)$-dimensional unit sphere, and thus $N_{u}(S) \cap N_{u}\left(S^{\prime}\right)$ cannot be contained in the $s$-neighborhood of a $(m-1)$-dimensional unit sphere. This implies $\operatorname{dist}\left(S, S^{\prime}\right) \lesssim u / s$.

By Lemma 2.3,

$$
\begin{aligned}
\mathcal{E}_{u}(A) & \lesssim \mathcal{E}_{u}\left(N_{u / s}\left(A^{\prime}\right)\right) \\
& \lesssim u^{\operatorname{dim}\left(A^{\prime}\right)} s^{-\operatorname{codim}\left(A^{\prime}\right)} \\
& =u^{\operatorname{dim}(Z)} s^{-\operatorname{codim}(Z)} .
\end{aligned}
$$

In practice, we will use Lemma 5.1 in the special case $Z \subset \mathbb{R}^{4}, \Phi \subset Z \times S^{3} \subset \mathbb{R}^{8}$, and $m=2$. The lemma will help us analyze the situation where a hypersurface $Z(P) \subset \mathbb{R}^{4}$ is ruled by planes.

\section{$5.2 \quad$ Strong broadness}

If $\mathcal{V} \subset S^{d-1}$ is semi-algebraic of complexity at most $E$, then by Lemma 2.1 there is a number $K_{d, E}$ so that $\mathcal{V}$ is a union of at most $K_{d, E}$ connected components. This means that for each $s>0$, either $\mathcal{V}$ can be contained in the $s$-neighborhood of a union of $K_{d, E}$ vectors, or $\mathcal{V}$ contains a connected component of diameter at least $s$.

Definition 5.3. Let $d, \Phi$, and $Z$ be defined as in the standard setup from Definition 5.1. Let $E_{1}=O_{d, E}$ be an integer so that $\pi_{S}(\Phi(z))$ has complexity at most $E_{1}$ for each $z \in Z$. 
Define

$$
1-\operatorname{SBroad}_{s}(\Phi)=\left(1, K_{d, E_{1}}\right)-\operatorname{Broad}_{s}(\Phi) .
$$

The "S" stands for "Strong." If $1-\operatorname{SBroad}_{s}(\Phi)=Z$, we say that $\Phi$ is strongly 1-broad at width $s$.

Note that if $z \in 1-\operatorname{SBroad}_{s}(\Phi)$, then $\pi_{S}(\Phi(z))$ contains a connected component of diameter $\geq s$. Conversely, if $\pi_{S}(\Phi(z))$ contains a connected component of diameter at least $K_{d, E_{1}} s$, then $z \in 1-\operatorname{SBroad}_{s}(\Phi)$. It would be preferable to just directly define $1-\operatorname{SBroad}_{s}(\Phi)$ to be the set of points $z \in Z$ so that $\pi_{S}(\Phi(z))$ contains a connected component of diameter at least $s$, but it is not clear whether $1-\operatorname{SBroad}_{s}(\Phi)$ would be semi-algebraic with this definition. Under Definition $5.3,1-\operatorname{SBroad}_{s}(\Phi) \subset Z$ is semi-algebraic of complexity $O_{d, E}(1)$.

Remark 5.1. It would be straightforward to define a notion of strong $m$-broadness for each $m \geq 1$, but this definition is not particularly useful if $m>1$. One of the key properties of strong 1-broadness is that if $z \in 1-\operatorname{SBroad}_{s}(\Phi)$, then

$$
\mathcal{E}_{s}\left(\pi_{S}(\Phi(z))\right) d_{d, E} s^{-1}
$$

Unfortunately, the analogous statement for strong $m$-broadness (with $s^{-1}$ replaced by $s^{-m}$ ) need not be true.

Lemma 5.2. Let $d, \Phi$, and $Z$ be defined as in the standard setup from Definition 5.1 and let $s>0$. Suppose that $1-\operatorname{SBroad}_{s}(\Phi)=\emptyset$. Then

$$
\mathcal{E}_{s}(\Phi) \lesssim_{d, E} s^{-\operatorname{dim}(Z)}
$$

Proof. Since $d$ and $E$ are fixed, all implicit constants may depend on these quantities. For each $k=1, \ldots, O(1)$, define

$$
A_{k}=\left\{\left(z, v_{1}, \ldots, v_{k}\right): z \in Z, v_{1}, \ldots, v_{k} \in \pi_{S}(\Phi(z)), \angle\left(v_{i}, v_{j}\right) \geq s \text { if } i \neq j\right\} .
$$

Let $\pi_{k}: A_{k} \rightarrow Z$ be the projection to $Z$, and let $Z_{k}=\pi_{k}\left(A_{k}\right)$. Since $1-\operatorname{SBroad}_{s}(\Phi)=$ $\emptyset$, we have that

$$
\bigcup_{k=1}^{O(1)} Z_{k}=\pi_{Z}(\Phi) .
$$

Apply Lemma 2.6 to each map $\pi_{k}: A_{k} \rightarrow Z_{k}$ to obtain sets $A_{k}^{\prime} \subset A_{k}$ so that the projection map $\pi_{k}: A_{k}^{\prime} \rightarrow Z_{k}$ is a bijection. Define $Z_{k}^{\prime}=Z_{k} \backslash \bigcup_{j>k} Z_{j}$. Note that each set $Z_{k}^{\prime}$ has dimension $\leq \operatorname{dim}(Z)$ and is semi-algebraic of complexity $O(1)$. 
Define

$$
\Phi_{k}=\left\{(z, v) \in \Phi: z \in Z_{k}^{\prime}\right\}
$$

Then by 18 , we have

$$
\Phi=\bigsqcup_{k=1}^{O(1)} \Phi_{k} .
$$

Note that if $z \in Z_{k}^{\prime}$ and if $\left(z, v_{1}, \ldots, v_{k}\right)=\pi_{k}^{-1}(z)$ (here $\pi_{k}: A_{k}^{\prime} \rightarrow Z_{k}^{\prime}$ is a bijection, so it has a well-defined inverse), then every vector in $\pi_{S}(\Phi(z))$ must be close to one of the vectors $v_{1}, \ldots, v_{k}$; more precisely,

$$
\pi_{S}(\Phi(z)) \subset N_{s}\left(\left\{v_{1}, \ldots, v_{k}\right\}\right) .
$$

Indeed, if 20$]$ did not hold, then there exists $v_{k+1} \in \pi_{S}(\Phi(z)) \backslash N_{s}\left(\left\{v_{1}, \ldots, v_{k}\right\}\right)$. But then $\left(z, v_{1}, \ldots, v_{k}, v_{k+1}\right) \in A_{k+1}$, so $z \in Z_{k+1}$, which contradicts the assumption that $z \in Z_{k}^{\prime}$.

For each index $k$ and each $j=1, \ldots, k$, define the projections $\pi_{k, j}: A_{k}^{\prime} \rightarrow Z_{k}^{\prime} \times$ $S^{d-1}$ by $\left(z, v_{1}, \ldots, v_{k}\right) \mapsto\left(z, v_{j}\right)$. Define $W_{k}=\bigcup_{j=1}^{k} \pi_{k, j}\left(A_{k}^{\prime}\right)$. Thus $W_{k} \subset Z_{k}^{\prime} \times S^{d-1}$, and for each $z \in Z_{k}^{\prime}$, we have that $W_{k} \cap\left(\{z\} \times S^{d-1}\right)$ is the set $\left\{\left(z, v_{1}\right), \ldots,\left(z, v_{k}\right)\right\}$, where $\left(z, v_{1}, \ldots, v_{k}\right)=\pi_{k}^{-1}(z)$.

Observe that $W_{k}$ is a semi-algebraic set of dimension $\leq \operatorname{dim}(Z)$ and complexity $O(1)$, and thus by Lemma 2.3 .

$$
\mathcal{E}_{s}\left(W_{k}\right) \lesssim s^{-\operatorname{dim}(Z)}
$$

On the other hand, by $(20)$ we have that

$$
\mathcal{E}_{s}\left(\Phi_{k}\right) \lesssim \mathcal{E}_{s}\left(W_{k}\right)
$$

The lemma now follows from (19), 20), 21), and (22).

\subsection{Bundles of lines and the standard setup}

In this section we will relate the objects $Z, \Sigma$ and $\Gamma$ from Section 2.1 with the standard setup from Definition 5.1 .

Definition 5.4. Let $\delta>0$ and let $P \in \mathbb{R}\left[x_{1}, \ldots, x_{4}\right]$ be a polynomial of degree at most $D$. Let $Z \subset Z(P) \cap B(0,1)$ and $\Gamma \subset \Gamma\left(N_{\delta}(Z), \mathcal{L}\right)$ be semi-algebraic sets of complexity at most $E$. For each $x \in N_{\delta}(Z)$, define $f_{Z}(x)$ to be the point $z \in Z$ that minimizes $\operatorname{dist}(x, z)$. If more than one such point exists, select the one that is minimal under the lexicographic order (any semi-algebraic total order would be 
equally good). Then the set $\left\{(x, z) \in N_{\delta}(Z) \times Z: z=f_{Z}(x)\right\}$ is semi-algebraic of complexity $O_{E, D}(1)$. Define the set $\Phi \subset Z \times S^{3}$ to be the set of ordered pairs

$$
\Phi=\left\{\left(f_{Z}(x), v(\ell)\right):(x, \ell) \in \Gamma\right\} .
$$

Then $\Phi \subset Z \times S^{3}$ is semi-algebraic of complexity $O_{D, E}(1)$. We will say that set $\Phi$ is associated to $\Gamma$ (the set $Z$ and the parameter $\delta$ will be obvious from context).

By construction, the map $\Gamma \rightarrow \Phi,(x, \ell) \mapsto\left(f_{Z}(x), v(\ell)\right)$ is onto. If $\Phi^{\prime} \subset \Phi$, we will define $\Gamma^{\prime}$ to be the pre-image of $\Phi^{\prime}$ under this map.

\section{Narrow varieties}

In this section, we will consider the region where $Z$ either fails to be robustly 1-broad, or fails to be $(2,2)$-broad and has large second fundamental form. We will show that the lines having large intersection with this region must be covered by a union of two and three dimensional prisms (i.e. prisms with either two or three "long" directions). These sets of lines will be the sets $\Sigma_{1}$ and $\Sigma_{2}$ from the statement of Theorem 1.2 . The main results of this section are Proposition 6.1, which describes what happens when $Z$ fails to be robustly 1-broad, and Proposition 6.2, which describes what happens when $Z$ has large second fundamental form and fails to be $(2,2)$-broad.

\subsection{1-Narrow varieties are ruled by lines}

Proposition 6.1. Let $0<\delta<s$ and $c>\delta$. Let $P \in \mathbb{R}\left[x_{1}, \ldots, x_{4}\right]$ be a polynomial of degree at most $D$; let $Z=Z(P) \cap B(0,1)$; let $\Sigma \subset \Sigma_{\delta, c}(Z)$ be a semi-algebraic set of complexity at most $E$. Let $\Gamma \subset \Gamma\left(N_{\delta}(Z), \Sigma\right)$ be a semi-algebraic set of complexity at most E. Let $\Phi \subset Z \times S^{3}$ be associated to $\Gamma$, in the sense of Definition 5.4. Suppose that

$$
\begin{aligned}
& 1-\operatorname{SBroad}_{s}(\Phi)=\emptyset \\
& |\Gamma(\ell)| \geq c \quad \text { for every } \ell \in \Sigma .
\end{aligned}
$$

Then there is a set of $O_{D, E}\left(c^{-1} s^{-2}\right)$ rectangular prisms of dimensions $2 \times s \times s \times s$, so that every line from $\Sigma$ is covered by one of the prisms.

Proof. By Lemma 5.2 .

$$
\mathcal{E}_{s}(\Phi) \lesssim_{D, E} s^{-3}
$$


Let $\mathcal{R}_{\text {max }}$ be a maximal set of essentially disjoint rectangular prisms of dimensions $2 \times s / 4 \times s / 4 \times s / 4$ that intersect $B(0,1)$. For each $R \in \mathcal{R}_{\max }$, define $v(R)$ to be the direction of the long axis of $R$, and define

$$
R^{*}=\left\{(x, v) \in \mathbb{R}^{4} \times S^{3}: x \in R, \angle(v, v(R)) \leq s / 4\right\} .
$$

Observe that every line intersecting $B(0,1)$ is covered by some rectangular prism from $\mathcal{R}_{\max }$, and that for each $C \geq 1$, the $C$-fold dilates of the sets $\left\{C R^{*}\right\}_{R \in \mathcal{R}_{\max }}$ are $O_{C}(1)$-overlapping.

Let $(x, \ell) \in \Gamma$ and let $\left(f_{Z}(x), v(\ell)\right)$ be the corresponding element of $\Phi$. Note that if $\ell \times\{v(\ell)\} \cap R^{*} \neq \emptyset$ then $\ell \cap R \neq \emptyset$ and $\angle(v(\ell), v(R)) \leq s / 4$, and thus $\ell$ is covered by the 4 -fold dilate of $R$. Furthermore, if this happens then $\Phi \cap R^{*} \neq \emptyset$. Thus to prove the lemma, it suffices to show that

$$
\left|\left\{R \in \mathcal{R}_{\max }: R^{*} \cap \Phi \neq \emptyset\right\}\right| \lesssim_{D, E} c^{-1} s^{-2} .
$$

Let $R \in \mathcal{R}_{\max }$ and suppose $R^{*} \cap \Phi \neq \emptyset$. We will show that

$$
\mathcal{E}_{s}\left(\Phi \cap 4 R^{*}\right) \geq c / s
$$

To see this, let $(z, v) \in R^{*} \cap \Phi$. Then there exists a point $(x, \ell) \in \Gamma$ with $\operatorname{dist}(x, z)<\delta$ and $v(\ell)=v$. Thus

$$
\angle(v(\ell), v(R)) \leq s / 4 \text {. }
$$

Thus $\ell \cap B(0,1) \subset \ell \cap 4 S$. Since $\ell \in \Sigma$, we have $\mathcal{E}_{s}(\Gamma(\ell)) \geq c / s$ and thus there exist $\geq c / s s$-separated points $x^{\prime} \in \Gamma(\ell)$ with $\left(x^{\prime}, v(\ell)\right) \in S^{*}$. Of course for each of these points $x^{\prime} \in \Gamma(\ell)$ there exists a point $z^{\prime} \in B\left(x^{\prime}, \delta\right)$ with $\left(z^{\prime}, v(\ell)\right) \in \Phi \cap R^{*}$, which establishes (27). Since the sets $\left\{4 R^{*}\right\}_{\mathcal{R}_{\max }}$ are $O(1)$ overlapping, by combining (25) and (27) we have 26).

\subsection{2-narrow varieties are ruled by planes}

Lemma 6.1. Let $\delta, u, s, \kappa, c, D, E$ be parameters with $0<\delta<u<s<c$ and $\delta<\kappa$. Then there exists a number $w{ }_{D, E}(u s \kappa c)^{O(1)}$ so that the following holds.

Let $P \in \mathbb{R}\left[x_{1}, \ldots, x_{4}\right]$ be a polynomial of degree at most $D$ and let

$$
Z \subset\left\{z \in Z(P) \cap B(0,1): 1 \leq|\nabla P(z)| \leq 2,\|I I(z)\|_{\infty} \geq \kappa\right\} .
$$

Let $\Sigma \subset \Sigma_{\delta, c}(Z)$, and let $\Gamma \subset \Gamma\left(N_{\delta}(Z), \Sigma\right)$. Suppose that $Z, \Sigma$, and $\Gamma$ are semialgebraic sets of complexity at most $E$. Let $\Phi$ be associated to $\Gamma$, in the sense of 
Definition 5.4. Suppose that

$$
\begin{aligned}
& Z \subset(2,1)-\operatorname{Narrow}_{w}(\Phi), \\
& |\Gamma(\ell)| \geq c \quad \text { for all } \ell \in \Sigma .
\end{aligned}
$$

Then we can write $\Sigma=\Sigma^{\prime} \cup \Sigma^{\prime \prime}$, where the lines in $\Sigma^{\prime}$ can be covered by $O_{D, E}\left(c^{-O(1)} s^{-2}\right)$ rectangular prisms of dimensions $2 \times s \times s \times s$, and the lines in $\Sigma^{\prime \prime}$ can be covered by $O_{D, E}\left((c s)^{-O(1)} u^{-1}\right)$ rectangular prisms of dimensions $2 \times 2 \times u \times u$.

Proof. Since $D$ and $E$ are fixed, whenever we write $A \lesssim B$, the implicit constant may depend on these quantities. Let

$$
\Phi_{0}=\left\{(z, v) \in \Phi: z \in 1-\operatorname{SBroad}_{s}(\Phi)\right\},
$$

and let $\Phi_{0}^{\prime}=\Phi \backslash \Phi_{0}$. Define $\Gamma_{0}$ to be the pre-image of $\Phi_{0}$ under the map $\Gamma \rightarrow \Phi$, and define $\Gamma_{0}^{\prime}=\Gamma \backslash \Gamma_{0}$. Note that $\Gamma_{0}^{\prime}$ is the pre-image of $\Phi_{0}^{\prime}$. $\Gamma_{0}^{\prime}$ is a set of complexity $E_{1}=O_{D, E}(1)$; in particular, the constant $E_{1}$ can be chosen to be independent of $s$ and $\delta$.

Let

$$
\begin{aligned}
& \Sigma_{0}=\left\{\ell \in \Sigma:\left|\Gamma_{0}(x)\right|>c / 2\right\}, \\
& \Sigma_{0}^{\prime}=\left\{\ell \in \Sigma:\left|\Gamma_{0}^{\prime}(x)\right|>c / 2\right\} .
\end{aligned}
$$

We will put the lines in $\Sigma_{0}^{\prime}$ into $\Sigma^{\prime}$; by Proposition 6.1, these lines can be covered by $O\left(s^{-2} c^{-O(1)}\right)$ rectangular prisms of dimensions $2 \times s \times s \times s$.

Define

$$
Z_{1}=\left\{z \in Z: \Phi_{0}(z) \neq \emptyset\right\} \subset 1-\operatorname{SBroad}_{s}(\Phi) .
$$

Let $w>(u s \kappa c)^{O(1)}$ be a number that will be determined below. Define

$$
A_{1}=\left\{(z, S) \in Z_{1} \times \mathcal{S}_{1}: \angle(v, S) \leq w \forall v \in \pi_{S}(\Phi(z))\right\} .
$$

Since $Z_{1} \subset 1-\operatorname{SBroad}_{s}(\Phi)$, we have that if $(z, S),\left(z, S^{\prime}\right) \in A_{1}$, then

$$
\angle\left(S, S^{\prime}\right) \leq 2 w / s .
$$

This is because there exists two vectors $v, v^{\prime} \in \pi_{S}(\Phi(z))$ with $\angle\left(v, v^{\prime}\right) \geq s$, and these vectors satisfy $\angle(v, S) \leq w ; \angle\left(v, S^{\prime}\right) \leq w ; \angle\left(v^{\prime}, S\right) \leq w$; and $\angle\left(v^{\prime}, S^{\prime}\right) \leq w$. Observe that $A_{1}$ obeys the hypotheses of Lemma 5.1 .

Define $\pi: A_{1} \rightarrow Z_{1}$ to be the projection $(z, S) \mapsto z$. Then by (29) $\pi\left(A_{1}\right)=Z_{1}$. Use Lemma 2.6 to select a set $A_{1}^{\prime} \subset A_{1}$ so that $\pi: A_{1}^{\prime} \rightarrow Z_{1}$ is a bijection. By (32), we have that

$$
A_{1} \subset N_{2 w / s}\left(A_{1}^{\prime}\right) .
$$


For each $z \in Z_{1}$, define $S(z)$ to be the (unique) great circle $S$ so that $(z, S) \in A_{1}^{\prime}$. The function $S(z)$ is semi-algebraic of complexity $O(1)$. If $(x, \ell) \in \Gamma_{0}$, then $f_{Z}(x) \in$ $Z_{1}$. Thus for each $\ell \in \Sigma$, the set

$$
S \circ f_{Z} \circ \Gamma_{0}(\ell)=\left\{S\left(f_{Z}(x)\right): x \in \Gamma_{0}(\ell)\right\} \subset \mathcal{S}_{1}
$$

is a union of $O(1)$ connected components in $\mathcal{S}_{1}$ (recall that $\mathcal{S}_{1}$ is the parameter space of one-dimensional great circles in $S^{3}$ ).

For each $S \in \mathcal{S}_{1}$, define $\operatorname{span}(S)$ to be the two-dimensional vector space in $\mathbb{R}^{4}$ that contains the great circle $S$, i.e. $\operatorname{span}(S)=\{r v: r \in \mathbb{R}, v \in S\}$. For each $z \in Z_{1}$, Define $\Pi(z)=z+\operatorname{Span}(S(z))$; this is an affine 2-plane containing $z$.

Since $S \circ f_{Z} \circ \Gamma_{0}(\ell)$ is a union of $O(1)$ connected components, heuristically, this means that either (A): $\left\{\Pi \circ f_{Z}(z): z \in \Gamma_{0}(\ell)\right\}$ can be covered by the thickened neighborhoods of a small number of planes containing $\ell$, or $(\mathrm{B})$ : the union of the planes in $\left\{\Pi \circ f_{Z}(z): z \in \Gamma_{0}(\ell)\right\}$ fill out a large fraction of $N_{\delta}\left(Z_{1}\right)$. We will make this heuristic precise in the arguments below.

let $h \gtrsim c$ be a constant that will be determined below. Define

$$
\begin{aligned}
& Y=\left\{(\ell, S, x) \in \Sigma_{0}\right. \times \mathcal{S}_{1} \times \mathbb{R}^{4}: x \in \Gamma_{0}(\ell), \\
&\left.\forall x^{\prime} \in \ell \cap B(x, h), \text { we have } x^{\prime} \in \Gamma_{0}(\ell) \text { and } \angle\left(S \circ f_{Z}\left(x^{\prime}\right), S\right)<u\right\} .
\end{aligned}
$$

Let $\pi_{\mathcal{L}}(\ell, S, x)=\ell$ and define $\Sigma_{1}=\pi_{\mathcal{L}}(Y), \Sigma_{2}=\Sigma_{0} \backslash \Sigma_{1}$. In words, $\Sigma_{1}$ is the set of lines $\ell \in \Sigma_{0}$ so that there exists a line segment $I \subset \Gamma_{0}(\ell)$ of length $2 h$ with the property that the great circle $S \circ f_{Z}\left(x^{\prime}\right)$ does not change much as $x^{\prime}$ moves along $I$. Remark 6.1. Heuristically, if the variety $Z(P)$ was ruled by planes, and if every line was contained in one of these planes, then $\Sigma_{1}=\Sigma_{0}$. We will show that if this is the case, then $\Sigma_{1}$ can be partitioned into disjoint pieces that do not interact with each other (geometrically, if $Z(P)$ is ruled by planes and if every line lies in one of these planes, then we can write $Z(P)$ as a disjoint union of planes and consider each of these planes individually).

On the other hand, $\Sigma_{2}$ is the set of lines $\ell \in \Sigma_{0}$ so that for every interval $I \subset \Gamma_{0}(\ell)$ of length $2 h, S \circ f_{Z}(I)$ has diameter $\geq u$. Since $S \circ f_{Z} \circ \Gamma_{0}(\ell)$ is a union of $O(1)$ connected components, if $h \gtrsim c$ is selected sufficiently small, then there must exist an interval $I \subset \Gamma_{0}(\ell)$ of length $2 h$ so that $S \circ f_{Z}(I)$ is connected.

Remark 6.2. Heuristically, if the variety $Z(P)$ is a small perturbation of a hyperplane, then it could be the case that $\Sigma_{2}=\Sigma_{0}$. We will show that if this is the case, then most of $Z(P)$ can be contained in a thin neighborhood of a hyperplane, and this will contradict the assumption that $\|I I(z)\|_{\infty}$ is large on $Z$. 


\section{Understanding lines in $\Sigma_{1}$}

Apply Lemma 2.6 to the surjection $\pi_{\mathcal{L}}: Y \rightarrow \Sigma_{1}$ to obtain a set $Y^{\prime} \subset Y$ so that $\pi_{\mathcal{L}}: Y^{\prime} \rightarrow \Sigma_{1}$ is a bijection. Define $S(\ell)$ to be the circle in $\mathcal{S}_{1}$ containing the vector $v(\ell)$ that makes the smallest angle with the circle $\pi_{\mathcal{S}_{1}} \circ \pi_{\mathcal{L}}^{-1}(\ell)$. Observe that if $\ell \in \Sigma_{1}$ and if $(\ell, S, x)=\pi_{\mathcal{L}}^{-1}(\ell)$, then $(x, v(\ell)) \in \Gamma_{0}$ and thus $\angle(v(\ell), S)<w \leq u$, so $\angle(S, S(\ell))<u$.

For each $\ell \in \Sigma_{1}$, define $\Pi(\ell)=\ell+\operatorname{span}(S(\ell))$; this is an affine plane containing $\ell$ that points in the directions spanned by $S(\ell)$.

Define

$$
\Gamma_{1}=\left\{(x, \ell) \in \Gamma_{0}: \angle(S(\ell), S(x))<2 u\right\} .
$$

Then for each $\ell \in \Sigma_{1}$, we have $\left|\Gamma_{1}(\ell)\right| \geq 2 h$. This is because $\ell \in \Sigma_{1}$ implies that there exists a point $(\ell, S, x)=\pi_{\mathcal{L}}^{-1}(x) \in Y$, and thus there exists an interval $I \subset \Gamma_{0}(\ell)$ of length $2 h$ containing $x$ so that for every point $x^{\prime} \in I$, we have

$$
\begin{aligned}
\angle\left(S(\ell), S\left(x^{\prime}\right)\right) & \leq \angle(S(\ell), S)+\angle\left(S\left(x^{\prime}\right), S\right) \\
& <u+u \\
& =2 u .
\end{aligned}
$$

Let $\Phi_{1}$ be the set associated to $\Gamma_{1}$, in the sense of Definition 5.4. Define

$$
\Phi_{2}=\left\{(z, v) \in \Phi_{1}: z \in 1-\operatorname{SBroad}_{s}\left(\Phi_{1}\right)\right\} .
$$

Define $\Gamma_{2} \subset \Gamma_{1}$ to be the pre-image of $\Phi_{2}$ and define $\Gamma_{2}^{\prime}=\Gamma_{1} \backslash \Gamma_{2}$ (this is the pre-image of $\left.\Phi_{2}^{\prime}=\Phi_{1} \backslash \Phi_{2}\right)$. Define

$$
\begin{aligned}
& \Sigma_{1}^{\prime}=\left\{\ell \in \Sigma_{1}:\left|\Gamma_{2}(\ell)\right| \geq h\right\}, \\
& \Sigma_{1}^{\prime \prime}=\left\{\ell \in \Sigma_{1}:\left|\Gamma_{2}^{\prime}(\ell)\right| \geq h\right\} .
\end{aligned}
$$

$\Gamma_{2}^{\prime}$ and $\Sigma_{1}^{\prime \prime}$ are semi-algebraic sets of complexity $O(1)$; the lines in $\Sigma_{1}^{\prime \prime}$ will be added to $\Sigma^{\prime}$; by Proposition 6.1, these lines can be covered by $O\left(s^{-2} h^{-O(1)}\right)=O\left(s^{-2} c^{-O(1)}\right)$ rectangular prisms of dimensions $2 \times s \times s \times s$.

Define $\Sigma^{\prime \prime}=\Sigma_{1}^{\prime}$ (recall that $\Sigma^{\prime}$ and $\Sigma^{\prime \prime}$ are the output of the lemma). We will show that these lines can be covered by $O\left(u^{-1}(s c)^{-O(1)}\right)$ rectangular prisms of dimensions $2 \times 2 \times u \times u$.

Define $Z_{2}=\left\{z \in Z_{1}: \Phi_{2}(z) \neq \emptyset\right\}$. Define

$$
A_{2}=\left\{(z, S) \in A_{1}: z \in Z_{2}\right\} .
$$


This is the set of pairs $(z, S)$ so that $z \in Z_{2}$ and all of the vectors from $\pi_{S}(\Phi(z))$ (and thus all of the vectors from $\pi_{S}\left(\Phi_{2}(z)\right)$ ) are contained in the $2 u$-neighborhood of $S$. In practice, it will be more convenient to work with the set

$$
\tilde{A}_{2}=\left\{\left(z, z+\operatorname{span}(S):(z, S) \in A_{2}\right\} ;\right.
$$

this is the set of pairs $(z, \Pi)$, where $\Pi$ is an affine 2-plane containing $z$ that is spanned by the vectors in $S(z)$.

Let $\mathcal{R}_{\text {max }}$ be a maximal set of essentially disjoint rectangular prisms of dimensions $2 \times 2 \times u \times u$ that intersect $B(0,1)$. For each $R \in \mathcal{R}_{\max }$, define $\Pi(R)$ to be the affine plane concentric with the long axes of $R$, and define

$$
R^{*}=\{(x, \Pi) \in R \times \operatorname{Grass}(4 ; 2): \operatorname{dist}(\Pi, \Pi(R)) \leq u\} .
$$

The expression $\operatorname{dist}(\Pi, \Pi(R))$ should be interpreted as follows: Select a semi-algebraic embedding of the affine Grassmannian Grass $(4 ; 2)$ into $\mathbb{R}^{N}$; then $\operatorname{dist}(\Pi, \Pi(R))$ is the Euclidean distance between the points in $\mathbb{R}^{N}$ corresponding to the images of $\Pi$ and $\Pi(R)$.

Observe that for each $C$, the $C$-fold dilates $\left\{C R^{*}\right\}_{R \in \mathcal{R}_{\max }}$ are $O_{C}(1)$-fold overlapping. For each $z \in Z_{2}$, there is a prism $R \in \mathcal{R}_{\max }$ so that $(z, \Pi(z)) \in R^{*}$. This value of $R$ satisfies

$$
R^{*} \cap \tilde{A}_{2} \neq \emptyset \text {. }
$$

For this $R$, we also have

$$
\Pi(z) \cap B(0,1) \subset 4 R
$$

where $4 R$ is the four-fold dilate or $R$. Note as well that if $(x, \ell) \in \Gamma_{1}$ with $x \in f_{Z}^{-1}(z)$, then $\ell$ is covered by $4 R$. This is because $\ell \cap 2 R \neq \emptyset$ and

$$
\begin{aligned}
\angle(\ell, \Pi(R)) & \leq \angle(\Pi(\ell), \Pi(z))+\angle(\Pi(z), \Pi(R)) \\
& \leq 2 u .
\end{aligned}
$$

Thus we must establish the bound

$$
\left|\left\{R \in \mathcal{R}_{\max }: R^{*} \cap \tilde{A}_{2} \neq \emptyset\right\}\right| \lesssim(h s)^{-O(1)} u^{-1} .
$$

By Lemma 5.1 .

$$
\mathcal{E}_{u}\left(\tilde{A}_{2}\right)=\mathcal{E}_{u}\left(A_{2}\right) \lesssim s^{-O(1)} u^{-3}
$$

Since the sets $\left\{C R^{*}: R \in \mathcal{R}_{\max }\right\}$ are $O_{C}(1)$ overlapping, in order to prove (36), it suffices to prove that if $R^{*} \cap \tilde{A}_{2} \neq \emptyset$, then

$$
\mathcal{E}_{u}\left(8 R^{*} \cap \tilde{A}_{2}\right) \gtrsim(h s)^{O(1)} u^{-2} .
$$


Suppose that $R^{*} \cap \tilde{A}_{2} \neq \emptyset$ and let $(z, \Pi) \in R^{*} \cap \tilde{A}_{2}$. Since $z \in 1-\operatorname{SBroad}_{s}\left(\Phi_{1}\right)$, there are $\geq s / u$ lines $\ell$ that point in pairwise $\geq u$ separated directions with $(x, \ell) \in \Gamma_{1}$ for some $x \in f_{Z}^{-1}(z)$. For each of these lines $\ell$, we have

$$
\begin{aligned}
\angle(\Pi(\ell), \Pi(R)) & \leq \angle(\Pi(\ell), \Pi(z))+\angle(\Pi(z), \Pi(R)) \\
& <4 u .
\end{aligned}
$$

On each of these lines, we have $\left|\Gamma_{1}(\ell)\right| \geq h$, so we can select $\geq h /(2 u)$ points that are all pairwise $u$ separated and have distance $\geq h / 2$ from $z$. For each such point $x^{\prime}$, we have

$$
\begin{aligned}
\angle\left(\Pi \circ f_{Z}\left(x^{\prime}\right), \Pi(R)\right) & \leq \angle\left(\Pi \circ f_{Z}\left(x^{\prime}\right), \Pi(\ell)\right)+\angle(\Pi(\ell), \Pi(R)) \\
& \leq 8 u .
\end{aligned}
$$

This gives us a set of $\geq h s /\left(2 u^{2}\right) \gtrsim(s c)^{O(1)} u^{-2}$ points $f_{Z}\left(x^{\prime}\right) \in Z_{1}$ that are pairwise $\gtrsim u$ separated, are contained in $Z_{1} \cap N_{8 u}(\Pi)$, and satisfy $\angle(\Pi(u), \Pi(R)) \leq 8 u$. This establishes (38).

\section{Understanding lines in $\Sigma_{2}$}

We will prove that if $w \geq(\text { cush } \kappa)^{O(1)}$ is sufficiently small, then the lines in $\Sigma_{2}$ can be covered by $O\left(s^{-2}\right)$ rectangular prisms of dimensions $2 \times s \times s \times s$.

Observe that for each $\ell \in \Sigma_{2}, \Gamma_{0}(\ell)$ has complexity $O(1)$. Thus if we select $h \gtrsim c$ sufficiently small, then there is an interval $I \subset \Gamma_{0}(\ell)$ of length $\geq 2 h$ so that $\Pi(I)$ is connected. Since $\ell \in \Sigma_{2}, \Pi(I)$ must also have diameter $\geq u$. We will now fix a value of $h$ so that this holds. Define

$$
\Gamma_{3}=\left\{(x, \ell) \in \Gamma_{0}: \ell \in \Sigma_{2}, \exists \text { an interval } x \in I \subset \Gamma_{0}(\ell) \text { of length } 2 h\right\} .
$$

Observe that $\left|\Gamma_{3}(\ell)\right| \geq 2 h \gtrsim c$ for all $\ell \in \Sigma_{2}$. For each $\ell \in \Sigma_{2}$, let $\gamma_{\ell} \subset \operatorname{Grass}(4 ; 2)$ be a connected set of diameter $\geq u$ that is contained in $\Pi \circ f_{Z}(\Gamma(\ell))$. Let $\Phi_{3}$ be the set associated to $\Gamma_{3}$, in the sense of Definition 5.4. Define

$$
\Phi_{3}^{\prime}=\left\{(z, v) \in \Phi_{3}: z \in 1-\operatorname{SBroad}_{s}\left(\Phi_{3}\right)\right\},
$$

and let $\Phi_{3}^{\prime \prime}=\Phi_{3} \backslash \Phi_{3}^{\prime}$. Let $\Gamma_{3}^{\prime}$ be the pre-image of $\Phi_{3}^{\prime}$ under the map $\Gamma_{3} \rightarrow \Phi_{3}$, and define $\Gamma_{3}^{\prime \prime}=\Gamma_{3} \backslash \Gamma_{3}^{\prime}$. Define

$$
\begin{aligned}
& \Sigma_{3}=\left\{\ell \in \Sigma_{2}:\left|\Gamma_{3}^{\prime}(\ell)\right| \geq h\right\}, \\
& \Sigma_{3}^{\prime}=\left\{\ell \in \Sigma_{2}:\left|\Gamma_{3}^{\prime \prime}(\ell)\right| \geq h\right\} .
\end{aligned}
$$


Arguing as above, the lines in $\Sigma_{3}^{\prime}$ can be covered by $O\left(c^{-O(1)} s^{-2}\right)$ rectangular prisms of dimensions $2 \times s \times s \times s$. We must now repeat the above process one more time. Define

$$
\Gamma_{4}=\left\{(x, \ell) \in \Gamma_{0}: \ell \in \Sigma_{3}\right\} .
$$

Let $\Phi_{4}$ be the set associated to $\Gamma_{4}$, in the sense of Definition 5.4. Define

$$
\Phi_{4}^{\prime}=\left\{(z, v) \in \Phi_{4}: z \in 1-\operatorname{SBroad}_{s}\left(\Phi_{4}\right)\right\},
$$

and let $\Phi_{4}^{\prime \prime}=\Phi_{4} \backslash \Phi_{4}^{\prime}$. Let $\Gamma_{4}^{\prime}$ be the pre-image of $\Phi_{4}^{\prime}$ under the map $\Gamma_{4} \rightarrow \Phi_{4}$, and define $\Gamma_{4}^{\prime \prime}=\Gamma_{4} \backslash \Gamma_{4}^{\prime}$. Define

$$
\begin{aligned}
& \Sigma_{4}=\left\{\ell \in \Sigma_{3}:\left|\Gamma_{4}^{\prime}(\ell)\right| \geq h / 2\right\}, \\
& \Sigma_{4}^{\prime}=\left\{\ell \in \Sigma_{3}:\left|\Gamma_{4}^{\prime \prime}(\ell)\right| \geq h / 2\right\} .
\end{aligned}
$$

Again, the lines in $\Sigma_{4}^{\prime}$ can be covered by $O\left(c^{-O(1)} s^{-2}\right)$ rectangular prisms of dimensions $2 \times s \times s \times s$. We will show that if $w \geq(\operatorname{csu\kappa })^{O(1)}$ is sufficiently small, then $\Sigma_{4}$ is empty. The basic idea is as follows: If $\Sigma_{4}$ is not empty, then we will find lines $\ell_{0}$ and $\ell^{\prime}$ that are (quantitatively) skew so that there are many (i.e. about $w^{-1}$ ) points $x \in \ell_{0}$ where the plane $\Pi \circ f_{Z}(x)$ intersects $\ell^{\prime}$. This implies that the plane $\Pi \circ f_{Z}(x)$ is contained in the hyperplane $H$ spanned by $\ell$ and $\ell^{\prime}$. Each of these planes $\Pi \circ f_{Z}(x)$ contains many lines (almost) contained in $\Sigma_{2}$, which will imply that the $w$-neighborhood of these planes each intersect $N_{w}(Z)$ in a set of measure roughly $w^{2}$. Since there are roughly $w^{-1}$ such planes, this implies that $\left|N_{w}(H) \cap N_{w}(Z)\right|$ has size roughly $w$. This contradicts the fact that $\|I I(x)\|_{\infty} \geq \kappa$ on $Z$, which implies that $\left|N_{w}(H) \cap N_{w}(Z)\right|$ has size at most $\kappa^{-1 / 2} w^{3 / 2}$.

We will now make this argument precise. For each $\ell \in \Sigma_{2}$, let $T_{\ell}$ be the $w$ neighborhood of $\ell \cap B(0,1)$, and let $Y_{2}\left(T_{\ell}\right) \subset T$ be the $w$-neighborhood of $\Gamma_{0}(\ell)$. Observe that $\left|Y_{2}\left(T_{\ell}\right)\right| \gtrsim c\left|T_{\ell}\right|$ for each such $\ell \in \Sigma_{2}$. Let $\mathbb{T}_{2}$ be a maximal set of essentially distinct tubes from $\left\{T_{\ell}: \ell \in \Sigma_{2}\right\}$.

Similarly, for each $\ell \in \Sigma_{3}$, let $T_{\ell}$ be the $w$-neighborhood of $\ell \cap B(0,1)$, and let $Y_{3}\left(T_{\ell}\right) \subset T$ be the $w$-neighborhood of $\Gamma_{3}(\ell)$. Again, we have $\left|Y_{3}\left(T_{\ell}\right)\right| \gtrsim c\left|T_{\ell}\right|$ for each such $\ell \in \Sigma_{3}$. Let $\mathbb{T}_{3}$ be a maximal set of essentially distinct tubes from $\left\{T_{\ell}: \ell \in \Sigma_{3}\right\}$.

Remark 6.3. Observe that for every $T \in \mathbb{T}_{3}$ and every plane $\Pi$ containing the line coaxial with $T$, there are $\gtrsim(s c)^{O(1)} w^{-2}$ tubes $T^{\prime} \in \mathbb{T}_{2}$ with $T \cap T^{\prime} \neq \emptyset$, $\angle\left(v(T), v\left(T^{\prime}\right)\right) \gtrsim(s c h)^{O(1)}$, and $\angle\left(v\left(T^{\prime}\right), \Pi\right) \gtrsim(u c h)^{O(1)}$.

Our next task is to establish the bound

$$
\left|\mathbb{T}_{2}\right| \lesssim c^{-O(1)} w^{-3} .
$$


For each $x \in Z$, define

$$
\mathbb{T}_{2}(x)=\left\{T \in \mathbb{T}_{2}: x \in Y_{2}(T)\right\} .
$$

For each $T \in \mathbb{T}_{2}$, define $v(T)=v(\ell)$, where $\ell$ is the line coaxial with $T$, and define

$$
T^{*}=\left\{(x, v) \in \mathbb{R}^{4} \times S^{3}: x \in T, \angle(v, v(T)) \leq w\right\} .
$$

Then the sets $\left\{T^{*}\right\}_{T \in \mathbb{T}_{2}}$ are $O(1)$ overlapping.

Recall the set $A_{1}$ from (31). By Lemma 5.1, $\mathcal{E}_{w}\left(A_{1}\right) \lesssim s^{-O(1)} w^{-3}$. Define

$$
G=\left\{(z, v) \in Z_{1} \times S^{3}: v \in S(z)\right\} .
$$

By Lemma 2.3, we have

$$
\mathcal{E}_{w}(G) \lesssim s^{-O(1)} w^{-4} .
$$

On the other hand, if $T_{\ell} \in \mathbb{T}_{2}$, then there are $\gtrsim \frac{c}{w} w$-separated points $z \in T_{\ell} \cap Z_{1}$ with $\angle(v(\ell), \Pi(z)) \leq w$ and thus $(z, v(T)) \in G$. Thus if $T \in \mathbb{T}_{2}$, we have

$$
N_{w}\left(T^{*} \cap G\right) \gtrsim c w^{-1} .
$$

Combining (40) and (41), we obtain (39).

We will now show that $\Sigma_{4}$ is empty. Suppose not. Let $\ell_{0} \in \Sigma_{4}$. Let $T_{0}$ be the $w$-neighborhood of $\ell \cap B(0,1)$, and let $Y_{4}(T)$ be the $w$-neighborhood of $\Gamma_{4}^{\prime}\left(\ell_{4}\right)$. Let $z_{1}, \ldots, z_{p}, p \gtrsim(c s u)^{O(1)} w^{-1}$ be points in $f_{Z}\left(\Gamma_{4}^{\prime}\left(\ell_{0}\right)\right) \subset Y_{4}\left(T_{0}\right)$ so that the planes $\Pi\left(z_{i}\right)$ point in $w$-separated directions. (Recall that each of these planes makes an angle $\leq w$ with $\left.v\left(\ell_{0}\right)=v\left(T_{0}\right)\right)$

For each $i=1, \ldots, p$, since $z_{i} \in Y_{4}\left(T_{0}\right)$, we can select a set $\mathbb{T}^{(i)}$ of $\gtrsim(c s)^{O(1)} w^{-1}$ tubes from $\mathbb{T}_{3}$ passing through $z_{i}$, so that each of these tubes makes an angle $\gtrsim$ $(c s)^{O(1)}$ with $v\left(T_{0}\right)$. Since the set $\mathbb{T}^{(i)}$ is contained in the $s^{-O(1)} w$-neighborhood of the plane $\Pi\left(z_{i}\right)$, and these planes point in $w$-separated directions, we can refine the set of indices $1, \ldots, p$ to a new indexing set $1, \ldots, p^{\prime}$ with $p^{\prime} \gtrsim(c s u)^{O(1)} w^{-1}$ so that the corresponding sets of tubes $\left\{\mathbb{T}^{(i)}\right\}_{i=1}^{p^{\prime}}$ are disjoint. As discussed in Remark 6.3 . for each index $i$ and each $T \in \mathbb{T}^{(i)}$, there exist $\gtrsim(\operatorname{csu})^{O(1)} w^{-2}$ tubes from $\mathbb{T}$ that intersect $T$, make an angle $\gtrsim(c s u)^{O(1)}$ with $v(T)$, and that make an angle $\gtrsim(c s u)^{O(1)}$ with the plane spanned by $v\left(T_{0}\right)$ and $v(T)$ (and thus make an angle $\gtrsim(c s u)^{O(1)}$ with the plane $\left.\Pi\left(z_{i}\right)\right)$. We can also require that each of these tubes intersect $T$ in a point that has distance $\gtrsim(c s u)^{O(1)}$ from $T_{0}$, i.e. each of these tubes is $\gtrsim(c s u)^{O(1)}$ skew to $T_{0}$. 
Thus if $C=O(1)$ is chosen sufficiently large, there are $\gtrsim(c s u)^{O(1)} w^{-4}$ pairs

$$
\begin{aligned}
\left\{\left(T, T^{\prime}\right) \in \mathbb{T}_{3} \times \mathbb{T}_{2}:\right. & T_{0} \cap T \neq \emptyset, \angle\left(v\left(T_{0}\right), v(T)\right) \gtrsim(c s u)^{C}, \\
& T \cap T^{\prime} \neq \emptyset, \angle\left(v(T), v\left(T^{\prime}\right)\right) \gtrsim(c s u)^{C}, \\
& \left.T \text { and } T^{\prime} \text { are } \gtrsim(c s u)^{C} \text { skew }\right\} .
\end{aligned}
$$

Since $\left|\mathbb{T}_{2}\right| \lesssim c^{-O(1)} w^{-3}$, we can select a tube $T^{\prime} \in \mathbb{T}_{2}$ that is $\gtrsim(c s u)^{C}$ skew to $T_{0}$, so that there are $\gtrsim(c s u)^{O(1)} w^{-1}$ tubes $T \in \mathbb{T}_{3}$ with $\left(T, T^{\prime}\right) \in(42)$. Note that at most $\lesssim(c s u)^{-1}$ of these tubes $T \in \mathbb{T}_{3}$ can lie in the $w$-neighborhood of a common plane containing $v\left(T_{0}\right)$, since $T^{\prime}$ is $\gtrsim(c s u)^{C}$ skew to $T_{0}$. Thus we can select $\gtrsim(c s u)^{O(1)} w^{-1}$ tubes $T$ with $\left(T, T^{\prime}\right) \in(42)$ so that the planes $\left\{\operatorname{span}\left(v\left(T_{0}\right), v(T)\right\}\right.$ point in $w$-separated directions.

Let $H$ be the hyperplane containing the lines coaxial with $T_{0}$ and $T^{\prime}$. Observe that if $\left(T, T^{\prime}\right) \in(42)$, and if $x \in T_{0} \cap T$, then $\angle(\Pi(x), H) \lesssim(c s u)^{-O(1)} w$. Re-indexing the sets of tubes $\left\{\mathbb{T}^{(1)}, \ldots, \mathbb{T}^{\left(p^{\prime}\right)}\right\}$ again, we can select sets $\mathbb{T}^{(1)}, \ldots, \mathbb{T}^{\left(p^{\prime \prime}\right)}, p^{\prime \prime} \gtrsim$ $(c s u)^{O(1)} w^{-1}$ so that every set of tubes $\mathbb{T}^{(i)}$ contains a tube $T$ with $\left(T, T^{\prime}\right) \in(42)$. This means that for each index $i$, the tubes in $\mathbb{T}^{(i)}$ are contained in the $\lesssim(c s u)^{-O(1)} w-$ neighborhood of the hyperplane $H$ (see Figure 2). We have

$$
\left|\bigcup_{i=1}^{p^{\prime \prime}} \bigcup_{T \in \mathbb{T}^{(i)}} Y_{2}(T)\right| \gtrsim(c s u)^{O(1)} w .
$$

Since $Y_{2}(T) \subset N_{w}(Z)$ for each tube $T$ in the above union, we have

$$
\left|N_{w}(Z) \cap N_{\lesssim(c s u)^{-O(1)} w}(H)\right| \gtrsim(c s u)^{O(1)} w .
$$

But by Lemma 4.3 , we have

$$
\left|N_{w}(Z) \cap N_{\lesssim(c s u)^{-O(1)} w}(H)\right| \lesssim(c s u)^{-O(1)} \kappa^{-1 / 2} w^{3 / 2}
$$

and thus

$$
(c s u)^{O(1)} w \lesssim(c s u)^{-O(1)} \kappa^{-1 / 2} w^{3 / 2} .
$$

If $w \gtrsim(c \kappa u s)^{O(1)}$ is chosen sufficiently small, then 43 is impossible, which contradicts the assumption that $\Sigma_{4} \neq \emptyset$. We conclude that $\Sigma_{4}=\emptyset$, which completes the proof of Lemma 6.1.

Lemma 6.1 can be used to understand hypersurfaces that are doubly-ruled by planes. 


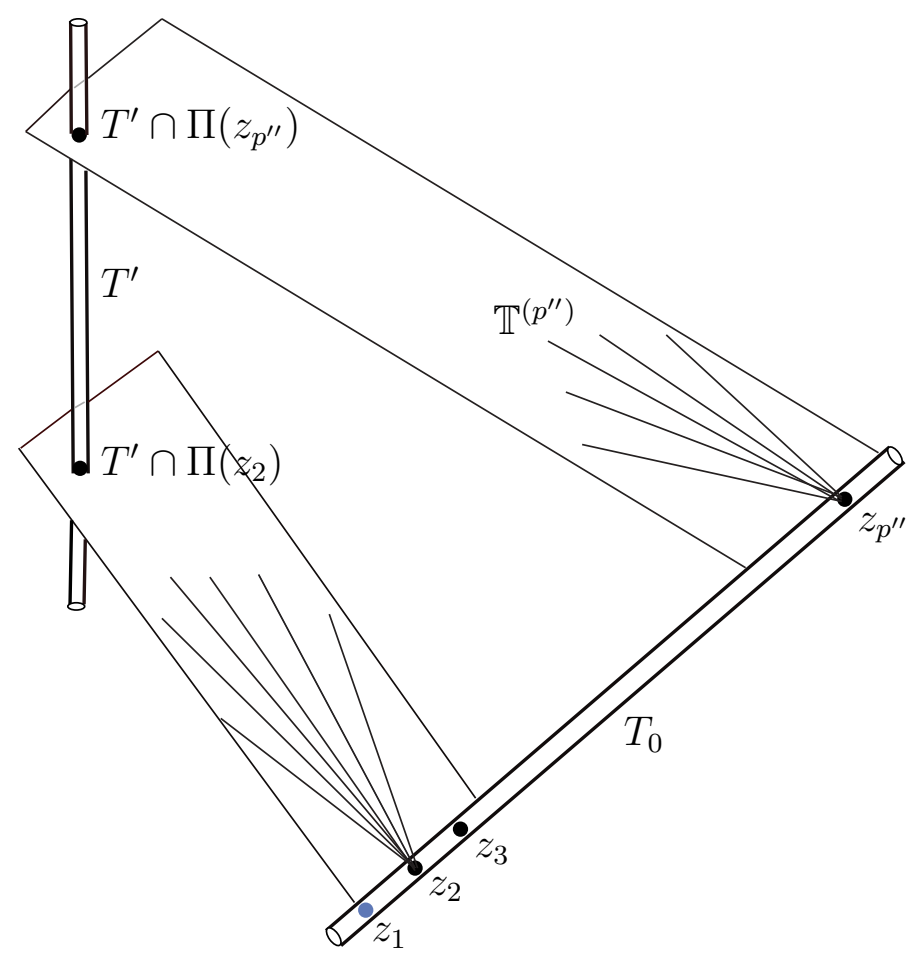

Figure 2: The tubes $T_{0}$ and $T^{\prime}$; the points $z_{1}, \ldots, z_{p^{\prime \prime}} \in T_{0}$, the planes $\Pi\left(z_{1}\right), \ldots, \Pi\left(z_{p^{\prime \prime}}\right)$, and the sets of tubes $\mathbb{T}^{(1)}, \ldots, \mathbb{T}^{\left(p^{\prime \prime}\right)}$. This entire figure is ostensibly contained in $\mathbb{R}^{4}$, but in fact it is contained in the $\lesssim(c s u)^{-O(1)} w$-neighborhood of the hyperplane spanned by the lines coaxial with $T_{0}$ and $T^{\prime}$.

Proposition 6.2. Let $\delta, u, s, \kappa, c, D, E$ be parameters with $0<\delta<u<s<c$ and $\delta<\kappa$. Then there exists a number $w \gtrsim_{D, E}(u s \kappa c)^{O(1)}$ so that the following holds.

Let $P \in \mathbb{R}\left[x_{1}, \ldots, x_{4}\right]$ be a polynomial of degree at most $D$ and let

$$
Z \subset\left\{z \in Z(P) \cap B(0,1): 1 \leq|\nabla P(z)| \leq 2,\|I I(z)\|_{\infty} \geq \kappa\right\} .
$$

Let $\Sigma \subset \Sigma_{\delta, c}(Z)$, and let $\Gamma \subset \Gamma\left(N_{\delta}(Z), \Sigma\right)$. Suppose that $Z, \Sigma$, and $\Gamma$ are semialgebraic sets of complexity at most $E$. Let $\Phi$ be associated to $\Gamma$, in the sense of Definition 5.4. Suppose that

$$
\begin{aligned}
& Z \subset(2,2)-\operatorname{Narrow}_{w}(\Phi), \\
& |\Gamma(\ell)| \geq c \quad \text { for all } \ell \in \Sigma .
\end{aligned}
$$

Then we can write $\Sigma=\Sigma^{\prime} \cup \Sigma^{\prime \prime}$, where the lines in $\Sigma^{\prime}$ can be covered by $O_{D, E}\left(c^{-O(1)} s^{-2}\right)$ rectangular prisms of dimensions $2 \times s \times s \times s$, and the lines in $\Sigma^{\prime \prime}$ can be covered by $O_{D, E}\left((c s)^{-O(1)} u^{-1}\right)$ rectangular prisms of dimensions $2 \times 2 \times u \times u$. 
Proof. Let $w \gtrsim_{D, E}(u s \kappa c)^{O(1)}$ be the constant from Lemma 6.1 associated to the values $\delta^{\prime}=\delta, u^{\prime}=u, s^{\prime}=s, \kappa^{\prime}=\kappa, c^{\prime}=c / 2, D^{\prime}=D, E^{\prime}=O_{D, E}(1)$. Define

$$
A=\left\{\left(z, S_{1}, S_{2}\right) \in Z \times\left(\mathcal{S}_{1}\right)^{2}: \min \left(\angle\left(v, S_{1}\right), \angle\left(v, S_{2}\right)\right) \leq w \forall v \in \pi_{S}(\Phi(z))\right\} .
$$

Since $Z \subset(2,2)-\operatorname{Narrow}_{w}(\Phi)$, the projection $A \mapsto Z$ is onto. Use Lemma 2.6 to select a set $A^{\prime} \subset A$ so that the map $\pi: A^{\prime} \rightarrow Z$ is a bijection. Define the semi-algebraic functions $S_{1}(z)$ and $S_{2}(z): Z \rightarrow \mathcal{S}_{1}$ so that for each $z \in Z,\left(z, S_{1}(z), S_{2}(z)\right) \in A^{\prime}$. For $i=1,2$, define

$$
\Gamma_{i}=\left\{(x, \ell) \in \Gamma: \angle\left(v(\ell), S_{i} \circ f_{Z}(x) \leq w\right\} .\right.
$$

By (45), for every $(x, \ell) \in \Gamma$ we have that

$$
\angle\left(v(\ell), S_{1} \circ f_{Z}(x)\right) \leq w \quad \text { and } / \text { or } \quad \angle\left(v(\ell), S_{2} \circ f_{Z}(x)\right) \leq w .
$$

Thus $\Gamma=\Gamma_{1} \cup \Gamma_{2}$. For $i=1,2$, define

$$
\Sigma_{i}=\left\{\ell \in \Sigma:\left|\Gamma_{i}(\ell)\right| \geq c / 2\right\} .
$$

Then $\Sigma=\Sigma_{1} \cup \Sigma_{2}$. We have that $Z, \Gamma_{i}$, and $\Sigma_{i}, i=1,2$, are semi-algebraic of complexity $E^{\prime}=O_{D, E}(1)$. For $i=1,2$, apply Lemma 6.1 to the data $P, Z, \Sigma_{i}, \Gamma_{i}$, with the parameters $\delta^{\prime}, u^{\prime}, s^{\prime}, \kappa^{\prime}, c^{\prime}, D^{\prime}$, and $E^{\prime}$ described above, and let $\Sigma_{i}^{\prime}$ and $\Sigma_{i}^{\prime \prime}$ be the output from the lemma. Define $\Sigma^{\prime}=\Sigma_{1}^{\prime} \cup \Sigma_{2}^{\prime}$ and define $\Sigma^{\prime \prime}=\Sigma_{1}^{\prime \prime} \cup \Sigma_{2}^{\prime \prime}$.

\section{Broad varieties}

In this section, we will consider the region where $Z$ is robustly 1-broad, (2, 2)-broad, and has large second fundamental form. We will show that many of the lines that have large intersection with this region must lie near a quadratic hypersurface; this will be the set of lines $\Sigma_{4}^{\prime}$ from the statement of Theorem 1.2 . This result will be proved in Proposition 7.1, which is the main result of this section.

\subsection{Neighborhoods of quadratic curves}

Lemma 7.1. Let $Q\left(x_{1}, x_{2}\right)=a_{11} x_{1}^{2}+a_{22} x_{2}^{2}+a_{12} x_{1} x_{2}$ be a monic quadratic polynomial. Let

$$
\begin{aligned}
& S_{1}=S^{2} \cap Z\left(\left(a_{12}+\mathcal{R}\left[\left(a_{12}^{2}-4 a_{11} a_{22}\right)^{1 / 2}\right]\right) x_{1}+2 a_{22} x_{2}\right), \\
& S_{2}=S^{2} \cap Z\left(\left(a_{12}-\mathcal{R}\left[\left(a_{12}^{2}-4 a_{11} a_{22}\right)^{1 / 2}\right]\right) x_{1}+2 a_{22} x_{2}\right),
\end{aligned}
$$


where $\mathcal{R}[z]$ is the real part of $z$. Then there is an absolute constant $C$ so that for each $t>0$,

$$
\left\{x \in S^{2}:|Q(x)| \leq t\right\} \subset N_{C t^{1 / 2}}\left(S_{1} \cup S_{2}\right) .
$$

Remark 7.1. The requirement that $Q$ be monic can be replaced by the condition that the largest coefficient of $Q$ has magnitude $A>0$. Then the constant $C$ in (47) depends on $A$.

Definition 7.1. Let $Q \in \mathbb{R}\left[x_{1}, x_{2}, x_{3}\right]$ be a homogeneous polynomial of degree 2 . We say that $Q$ is $w$-degenerate if there exist great circles $S_{1}, S_{2} \subset S^{2}$ so that $Z(Q) \cap S^{2} \subset$ $N_{w}\left(S_{1} \cup S_{2}\right)$. If $Q$ is not $w$-degenerate, then we call it $w$-non-degenerate.

Lemma 7.2. Let $Q \in \mathbb{R}\left[x_{1}, x_{2}, x_{3}\right]$ be a homogeneous quadratic polynomial. Suppose that $Q$ is $w$-non-degenerate. Then for every $t<w$ and for every great circle $S \subset S^{2}$, we have

$$
\left|S_{2} \cap Z(Q) \cap N_{t}(S)\right| \lesssim(t / w)^{1 / 2},
$$

where $|\cdot|$ denotes one-dimensional Lebesgue measure.

Lemma 7.3. Let $Q\left(x_{1}, x_{2}, x_{3}\right)$ be a monic homogeneous polynomial of degree 2 and let $w>0$. Then there is an absolute constant $c>0$ so that at least one of the following two things must hold

1. There exist two great circles $S_{1}, S_{2} \subset S^{2}$ so that

$$
\left\{x \in S^{2}:|Q(x)| \leq c w^{2}\right\} \subset N_{w}\left(S_{1} \cup S_{2}\right) .
$$

2. For each $t>0$, we have

$$
\left\{x \in S^{2}:|Q(x)| \leq c t\right\} \subset N_{t / w^{2}}(Z(Q)) .
$$

Proof. Let $c_{1}>0$ be a constant to be determined later. We will consider two cases. Case (A): there exists a point $p \in Z(Q) \cap S^{2} \subset \mathbb{R}^{3}$ where the map $x \mapsto Q(x)$ has small derivative, i.e. $|D Q(p)| \leq c_{1} w^{2}$. We will show that Item 1 must hold. After a rotation, we can assume that $p=(1,0,0)$. After applying this rotation, we have

$$
Q\left(x_{1}, x_{2}, x_{3}\right)=a_{22} x_{2}^{2}+a_{33} x_{3}^{2}+a_{12} x_{1} x_{2}+a_{13} x_{1} x_{3}+a_{23} x_{2} x_{3},
$$

and

$$
D Q(p)=\left(\partial_{x_{2}} Q(1,0,0), \partial_{x_{3}} Q(1,0,0)\right)=\left(a_{12}, a_{13}\right)
$$

Thus

$$
Q\left(x_{1}, x_{2}, x_{3}\right)=a_{22} x_{2}^{2}+a_{33} x_{3}^{2}+a_{23} x_{2} x_{3}+O\left(c_{1} w^{2}\right)\left(x_{1} x_{2}+x_{1} x_{3}\right),
$$


where at least one of $a_{22}, a_{33}, a_{23}$ has magnitude $\sim 1$.

By Lemma 7.1, we have that if $c_{2}>0$ is chosen sufficiently small (independent of $\left.c_{1}\right)$, then

$$
\left\{x \in S^{2}:\left|a_{22} x_{2}^{2}+a_{33} x_{3}^{2}+a_{23} x_{2} x_{3}\right| \leq 10 c_{2} w^{2}\right\} \subset N_{w}\left(S_{1} \cup S_{2}\right),
$$

where

$$
\begin{aligned}
& S_{1}=S^{2} \cap Z\left(\left(a_{23}+\mathcal{R}\left[\left(a_{23}^{2}-4 a_{22} a_{33}\right)^{1 / 2}\right]\right) x_{2}+2 a_{33} x_{3}\right), \\
& S_{2}=S^{2} \cap Z\left(\left(a_{23}-\mathcal{R}\left[\left(a_{23}^{2}-4 a_{22} a_{33}\right)^{1 / 2}\right]\right) x_{2}+2 a_{33} x_{3}\right) .
\end{aligned}
$$

Next, if $c_{1}>0$ is chosen sufficiently small (depending on $c_{2}$ ), then

$$
\left\{x \in S^{2}:|Q(x)| \leq c_{2} w^{2}\right\} \subset\left\{x \in S^{2}:\left|a_{22} x_{2}^{2}+a_{33} x_{3}^{2}+a_{23} x_{2} x_{3}\right| \leq 10 c_{2} w^{2}\right\},
$$

which completes the analysis of Case (A).

Now suppose we are in Case (B): $|D Q(p)| \geq c_{1} w^{2}$ for all $p \in Z(Q) \cap S^{2}$. Since $Q$ is quadratic, $D Q: \mathbb{R}^{3} \rightarrow \mathbb{R}^{3}$ is a linear map. If $c_{3}>0$ is chosen sufficiently small (depending on $c_{1}$ ), then $|Q(x)| \leq c_{3} t$ implies $\operatorname{dist}(t, Z(Q)) \leq t / w^{2}$. Thus Item 2 must hold.

To complete the proof, choose $c=\min \left(c_{2}, c_{3}\right)$.

Lemma 7.4. Let $P \in \mathbb{R}\left[x_{1}, x_{2}, x_{3}, x_{4}\right]$ be a polynomial of degree at most $D$. Let

$$
Z \subset\left\{x \in Z(P) \cap B(0,1), 1 \leq|\nabla P(x)| \leq 2,\|I I(x)\|_{\infty} \geq \kappa\right\} .
$$

Let $\Gamma \subset \Gamma\left(N_{\delta}(Z), \mathcal{L}\right)$ and let $\Phi \subset Z \times S^{3}$ be associated to $\Gamma$, in the sense of Definition 5.4. Suppose that

- $Z \subset(2,2)-\operatorname{Broad}_{w}(\Phi)$.

- For each $z \in Z$ and each $v \in \pi_{S}(\Phi(z))$, we have

$$
\left|(v \cdot \nabla)^{j} P(z)\right| \leq K \delta, \quad j=1,2 .
$$

Then for each $z \in Z$, the vectors in $\pi_{S}(\Phi(z))$ are contained in the $\delta(K /(w \kappa))^{O(1)}$ neighborhood of the set

$$
\mathcal{C}_{z}=\left\{v \in S^{3}:(v \cdot \nabla) P(z)=0,(v \cdot \nabla)^{2} P(z)=0\right\} .
$$


Proof. Let $x \in Z$. After a translation and rotation, we can assume that $x=0$ and $N(0)=(1,0,0,0)$. Then we can expand

$$
P\left(x_{1}, x_{2}, x_{3}, x_{4}\right)=x_{1}+\sum_{\substack{|I|=2 \\ \text { yes } x_{1}}} a_{I} x^{I}+\sum_{\substack{|I|=2 \\ \text { no } x_{1}}} a_{I} x^{I}+\sum_{|I|>2} a_{I} x^{I},
$$

where the first sum is taken over all multi-indices $I$ of length two that include at least one $x_{1}$ term, and the second sum includes all the other multi-indices of length two.

Let $v \in \pi_{S}(\Phi(x))$; we can write $v=\left(v_{1}, v_{2}, v_{3}, v_{4}\right)$. Since $v$ satisfies (48), we have $\left|v_{1}\right| \leq K \delta$. Define

$$
A=\|I I(0)\|_{\infty}^{-1}\left[\begin{array}{lll}
a_{22} & a_{23} & a_{24} \\
a_{23} & a_{33} & a_{34} \\
a_{24} & a_{34} & a_{44}
\end{array}\right] .
$$

Since $v$ satisfies (48) and $\|I I(0)\|_{\infty} \geq \kappa$, we have

$$
\left|\left[v_{2}, v_{3}, v_{4}\right]^{T} A\left[v_{2}, v_{3}, v_{4}\right]\right| \lesssim \kappa^{-1} K \delta
$$

Now consider the function

$$
Q\left(v_{1}, v_{2}, v_{3}\right)=\left[v_{2}, v_{3}, v_{4}\right]^{T} A\left[v_{2}, v_{3}, v_{4}\right] .
$$

Since $0 \in(2,2)-\operatorname{Broad}_{w}(\Phi)\left(\right.$ remember, originally we had $z \in(2,2)-\operatorname{Broad}_{w}(\Phi)$, but we applied a translation sending $z$ to 0$)$, the set of unit vectors $\left(v_{2}, v_{3}, v_{4}\right)$ satisfying (50) cannot be contained in the $w$-neighborhood of the union of two great circles in $S^{2}$. Thus by Lemma 7.3 , we have that

$$
\left(v_{2}, v_{3}, v_{4}\right) \in S^{2} \cap N_{c \kappa^{-1} K \delta / w^{2}}(Z(Q)),
$$

where $c>0$ is an absolute constant. Thus

$$
v \subset N_{t}\left(\mathcal{C}_{z}\right), \quad \text { where } t \lesssim \delta K /\left(\kappa w^{2}\right) .
$$

Definition 7.2. In (49) above, we defined the set

$$
\mathcal{C}_{z}=\left\{v \in S^{3}:(v \cdot \nabla) P(z)=0,(v \cdot \nabla)^{2} P(z)=0\right\} .
$$

We will call this the quadratic cone of $Z(P)$ with vertex $z$. More generally, any set of the form (51) will be called a quadratic cone. Following Definition 7.1, we say that the quadratic cone $\mathcal{C}_{z}$ is $w$-degenerate if there exist great circles

$$
S_{1}, S_{2} \subset\left\{v \in S^{3}:(v \cdot \nabla) P(z)=0\right\}
$$


so that $\mathcal{C}_{z} \subset N_{w}\left(S_{1} \cup S_{2}\right)$. Otherwise we say $\mathcal{C}_{z}$ is $w$-non-degenerate.

Define

$$
\tilde{\mathcal{C}}_{z}=z+\operatorname{span}\left(\mathcal{C}_{z}\right)
$$

this is a two-dimensional algebraic variety in $\mathbb{R}^{4}$; it is the union of all lines that intersect $z$ and also intersect the curve $z+\mathcal{C}_{z}$. We say that $\tilde{\mathcal{C}}_{z}$ is $w$-non-degenerate if $\mathcal{C}_{z}$ is $w$-non-degenerate. Observe that $\tilde{\mathcal{C}}_{z}$ is a degree-two algebraic surface; it can be defined as the common zero locus of a degree one and a degree two polynomial in $\mathbb{R}\left[x_{1}, x_{2}, x_{3}, x_{4}\right]$. If $\mathcal{C}_{z}$ is a quadratic cone, $\ell \in \mathcal{L}, z \in \ell$, and $\operatorname{dist}\left(v(\ell), \mathcal{C}_{z}\right)=t$, then $\ell \cap B(0,1) \subset N_{t}\left(\tilde{\mathcal{C}}_{z}\right)$.

\subsection{Unions of tubes}

For the next lemma, we will introduce some standard notation from the Kakeya problem. This notation will be used throughout the remainder of this section. Let $\mathbb{T}$ be a set of essentially distinct $\delta$-tubes, i.e. a set of $\delta$-neighborhoods of unit line segments so that no tube is contained in the two-fold dilate of any other. For each tube $T \in \mathbb{T}$, let $Y(T) \subset T$. For each $x \in \mathbb{R}^{4}$, define

$$
\mathbb{T}(x)=\{T \in \mathbb{T}: x \in Y(T)\}
$$

If the set $Y$ is ambiguous, we will sometimes use the notation $\mathbb{T}_{Y}(x)$ in place of $\mathbb{T}(x)$. For each $T \in \mathbb{T}$, define $v(T)$ to be the direction of the line coaxial with $T$. Thus for example

$$
v(\mathbb{T}(x))=\{v(T): T \in \mathbb{T}(x)\}
$$

For each $T_{0} \in \mathbb{T}$, define

$$
H\left(T_{0}\right)=\left\{T \in \mathbb{T}: Y\left(T_{0}\right) \cap Y(T) \neq \emptyset\right\}
$$

If the set $Y$ is ambiguous, we will sometimes use the notation $H_{Y}\left(T_{0}\right)$ in place of $H\left(T_{0}\right)$.

The next lemma says that if $\mathbb{T}$ is a set of tubes, and if the tubes passing through a typical point lie near a non-degenerate cone, then the tubes in a typical hairbrush are mostly disjoint and thus their union has large volume. This is a variant of Wolff's "hairbrush argument" from [22]. However, unlike in [22] we do not assume that the tubes point in different directions.

Lemma 7.5. Let $\delta, \lambda, t>0$. Let $\mathbb{T}$ be a set of essentially distinct $\delta$-tubes. For each $T \in \mathbb{T}$, let $Y(T) \subset T$ with $Y(T) \geq \lambda|T|$. Let $T_{0} \in \mathbb{T}$. Suppose that $\left|H\left(T_{0}\right)\right| \geq$ 
$t \delta^{-2}$ and that for every $x \in Y\left(T_{0}\right)$, the vectors $v(\mathbb{T}(x))$ are contained in the $K \delta$ neighborhood of a w-non-degenerate cone $\mathcal{C}_{x}$. Then

$$
\left|\bigcup_{T \in H\left(T_{0}\right)} Y(T)\right| \geq(w \lambda t / K)^{O(1)} \delta .
$$

Proof. By pigeonholing, we can select a set of $\geq t \lambda / 2 \delta$ points $x \in Y\left(T_{0}\right)$ that are $\delta$ separated and that satisfy $|\mathbb{T}(x)| \geq \frac{1}{2} t \lambda \delta^{-1}$. The line coaxial with $T_{0}$ passes through $x$ and makes an angle $\leq K \delta$ with a line $\tilde{\ell}$ in the cone $\tilde{\mathcal{C}}_{x}$ (see Figure 3). Let $\Pi_{x}$ be the plane that is tangent to $\tilde{\mathcal{C}}_{x}$ along $\tilde{\ell}$.

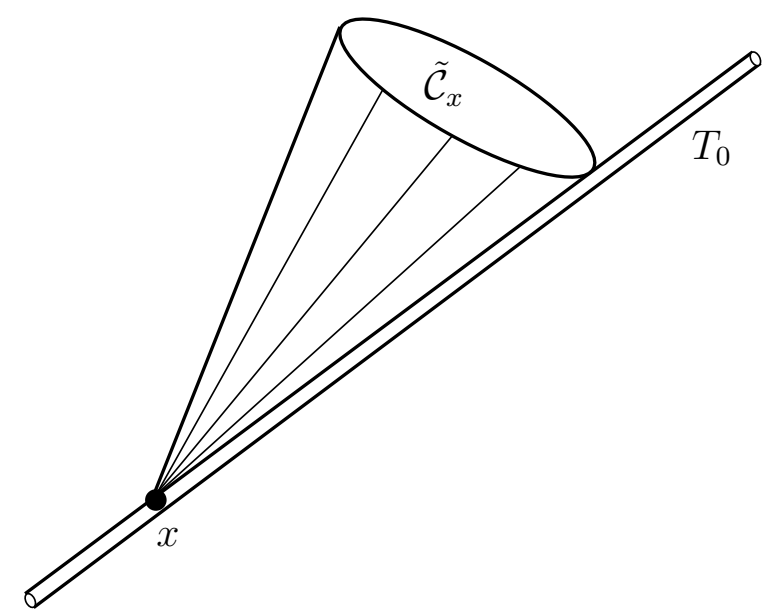

Figure 3: The tube $T_{0}$, the point $x$, and the cone $\tilde{\mathcal{C}}_{x}$. The plane $\Pi_{x}$ (not pictured) contains the line coaxial with $T_{0}$ and is tangent to $\tilde{\mathcal{C}}_{x}$.

Let $p=\frac{1}{16} w t^{2} \lambda^{2} K^{-2}$, and let

$$
\mathbb{T}(x)^{\prime}=\{T \in \mathbb{T}(x): \angle(v(T), \Pi(x)) \geq p\} .
$$

Since $\mathcal{C}_{x}$ is $w$-non-degenerate, by Lemma 7.2 , we have that

$$
\left|\left\{v \in S^{2}: \angle\left(v, \Pi_{x}\right) \leq p, v \in N_{K \delta}\left(\mathcal{C}_{x}\right)\right\}\right| \lesssim(K \delta)(p / w)^{1 / 2},
$$

where $|\cdot|$ denotes two-dimensional Haar measure on the sphere $S^{2}$; this set can contain at most $K(p / w)^{1 / 2} \delta^{-1} \delta$-separated points on $S^{2}$, which implies that

$$
\begin{aligned}
\left|\mathbb{T}(x) \backslash \mathbb{T}^{\prime}(x)\right| & \leq K(p / w)^{1 / 2} \delta^{-1} \\
& \leq|\mathbb{T}(x)| / 2,
\end{aligned}
$$


and thus $\left|\mathbb{T}^{\prime}(x)\right| \geq \frac{1}{4} t \lambda \delta^{-1}$ for each of the values of $x$ chosen above. Furthermore, for every plane $\Pi$ containing the line coaxial with $T$, we have that

$$
\left|\left\{T \in \mathbb{T}^{\prime}(x): T \subset N_{10 \delta}(\Pi)\right\}\right| \lesssim(K /(w \lambda t))^{O(1)} .
$$

Define $H^{\prime}(T)=\bigcup_{x} \mathbb{T}^{\prime}(x)$; all of these tubes intersect $T_{0}$. We have that

$$
\left.\left|H^{\prime}(T)\right| \geq(w \lambda t) / K\right)^{O(1)} \delta^{-2}
$$

Furthermore, for each plane $\Pi$ containing the line coaxial with $T_{0}$; for each point $z \in T_{0}$; and for each $\delta \leq \rho \leq 1$, we have

$$
\left|\left\{T \in H^{\prime}\left(T_{0}\right): T \subset N_{10 \delta}(\Pi), \operatorname{dist}\left(z, T \cap T_{0}\right) \leq \rho\right\}\right| \lesssim(K /(w \lambda t))^{O(1)}(\rho / \delta) .
$$

Wolff's hairbrush argument from [22] says that the union of any set of tubes intersecting $T_{0}$ that satisfy (52) and (53) must have volume $\gtrsim(w \lambda t / K)^{O(1)} \delta$. Thus

$$
\left|\bigcup_{T \in H\left(T_{0}\right)} Y(T)\right| \geq\left|\bigcup_{T \in H^{\prime}\left(T_{0}\right)} Y(T)\right| \gtrsim(w \lambda t / K)^{O(1)} \delta .
$$

The following lemma gives sufficient conditions for a semi-algebraic subset of a hypersurface in $\mathbb{R}^{4}$ to be large (specifically, for it to have $\delta$-covering number roughly $\left.\delta^{-3}\right)$. In short, if a semi-algebraic subset of a hypersurface contains at least one line whose hairbrush contains many cones, then the set must be large.

Lemma 7.6. Let $\delta, c, s, w, \kappa$ be positive real numbers. Let $P$ be a polynomial of degree at most D. Let

$$
Z_{2} \subset Z_{1} \subset\left\{x \in Z(P) \cap B(0,1), 1 \leq|\nabla P(x)| \leq 2,\|I I(x)\|_{\infty} \geq \kappa\right\} .
$$

Let $\emptyset \neq \Sigma_{2} \subset \Sigma_{1}$ with $\Sigma_{i} \subset \Sigma_{\delta, c}\left(Z_{i}\right)$ for $i=1,2$. Let $\Gamma_{2} \subset \Gamma_{1}$ with $\Gamma_{i} \subset \Gamma\left(N_{\delta}\left(Z_{i}\right), \Sigma_{i}\right)$ for $i=1,2$. Suppose that the sets $Z_{i}, \Sigma_{i}$, and $\Gamma_{i}, i=1,2$ are semi-algebraic of complexity at most $E$. For each $i=1,2$, let $\Phi_{i} \subset Z_{i} \times S^{3}$ be associated to $\Gamma_{i}$, in the sense of Definition 5.4. Suppose that

$$
\begin{aligned}
& Z_{2} \subset 1-\operatorname{SBroad}_{s}\left(\Phi_{1}\right) \cap(2,2)-\operatorname{Broad}_{w}\left(\Phi_{1}\right) \\
& \left|\Gamma_{i}(\ell)\right| \geq c \text { for each } \ell \in \Sigma_{i}, i=1,2 \\
& \left|(v \cdot \nabla)^{j} P(z)\right| \leq K \delta, j=1,2, \text { for each } z \in Z_{2} \text { and each } v \in \pi_{S}\left(\Phi_{2}(z)\right) .
\end{aligned}
$$

Then

$$
\mathcal{E}_{\delta}\left(Z_{1}\right){ }_{D, E}(s c w \kappa)^{O(1)} \delta^{-3}
$$


Proof. For $i=1,2$, define $\mathbb{T}_{i}$ to be a maximal $\delta$-separated subset of $\Sigma_{i}$ and for each $T \in \mathbb{T}_{i}$, define $Y_{i}(T)$ to be the $\delta$-neighborhood of $\Gamma_{i}(T)$. Since $\Sigma_{2}$ is non-empty, there exists a tube $T_{0} \in \mathbb{T}_{2}$. By Lemma 7.4, the tube $T_{0}$ and the pair $(\mathbb{T}, Y)$ satisfy the hypotheses of Lemma 7.5. Applying Lemma 7.5, we conclude that

$$
\left|\bigcup_{T \in \mathbb{T}_{1}: Y_{1}(T) \cap Y_{2}\left(T_{0}\right) \neq \emptyset} Y_{1}(T)\right| \gtrsim(s c w \kappa)^{O(1)} \delta .
$$

But since the above set is contained in $N_{\delta}\left(Z_{1}\right)$, we have $\mathcal{E}_{\delta}\left(Z_{1}\right) \gtrsim(s c w \kappa)^{O(1)} \delta^{-3}$.

\subsection{Lines in broad varieties lie near a quadratic hypersurface}

We are now ready to state the main result of this section.

Proposition 7.1. Let $\delta, s, w, \kappa, t$ be positive real numbers. Let $P$ be a polynomial of degree at most D. Let

$$
Z \subset\left\{x \in Z(P) \cap B(0,1), 1 \leq|\nabla P(x)| \leq 2,\|I I(x)\|_{\infty} \geq \kappa\right\} .
$$

Let $\Sigma \subset \mathcal{L}$ with $\left|\mathcal{E}_{\delta}(\Sigma)\right| \geq L^{-1} \delta^{-3}$ and let $\Gamma \subset \Gamma\left(N_{\delta}(Z), \Sigma\right)$. Suppose that $Z, \Sigma$, and $\Gamma$ are semi-algebraic of complexity at most $E$. Let $\Phi \subset Z \times S^{3}$ be associated to $\Gamma$, in the sense of Definition 5.4. Suppose that

$$
\begin{aligned}
& Z \subset 1-\operatorname{SBroad}_{s}(\Phi) \cap(2,2)-\operatorname{Broad}_{w}(\Phi), \\
& \mathcal{E}_{\delta}(Z) \geq t \delta^{-3} \\
& \left|(v \cdot \nabla)^{j} P(z)\right| \leq K \delta, \quad j=1,2 \quad \text { for each } z \in Z \text { and each } v \in \pi_{S}(\Phi(z)) .
\end{aligned}
$$

Then there is a set $\Sigma^{\prime} \subset \Sigma$ and a quadratic polynomial $Q$ so that

$$
\mathcal{E}_{\delta}\left(\Sigma^{\prime}\right) \gtrsim_{D, E}(s w \kappa t / K L)^{O(1)} \mathcal{E}_{\delta}(\Sigma)
$$

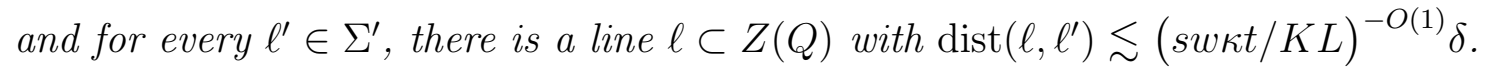

Proof. Since the constants $D$ and $E$ are fixed, all implicit constants may depend on these quantities; i.e. we will write $\lesssim$ instead of $\lesssim_{D, E}$.

For each $\ell \in \Sigma$, let $T_{\ell}=N_{\delta}(\ell) \cap B(0,1)$ and define $Y(T)=N_{\delta}(\Gamma(\ell))$. Let $\mathbb{T}$ be a maximal essentially distinct subset of $\left\{T_{\ell}: \ell \in \Sigma\right\}$. Note that

$$
|\mathbb{T}| \sim \mathcal{E}_{\delta}(\Sigma) \geq L^{-1} \delta^{-3}
$$


By (59), we have that for each $x \in \bigcup_{\mathbb{T}} Y(T)$,

$$
|\mathbb{T}(x)| \gtrsim s \delta^{-1} \text {. }
$$

By (71), (59), 61), and Lemma 7.4, we have that $v(\mathbb{T}(x))$ is contained in the $\lesssim$ $\delta(K / w \kappa)^{O(1)}$-neighborhood of the quadratic cone $\mathcal{C}_{x}$ of $P$ at $x$. By $(59)$, this cone is $\gtrsim w s^{O(1)}$-non-degenerate. Thus there exists a constant $A \lesssim(K / w \kappa)^{O(1)}$ so that for every $x \in Z$, we have that $v(\mathbb{T}(x))$ is contained in the $A \delta$-neighborhood of $\mathcal{C}_{x}$. In particular,

$$
|\mathbb{T}(x)| \lesssim A^{O(1)} \delta^{-1}
$$

Since $t \delta \lesssim\left|N_{\delta}(Z)\right| \lesssim \delta$ (the lower bound comes from 60 and the upper bound comes from the fact that $\left.\left|N_{\delta}(Z)\right| \lesssim \delta\right)$, we have

$$
s t \lesssim \int_{N_{\delta}(Z)} \sum_{T \in \mathbb{T}} \chi_{T}(x) d x \lesssim A^{O(1)}
$$

and thus by $(63)$ and $(66)$,

$$
L^{-1} \delta^{-3} \lesssim|\mathbb{T}| \lesssim A^{O(1)} \delta^{-3}
$$

By pigeonholing, we can select a point $x_{0} \in \bigcup Y(T)$ with

$$
\sum_{T \in \mathbb{T}\left(x_{0}\right)}|Y(T)| \gtrsim(s t)^{O(1)} \delta^{2} .
$$

For each point $x \in \bigcup_{\mathbb{T}\left(x_{0}\right)} Y(T)$, define

$$
N(x)=\left|\left\{\mathbb{T}(x) \cap \bigcup_{T \in \mathbb{T}\left(x_{0}\right)} H(T)\right\}\right| .
$$

$N(x)$ is an integer satisfying $0 \leq N(x) \lesssim A^{O(1)} \delta^{-1}$. For each $T \in \mathbb{T} \backslash \mathbb{T}\left(x_{0}\right)$, define a new shading

$$
Y^{\prime}(T)=\left\{x \in Y(T): N(x) \gtrsim(s t)^{C} \delta^{-1}\right\} .
$$

If the constant $C$ is chosen sufficiently large, then

$$
\sum_{T \in \mathbb{T} \backslash \mathbb{T}\left(x_{0}\right)}\left|Y^{\prime}(T)\right| \geq \frac{1}{2} \sum_{T \in \mathbb{T}}|Y(T)| .
$$

Thus by pigeonholing, we can select a set $\mathbb{T}^{\prime} \subset \mathbb{T}$ so that $\left|\mathbb{T}^{\prime}\right| \gtrsim(s t / L)^{O(1)}|\mathbb{T}|$ and $\left|Y^{\prime}(T)\right| \gtrsim(s t / L)^{C}|T|$ for all $T \in \mathbb{T}^{\prime}$. Select a point $x_{1}$ with $\operatorname{dist}\left(x_{0}, x_{1}\right) \gtrsim(s t / L)^{O(1)}$ and

$$
\left|\mathbb{T}^{\prime}\left(x_{1}\right)\right| \gtrsim(s t / L)^{O(1)} \delta^{-1}
$$


For this value of $x_{1}$, if we select the constant $C \lesssim 1$ sufficiently large then there are $\gtrsim(s t / L)^{O(1)} \delta^{-3}$ tubes $T \in \mathbb{T}$ that satisfy $|Y(T)| \geq(s t / L)^{C}|T|$ and

$\exists T_{0} \in \mathbb{T}\left(x_{0}\right), T_{1} \in \mathbb{T}^{\prime}\left(x_{1}\right): T \cap T_{i} \neq \emptyset, i=1,2, \operatorname{dist}\left(T \cap T_{0}, T \cap T_{1}\right) \geq C^{-1}(s t / L)^{C}$.

Call this set of tubes $\mathbb{T}^{\prime \prime}$. Select a tube $T_{0} \in \mathbb{T}^{\prime \prime}$ with $\left|H\left(T_{0}\right) \cap \mathbb{T}^{\prime \prime}\right| \gtrsim(s t / L)^{O(1)} \delta^{-2}$. Let $\mathcal{C}_{x_{0}}$ be the quadratic cone associated to $x_{0}$ and let $\tilde{\mathcal{C}}_{x_{0}}=x_{0}+\operatorname{span}\left(\mathcal{C}_{x_{0}}\right)$. Define $\tilde{\mathcal{C}}_{x_{1}}$ similarly, with $x_{1}$ in place of $x_{0}$.

Equation (68) implies that

$$
\left|N_{A \delta}\left(\tilde{\mathcal{C}}_{x_{0}}\right) \cap N_{A \delta}\left(\tilde{\mathcal{C}}_{x_{1}}\right)\right| \gtrsim(s t / L)^{O(1)} \delta^{3} .
$$

Since the cones $\tilde{\mathcal{C}}_{x_{0}}$ are $\gtrsim A$ non-degenerate and their vertices are $\gtrsim(s c)^{C}$-separated, the set $N_{A \delta}\left(\tilde{\mathcal{C}}_{x_{0}}\right) \cap N_{A \delta}\left(\tilde{\mathcal{C}}_{x_{1}}\right)$ is contained in the $\lesssim(A L / s t)^{O(1)} \delta$-neighborhood of a curve. However, it need not be the case that the cones $\tilde{\mathcal{C}}_{x_{0}}$ and $\tilde{\mathcal{C}}_{x_{1}}$ themselves intersect. To overcome this annoying technicality, we will replace $\tilde{\mathcal{C}}_{x_{1}}$ by a different cone that is comparable to $\tilde{\mathcal{C}}_{x_{1}}$ but which does intersect $\tilde{\mathcal{C}}_{x_{0}}$ in a curve. We will call this cone $\tilde{\mathcal{C}}^{*}$; we will describe its construction in the next paragraph.

By our choice of $x_{1}$, there exist three points $p_{1}, p_{2}, p_{3} \in \tilde{\mathcal{C}}_{x_{0}} \cap N_{A \delta}\left(\tilde{\mathcal{C}}_{x_{1}}\right)$ so that all $3 \times 3$ minors of $\left[p_{1}-x_{1}, p_{2}-x_{1}, p_{3}-x_{3}\right]$ have magnitude $\gtrsim(s t / A L)^{O(1)}$. Let $H$ be the hyperplane passing through $x_{1}, p_{1}, p_{2}, p_{3}$ (our condition on the minors of $\left[p_{1}-x_{1}, p_{2}-x_{1}, p_{3}-x_{3}\right]$ ensures that this hyperplane is "well conditioned" in the sense that a small perturbation to one of the points $p_{1}, p_{2}$, or $p_{3}$ will only cause a small change in the choice of hyperplane). Since $p_{1}, p_{2}, p_{3} \in N_{A \delta}\left(\tilde{\mathcal{C}}_{x_{1}}\right)$, and $\tilde{\mathcal{C}}_{x_{1}} \subset T_{x_{1}}(Z)$, the condition on the minors of $\left[p_{1}-x_{1}, p_{2}-x_{1}, p_{3}-x_{3}\right]$ implies that

$$
\angle\left(H, T_{x_{1}}(Z)\right) \lesssim(L / s t)^{O(1)} A \delta .
$$

Define $\mathcal{C}^{*}=H \cap Z\left(P_{1}\right)$, where $P_{1}$ is the homogeneous polynomial of degree 2 arising from the Taylor expansion of $P$ around $x_{1}$. Since $H$ and $Z\left(P_{1}\right)$ intersect $\geq \kappa$ transversely (i.e. the tangent plane of $Z\left(P_{1}\right)$ and of $H$ make an angle $\geq \kappa$ at every point of intersection), (69) implies that $\tilde{\mathcal{C}}_{x_{1}}$ and $\mathcal{C}^{*}$ are comparable in the sense that

$$
B(0,1) \cap \tilde{\mathcal{C}}_{x_{1}} \subset N_{(A L /(s t \kappa))^{O(1)} \delta}\left(\mathcal{C}^{*}\right), \quad \text { and } \quad B(0,1) \cap \mathcal{C}^{*} \subset N_{(A L /(s t \kappa))^{O(1)} \delta}\left(\tilde{\mathcal{C}}_{x_{1}}\right) .
$$

We also have that $\tilde{\mathcal{C}}_{x_{0}} \cap \mathcal{C}^{*}$ is a degree-two curve lying in the plane $T_{x_{0}} Z \cap H$.

Let $\ell$ be a line with $\ell \cap B(0,1) \subset T_{0}$ so that $\ell$ intersects $\tilde{\mathcal{C}}_{x_{0}}$ and $\mathcal{C}^{*}$ at points that are $\gtrsim(s t \kappa / A L)^{O(1)}$ separated. Observe that the cones $\tilde{\mathcal{C}}_{x_{0}}$ and $\mathcal{C}^{*}$ intersect in a one-dimensional degree-two curve, and the line $\ell$ intersects each of $\tilde{\mathcal{C}}_{x_{0}}$ and $\mathcal{C}^{*}$ at distinct points that are not on this curve. 
We can now use the "14 point" argument from [14] to find a monic polynomial $Q$ that vanishes on $\tilde{\mathcal{C}}_{x_{0}}, \mathcal{C}^{*}$, and $\ell$. In brief, select 5 points $p_{1}, \ldots, p_{5} \in \tilde{\mathcal{C}}_{x_{0}} \cap \mathcal{C}^{*}$; any polynomial of degree $\leq 2$ that vanishes on $p_{1}, \ldots, p_{5}$ must vanish on the degree-two plane curve $\tilde{\mathcal{C}}_{x_{0}} \cap \mathcal{C}^{*}$. Let $p_{6}, p_{7}$ be the points of intersection of $\tilde{\mathcal{C}}_{x_{0}} \cap \ell$ and $\mathcal{C}^{*} \cap \ell$, and let $p_{8}$ be another point on $\ell$; any polynomial of degree $\leq 2$ that vanishes on $p_{6}, p_{7}, p_{8}$ must vanish on $\ell$. Let $p_{9}=x_{0}$ and let $p_{10}=x_{1}$. Let $p_{11}$ and $p_{12}$ be two points on $\tilde{\mathcal{C}}_{x_{0}}$, and let $p_{13}$ and $p_{14}$ be two points on $\mathcal{C}^{*}$. See Figure 4 . Let $Q$ be a polynomial of degree $\leq 2$ that vanishes on $p_{1}, \ldots, p_{14}$. We can choose $Q$ so that its largest coefficient has magnitude 1. $Q$ will be the output from this proposition. The remainder of the proof is devoted to finding the set $\Sigma^{\prime}$ so that $Q$ and $\Sigma^{\prime}$ satisfy the conclusions of the proposition.

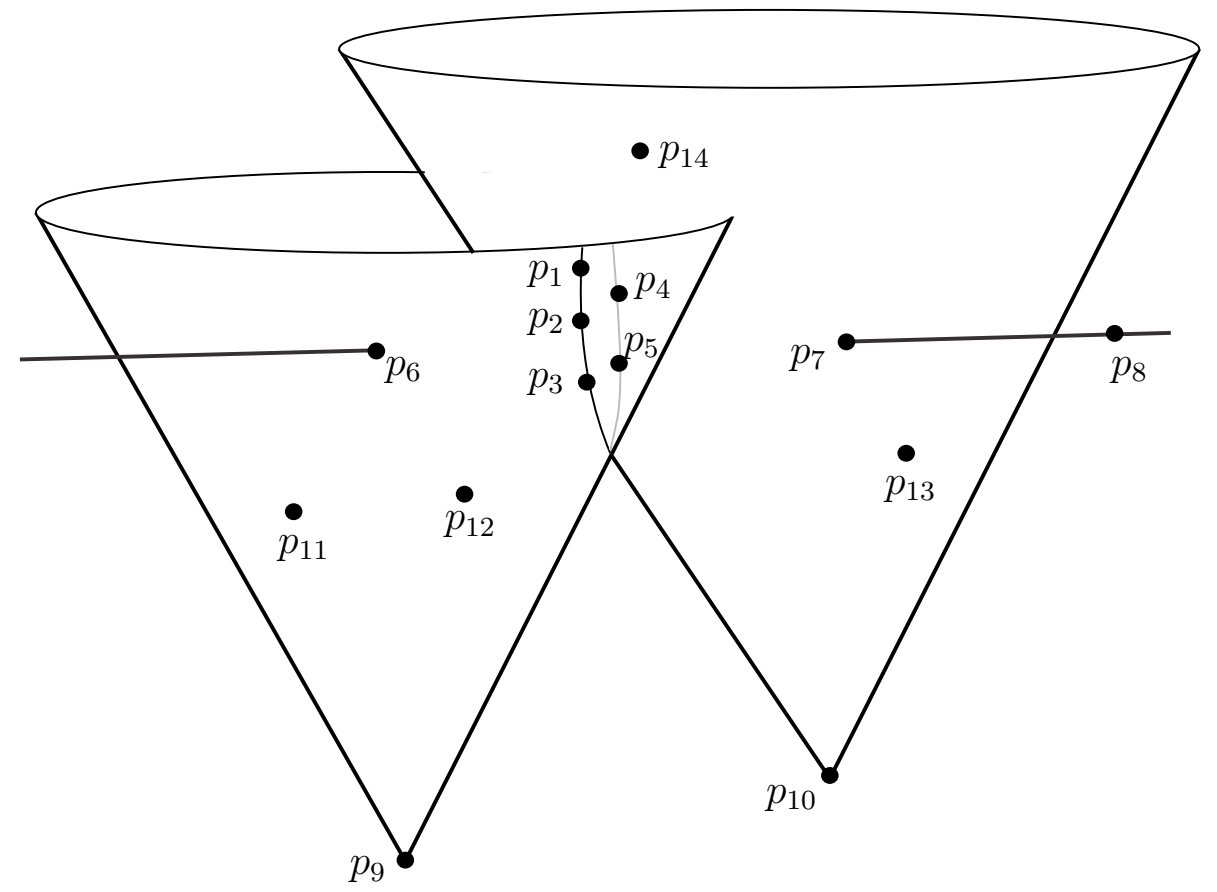

Figure 4: The cones $\tilde{\mathcal{C}}_{x_{0}}$ (left), $\mathcal{C}^{*}$ (right), the line $\ell$, and the 14 points $p_{1}, \ldots, p_{14}$.

Let $\ell_{1}$ be the line passing through $p_{9}$ and $p_{11}$; this is a line in $\tilde{\mathcal{C}}_{x_{0}}$ passing through the vertex $p_{9}=x_{0}$, so it intersects the curve $\tilde{\mathcal{C}}_{x_{0}} \cap \mathcal{C}^{*}$ at some point $x$. Since $Q$ vanishes at the three collinear points $p_{9}, p_{11}$, and $x, Q$ must vanish on the entire line $\ell_{1}$. Similarly, $Q$ vanishes on the line $\ell_{2}$ passing through $p_{9}$ and $p_{12}$, and the line $\ell_{3}$ passing through $p_{9}$ and $p_{6}$. Thus $Q$ vanishes on the five-dimensional (reducible) curve $\tilde{\mathcal{C}}_{x_{0}} \cap \mathcal{C}^{*} \cup \ell_{1} \cup \ell_{2} \cup \ell_{3}$. Since $Q$ has degree at most 2 and $\tilde{\mathcal{C}}_{x_{0}}$ has degree at 
most 2 , we conclude that $Q$ vanishes on $\tilde{\mathcal{C}}_{x_{0}}$. An identical argument shows that $Q$ vanishes on $\mathcal{C}^{*}$.

Recall that for each $T \in H\left(T_{0}\right) \cap \mathbb{T}^{\prime \prime}$, we have that $Z(Q)$ vanishes on $\gtrsim(s t \kappa / A L)^{O(1)} \delta^{-1}$ distinct $\delta$-separated points on $T$. Since $Q$ is monic and has degree 2 , we have

$$
|Q(x)| \lesssim(A L / s t \kappa)^{O(1)} \delta \quad \text { for all } x \in T .
$$

By the definition of $\mathbb{T}^{\prime \prime}$, we have

$$
\sum_{T \in H\left(T_{0}\right) \cap \mathbb{T}^{\prime \prime}}\left|H_{Y}(T)\right| \gtrsim(s t \kappa / A L)^{O(1)} \delta^{-4} .
$$

Thus there exists a set $\mathbb{T}^{\prime \prime \prime}$ with $\left|\mathbb{T}^{\prime \prime \prime}\right| \gtrsim(s t \kappa / A L)^{O(1)} \delta^{-3}$ and

$$
\left|Y\left(T^{\prime}\right) \cap \bigcup_{T \in H\left(T_{0}\right)} Y(T)\right| \gtrsim(s t \kappa / A L)^{O(1)}\left|T^{\prime}\right| \quad \text { for every } T^{\prime} \in \mathbb{T}^{\prime \prime \prime} .
$$

Since $Q$ is monic and $Z(Q) \cap B(0,1) \neq \emptyset$, we can assume that at least one nonconstant term of $Q$ has size $\sim 1$. We can also assume that at least one degree-two term of $Q$ has magnitude $\gtrsim(\kappa s t / A L)^{O(1)}$; if this were not the case, then $Z(Q) \cap B(0,1)$ would be contained in the $\sim(\kappa s t / A L)^{O(1)}$-neighborhood of a hyperplane $H$, and thus

$$
\left|N_{\delta}(Z) \cap N_{(\kappa s t / A L)^{O(1)}}(H)\right| \geq \bigcup_{T \in H\left(T_{0}\right)} Y(T) \gtrsim(\kappa s t / A L)^{O(1)} \delta,
$$

but this would contradict the estimate

$$
\left|N_{\delta}(Z) \cap N_{(\kappa s t / A L)^{O(1)}}(H)\right| \lesssim \delta^{3 / 2}(\kappa s t / A L)^{-O(1)}
$$

coming from Lemma 4.3 . Thus at least one degree-two term of $Q$ must have magnitude $\gtrsim(\kappa s t / A L)^{O(1)}$. Next, the set

$$
\left\{x \in B(0,1):|\nabla Q(x)| \leq(\kappa s t / A L)^{O(1)}\right\}
$$

is contained in the $(\kappa s t / A L)^{O(1)}$-neighborhood of a hyperplane $H^{\prime}$. By the same argument as above, we can choose a refinement $\mathbb{T}^{(\mathrm{iv})} \subset \mathbb{T}^{\prime \prime \prime}$ with

$$
\left|\mathbb{T}^{(\mathrm{iv})}\right| \gtrsim(\kappa s t / A L)^{O(1)} \delta^{-3}
$$

and a shading $Y^{(\mathrm{iv})}(T)$ so that $|\nabla Q(x)| \gtrsim(\kappa s c / A)^{O(1)}$ for all $x \in Y^{(\mathrm{iv})}(T)$ and all $T \in \mathbb{T}^{(\mathrm{iv})}$. 
Again by pigeonholing, we can refine $Y^{(\mathrm{iv})}$ to get a shading $Y^{(\mathrm{v})}$ so that $\left|\mathbb{T}_{Y^{(\mathrm{v})}}(x)\right| \gtrsim$ $(\kappa s t / A L)^{O(1)} \delta^{-1}$ for all $x \in \bigcup_{T \in \mathbb{T}^{(i v)}} Y^{(\mathrm{v})}(T)$. Now fix a point $x \in \bigcup_{T \in \mathbb{T}^{(i v)}} Y^{(\mathrm{v})}(T)$. We will show that $T_{x}(Z(Q)) \cap Z(Q)$ is a $\zeta$-non-degenerate cone, where $\zeta=(A L /(s t \kappa))^{O(1)}$. Indeed, $v\left(\mathbb{T}^{(\mathrm{iv})}(x)\right)$ is contained in the $A \delta$-neighborhood of the $w$-non-degenerate cone $\mathcal{C}_{x}$, and $\left|\mathbb{T}^{(\mathrm{iv})}(x)\right| \gtrsim(s t \kappa / A L)^{O(1)} \delta^{-1}$. At most $(\zeta / w)^{1 / 2} A \delta \delta$-separated vectors can be contained in the intersection of $N_{A}\left(\mathcal{C}_{x}\right)$ with the $\zeta$-neighborhood of a plane. We conclude that the cone $T_{x}(Z(Q)) \cap Z(Q)$ is $\zeta$-non-degenerate for some $\zeta=(s c t \kappa / A)^{O(1)}$.

We conclude that if $T \in \mathbb{T}^{(\mathrm{iv})}$ and $x \in Y^{(\mathrm{v})}(T)$, then $v(T)$ makes an angle $\lesssim$ $(A L /(s t \kappa))^{O(1)} \delta$ with the quadratic cone $T_{x}(Z(Q)) \cap Z(Q)$ of $Q$ at $x$. However, since $Q$ is degree-two, if $v$ is a vector contained in the quadratic cone of $Q$ at $x$, then the line $\{x+v t: t \in \mathbb{R}\}$ is contained in $Z(Q)$. Thus if $T \in \mathbb{T}^{(\mathrm{iv})}$ with $Y^{(\mathrm{v})}(T) \neq \emptyset$, then there is a line $\ell$ contained in $Z(Q)$ with $\left.\ell \cap B(0,1) \subset N_{(A L /(s t \kappa))}\right)_{(1) \delta}(T)$.

By (67) and (70), we have that

$$
\begin{aligned}
\left|\mathbb{T}^{(\mathrm{iv})}\right| & \geq(s t \kappa / A L)^{O(1)}|\mathbb{T}| \\
& \gtrsim(s w t \kappa / K L)^{O(1)}|\mathbb{T}| \\
& \gtrsim(s w t \kappa / K L)^{O(1)} \mathcal{E}_{\delta}(\Sigma) .
\end{aligned}
$$

Thus there is a set $\Sigma^{\prime} \subset \Sigma$ (note that $\Sigma^{\prime}$ need not be semi-algebraic) with

$$
\mathcal{E}_{\delta}\left(\Sigma^{\prime}\right) \gtrsim(s w t \kappa / K L)^{O(1)} \mathcal{E}_{\delta}(\Sigma)
$$

so that for all $\ell^{\prime} \in \Sigma^{\prime}$ there is a line $\ell$ contained in $Z(Q)$ with

$$
\operatorname{dist}\left(\ell, \ell^{\prime}\right) \lesssim(s w t \kappa / K L)^{O(1)} \delta .
$$

\section{Proof of Theorem 1.2}

The following result allows us to separate the lines in $\Sigma_{\delta}(Z)$ into two sets - those that can be covered by a small number of one and two-dimensional rectangular prisms, and those that are amenable to Proposition 7.1.

Proposition 8.1. Let $\delta, s, u, c, \kappa$ be positive real numbers. Let $P$ be a polynomial of degree at most $D$. Let

$$
Z \subset\left\{x \in Z(P) \cap B(0,1), 1 \leq|\nabla P(x)| \leq 2,\|I I(x)\|_{\infty} \geq \kappa\right\} .
$$


Let $\Sigma \subset \Sigma_{\delta, c}(Z)$ and $\Gamma \subset \Gamma\left(N_{\delta}(Z), \Sigma\right)$. Suppose that $Z, \Sigma$, and $\Gamma$ are semi-algebraic of complexity at most $E$. Let $\Phi \subset Z \times S^{3}$ be associated to $\Gamma$, in the sense of Definition 5.4. Suppose that

$|\Gamma(\ell)| \geq c, \quad$ for all $\ell \in \Sigma$,

$\left|(v \cdot \nabla)^{j} P(z)\right| \leq K \delta, \quad j=1,2 \quad$ for each $z \in Z$ and each $v \in \pi_{S}(\Phi(z))$.

Then there is a number $w \gtrsim_{D, E}(u s \kappa c)^{O(1)}$ and sets $\Sigma^{\prime}, \Sigma^{\prime \prime}, \Sigma^{\prime \prime \prime}, Z^{\prime \prime \prime}$ and $\Gamma^{\prime \prime \prime}$ with $\Sigma=\Sigma^{\prime} \cup \Sigma^{\prime \prime} \cup \Sigma^{\prime \prime \prime}, \Sigma^{\prime \prime \prime} \subset \Sigma_{\delta, c / 27}\left(Z^{\prime \prime \prime}\right)$, and $\Gamma^{\prime \prime \prime} \subset \Gamma\left(N_{\delta}\left(Z^{\prime \prime \prime}\right), \Sigma^{\prime \prime \prime}\right)$, so that

- The lines in $\Sigma^{\prime}$ can be covered by $O_{D, E}\left(c^{-O(1)} s^{-2}\right)$ rectangular prisms of dimensions $2 \times s \times s \times s$.

- The lines in $\Sigma^{\prime \prime}$ can be covered by $O_{D, E}\left((s c)^{-O(1)} u^{-1}\right)$ rectangular prisms of dimensions $2 \times 2 \times u \times u$.

- $\Sigma^{\prime \prime \prime}, Z^{\prime \prime \prime}$ and $\Gamma^{\prime \prime \prime}$ are semi-algebraic of complexity $O_{D, E}(1)$. We have

$$
\left|N_{\delta}\left(\Sigma^{\prime \prime \prime}\right)\right| \lesssim_{D}(K /(u s \kappa c))^{O(1)} \delta^{-3} .
$$

Finally, let $\Phi^{\prime \prime \prime}$ be the set associated to $\Gamma^{\prime \prime \prime}$. Then

$$
\begin{aligned}
& Z^{\prime \prime \prime} \subset 1-\operatorname{SBroad}_{s}\left(\Phi^{\prime \prime \prime}\right) \cap(2,2)-\operatorname{Broad}_{w}\left(\Phi^{\prime \prime \prime}\right), \\
& \mathcal{E}_{\delta}\left(Z^{\prime \prime \prime}\right) \gtrsim(\operatorname{scu\kappa })^{O(1)} \delta^{-3} .
\end{aligned}
$$

Proof. Let $w \gtrsim_{D, E}(\text { uskc })^{O(1)}$ be the constant from Proposition 6.2 associated to the values $u, s, \kappa, c, D, E$. Define $\Sigma_{1}=\Sigma, \Gamma_{1}=\Gamma$, and $\Phi_{1}=\Phi$. For each $i=1,2,3$, inductively define

$$
\begin{aligned}
& \Phi_{i}^{\prime}=\left\{(z, v) \in \Phi_{i}: z \notin 1-\operatorname{SBroad}_{s}\left(\Phi_{i}\right)\right\}, \\
& \Phi_{i}^{\prime \prime}=\left\{(z, v) \in \Phi_{i}: z \in(2,2)-\operatorname{Narrow}_{w}\left(\Phi_{i}\right)\right\}, \\
& \Phi_{i}^{\prime \prime \prime}=\Phi_{i} \backslash\left(\Phi_{i}^{\prime} \cup \Phi_{i}^{\prime \prime}\right) .
\end{aligned}
$$

Let $\Gamma_{i}^{\prime}, \Gamma_{i}^{\prime \prime}$, and $\Gamma_{i}^{\prime \prime \prime}$ be the pre-images of $\Phi_{i}^{\prime}, \Phi_{i}^{\prime \prime}$, and $\Phi_{i}^{\prime \prime \prime}$ (respectively) under the map $\Gamma_{i} \rightarrow \Phi_{i}$. Define

$$
\begin{aligned}
\Sigma_{i}^{\prime} & =\left\{\ell \in \Sigma_{i}:\left|\Gamma_{i}^{\prime}(\ell)\right| \geq 3^{-i} c\right\} \\
\Sigma_{i}^{\prime \prime} & =\left\{\ell \in \Sigma_{i}:\left|\Gamma_{i}^{\prime \prime}(\ell)\right| \geq 3^{-i} c\right\} \\
\Sigma_{i}^{\prime \prime \prime} & =\left\{\ell \in \Sigma_{i}:\left|\Gamma_{i}^{\prime \prime \prime}(\ell)\right| \geq 3^{-i} c\right\}
\end{aligned}
$$


so $\Sigma_{i} \subset \Sigma_{i}^{\prime} \cup \Sigma_{i}^{\prime \prime} \cup \Sigma_{i}^{\prime \prime \prime}$

By Proposition 6.1, the lines in $\Sigma_{i}^{\prime}$ can be covered by $O_{D, E}\left(c^{-O(1)} s^{-2}\right)$ rectangular prisms of dimensions $2 \times s \times s \times s$; these lines will be placed in $\Sigma^{\prime}$. By Proposition 6.2 , the lines in $\Sigma_{i}^{\prime \prime}$ can be partitioned into two sets; the first set can be covered by $O_{D, E}\left(c^{-O(1)} s^{-2}\right)$ rectangular prisms of dimensions $2 \times s \times s \times s$ and the second set can be covered by $O_{D, E}\left((c s)^{-O(1)} u^{-1}\right)$ rectangular prisms of dimensions $2 \times 2 \times u \times u$. We will place these lines into $\Sigma^{\prime}$ and $\Sigma^{\prime \prime}$, respectively.

Define

$$
\Sigma_{i+1}=\Sigma_{i}^{\prime \prime \prime}
$$

and define

$$
\Gamma_{i+1}=\Gamma_{i}^{\prime \prime \prime}
$$

Define $Z_{i+1}$ to be the image of $\Phi_{i}$ under the map $(z, v) \mapsto z$. Then we have

- $Z_{i+1} \subset Z_{i}$.

- $\Sigma_{i+1} \subset \Sigma_{\delta, 3^{-i} c}\left(Z_{i}\right)$.

- $\Sigma_{i+1} \subset \Sigma_{i}$.

- $\Gamma_{i+1} \subset \Gamma_{i}$.

$$
\begin{gathered}
Z_{i+1} \subset 1-\operatorname{SBroad}_{s}\left(\Phi_{i}\right) \cap(2,2)-\operatorname{Broad}_{w}\left(\Phi_{i}\right) . \\
\left|\Gamma_{i}(\ell)\right| \geq 3^{-i} c \geq c / 27 .
\end{gathered}
$$

- By 73),

$$
\left|(v \cdot \nabla)^{j} P(z)\right| \leq K \delta, j=1,2, \text { for each } z \in Z_{i} \text { and each } v \in \pi_{S}\left(\Phi_{i}(z)\right) .
$$

If $\Sigma_{3}=\emptyset$ then define $\Sigma^{\prime \prime \prime}=\emptyset, Z^{\prime \prime \prime}=\emptyset$ and $\Gamma^{\prime \prime \prime}=\emptyset$ and we are done. If not, define $Z^{\prime \prime \prime}=Z_{2}, \Sigma^{\prime \prime \prime}=\Sigma_{1}$, and $\Gamma^{\prime \prime \prime}=\Gamma_{2}$. (75) follows from (77). Applying Lemma 7.6 to the sets $Z_{3} \subset Z_{2} ; \Sigma_{3} \subset \Sigma_{2} ; \Gamma_{3} \subset \Gamma_{2}$ (equations (77), (78) and (79) guarantee that the hypotheses of Lemma 7.6 are met), we conclude that

$$
\mathcal{E}_{\delta}\left(Z^{\prime \prime \prime}\right)=\mathcal{E}_{\delta}\left(Z_{2}\right) \gtrsim(s c w \kappa)^{O(1)} \delta^{-3},
$$

which is (76).

Finally, it remains to prove (74). But this follows from the observation that $\left|N_{\delta}(Z)\right| \lesssim_{D} \delta^{-3}$, and that for each $z \in Z$,

$$
N_{\delta}\left(\Phi^{\prime \prime \prime}\left(N_{\delta}(z)\right) \lesssim_{D, E}(K /(u s \kappa c))^{O(1)} \delta^{-1}\right.
$$


We are now ready to prove Theorem 1.2 . First, we will state a slightly more technical version that will be useful for applications.

Theorem 1.2, technical version. Let $P \in \mathbb{R}\left[x_{1}, x_{2}, x_{3}, x_{4}\right]$ be a polynomial of degree $D$, and let $Z=Z(P) \cap B(0,1)$. Let $\delta, \kappa, u, s \in(0,1)$ be numbers satisfying $0<\delta<u<s<1$ and $\delta<\kappa<1$ (if these conditions are not satisfied the theorem is still true, but it has no content).

Define

$$
\Sigma=\left\{\ell \in \mathcal{L}:\left|\ell \cap N_{\delta}(Z)\right| \geq 1\right\},
$$

and let $\Sigma^{\prime} \subset \Sigma$ be a semi-algebraic set of complexity at most $E$. Then we can write $\Sigma^{\prime}=\Sigma_{1} \cup \Sigma_{2} \cup \Sigma_{3} \cup \Sigma_{4}$, where

- There is a collection of $O_{D, E}\left(|\log \delta|^{O(1)} s^{-2}\right)$ rectangular prisms of dimensions $2 \times s \times s \times s$ so that every line from $\Sigma_{1}$ is covered by one of these prisms.

- There is a collection of $O_{D, E}\left((|\log \delta| / s)^{O(1)} u^{-1}\right)$ rectangular prisms of dimensions $2 \times 2 \times u \times u$ so that every line from $\Sigma_{2}$ is covered by one of these prisms.

- There is a collection of $O_{D, E}\left(|\log \delta|^{O(1)}\right)$ rectangular prisms of dimensions $2 \times$ $2 \times 2 \times \kappa$ so that every line in $\Sigma_{3}$ is covered by one of these prisms.

- There is a set $\Sigma_{4}^{\prime} \subset \Sigma_{4}$ with

$$
\mathcal{E}_{\delta}\left(\Sigma_{4}^{\prime}\right) \gtrsim_{D, E}(u s \kappa /|\log \delta|)^{O(1)} \mathcal{E}_{\delta}\left(\Sigma_{4}\right)
$$

and a quadratic hypersurface $Q$ so that for every line $\ell^{\prime} \in \Sigma_{4}^{\prime}$, there is a line $\ell$ contained in $Z(Q)$ with

$$
\operatorname{dist}\left(\ell, \ell^{\prime}\right) \lesssim_{D}(|\log \delta| /(u s \kappa))^{O(1)} \delta
$$

Proof. Apply Proposition 3.1 to $\Sigma$ and $P$. Let $P_{1}, \ldots, P_{b}, \Sigma_{1}, \ldots, \Sigma_{b}$, and $\Gamma_{1}, \ldots, \Gamma_{b}$, $b=O_{D, E}(|\log \delta|)$ be the output from the proposition. By Item 5 from Proposition 3.1. there exists a number $c \gtrsim_{D}|\log \delta|^{-1}$ so that $\left|\Gamma_{j}(\ell)\right| \geq c$ for each $j=1, \ldots, b$ and each $\ell \in \Sigma_{j}$.

For each index $j$, define

$$
Z_{j}=\left\{x \in Z\left(P_{j}\right) \cap B(0,1): 1 \leq\left|\nabla P_{j}(x)\right| \leq 2\right\} .
$$

By Item 3 from Proposition 3.1, we have that for all $\ell \in \Sigma_{j}$ and all $x \in \Gamma_{j}(\ell)$, $x \in N_{\delta}\left(Z_{j}\right)$. Let $\Phi_{j} \subset Z_{j} \times S^{3}$ be associated to $\Gamma_{j}$, in the sense of Definition 5.4. We have that for each $z \in Z_{j}$ and each $v \in \Pi_{S}\left(\Phi_{j}(z)\right)$,

$$
\left|(v(\ell) \cdot \nabla)^{i} P_{j}(z)\right| \lesssim_{D}|\log \delta|^{2} \delta, i=1,2 .
$$


Define

$$
\begin{aligned}
& Z_{j}^{\prime}=\left\{z \in Z_{j}:\|I I(z)\|_{\infty} \leq \kappa\right\} \\
& Z_{j}^{\prime \prime}=\left\{z \in Z_{j}:\|I I(z)\|_{\infty}>\kappa\right\}
\end{aligned}
$$

Define

$$
\begin{aligned}
& \Phi_{j}^{\prime}=\left\{(z, v) \in \Phi_{j}: z \in Z_{j}^{\prime}\right\}, \\
& \Phi_{j}^{\prime \prime}=\left\{(z, v) \in \Phi_{j}: z \in Z_{j}^{\prime \prime}\right\} .
\end{aligned}
$$

Let $\Gamma_{j}^{\prime}$ and $\Gamma_{j}^{\prime \prime}$ be the pre-images of $\Phi_{j}^{\prime}$ and $\Phi_{j}^{\prime \prime}$, respectively, under the map from $\Phi_{j} \rightarrow \Gamma_{j}$. Define

$$
\begin{aligned}
& \Sigma_{j}^{\prime}=\left\{\ell \in \Sigma_{j}:\left|\Gamma_{j}^{\prime}(\ell)\right| \geq c / 2\right\}, \\
& \Sigma_{j}^{\prime \prime}=\left\{\ell \in \Sigma_{j}:\left|\Gamma_{j}^{\prime \prime}(\ell)\right| \geq c / 2\right\} .
\end{aligned}
$$

Apply Proposition 4.1 to $P_{j}, \Sigma_{j}^{\prime}$ and $\Gamma_{j}^{\prime}$. Define $\Sigma_{j}^{(1)}=\Sigma_{j}^{\prime}$. We conclude that the lines in $\Sigma_{j}^{(1)}$ can be covered by $\lesssim_{D, E}(|\log \delta| / c)^{O(1)} \lesssim_{D, E}|\log \delta|^{O(1)}$ rectangular prisms of dimensions $2 \times 2 \times 2 \times \kappa$.

Apply Proposition 8.1 to $P_{j}, Z_{j}^{\prime \prime}, \Sigma_{j}^{\prime \prime}$, and $\Gamma_{j}^{\prime \prime}$, with the parameters $\delta, s, u, c / 2$, and $\kappa$. We obtain a number $w \gtrsim_{D, E}(u s \kappa c)^{O(1)} \gtrsim_{D, E}(u s \kappa /|\log \delta|)^{O(1)}$; sets of lines $\Sigma_{j}^{(2)}$, $\Sigma_{j}^{(3)}$, and $\Sigma_{j}^{(4)}$; and sets $Z_{j}^{(4)}$ and $\Gamma_{j}^{(4)}$ so that $\Sigma_{j}^{\prime \prime}=\Sigma_{j}^{(2)} \cup \Sigma_{j}^{(3)} \cup \Sigma_{j}^{(4)}$, and

- The lines in $\Sigma_{j}^{(2)}$ can be covered by $O_{D, E}\left(c^{-O(1)} s^{-2}\right)=O_{D, E}\left(|\log \delta|^{O(1)} s^{-2}\right)$ rectangular prisms of dimensions $2 \times s \times s \times s$.

- The lines in $\Sigma_{j}^{(3)}$ can be covered by $O_{D, E}\left((s c)^{-O(1)} u^{-1}\right)=O_{D, E}\left((|\log \delta| / s)^{O(1)} u^{-1}\right)$ rectangular prisms of dimensions $2 \times 2 \times u \times u$.

- $\Sigma_{j}^{(4)}, Z_{j}^{(4)}$ and $\Gamma_{j}^{(4)}$ are semi-algebraic of complexity $O_{D, E}(1)$. If $\Phi_{j}^{(4)}$ is the set associated to $\Gamma_{j}^{(4)}$, then

$$
\begin{aligned}
& Z_{j}^{(4)} \subset 1-\operatorname{SBroad}_{s}\left(\Phi_{j}^{(4)}\right) \cap(2,2)-\operatorname{Broad}_{w}\left(\Phi_{j}^{(4)}\right), \\
& \mathcal{E}_{\delta}\left(Z_{j}^{(4)}\right){ }_{D, E}(\operatorname{scu\kappa })^{O(1)} \delta^{-3} \gtrsim_{D, E}(\operatorname{su\kappa } /|\log \delta|)^{O(1)} \delta^{-3} .
\end{aligned}
$$

The sets $Z_{j}^{(4)}, \Sigma_{j}^{(4)}$, and $\Gamma_{j}^{(4)}$ satisfy the hypotheses of Proposition 7.1. Thus there exists a set $\left(\Sigma_{j}^{(4)}\right)^{\prime} \subset \Sigma_{j}^{(4)}$ and a quadratic polynomial $Q_{j}$ so that

$$
\mathcal{E}_{\delta}\left(\left(\Sigma_{j}^{(4)}\right)^{\prime}\right){ }_{D, E}(s u \kappa /|\log \delta|)^{O(1)} \mathcal{E}_{\delta}\left(\Sigma_{j}^{(4)}\right),
$$


and for every $\ell^{\prime} \in\left(\Sigma_{j}^{(4)}\right)^{\prime}$, there is a line $\ell \subset Z\left(Q_{j}\right)$ with

$$
\operatorname{dist}\left(\ell, \ell^{\prime}\right) \lesssim(s u \kappa /|\log \delta|)^{-O(1)} \delta
$$

For $i=1,2,3,4$, define

$$
\Sigma_{i}=\bigcup_{j=1}^{b} \Sigma_{j}^{(i)} .
$$

Then $\Sigma=\Sigma_{1} \cup \Sigma_{2} \cup \Sigma_{3} \cup \Sigma_{4}$, and the sets $\Sigma_{1}, \Sigma_{2}, \Sigma_{3}$, and $\Sigma_{4}$ satisfy the conclusions of Theorem 1.2 (the set $\Sigma_{4}^{\prime}$ is the set of the form $\left(\Sigma_{j}^{(4)}\right)^{\prime}$ that maximizes $\left.\mathcal{E}_{\delta}\left(\left(\Sigma_{j}^{(4)}\right)^{\prime}\right)\right)$.

\section{Proof of Corollary 1.2}

Corollary 1.2 will be proved by combining induction on scale and re-scaling arguments. First, we will state a variant of Corollary 1.2 that is more amenable to induction.

Proposition 9.1. For each $D, \varepsilon>0$, there exists a constant $C_{D, \varepsilon}$ so that the following holds for all $0<\delta \leq 1$.

Let $P \in \mathbb{R}\left[x_{1}, \ldots, x_{4}\right]$ be a polynomial of degree at most $D$ and let $Z=Z(P) \cap$ $B(0,1)$. Then

$$
\left.\mathcal{E}_{\delta}\left(v\left(\Sigma_{\delta}(Z)\right)\right)\right) \leq C_{D, \varepsilon} \delta^{-2-\varepsilon}
$$

\subsection{Re-scaling arguments}

Lemma 9.1. Fix $D$ and $\varepsilon>0$ and let $\delta>0$. Suppose that Proposition 9.1 holds for all values of $\delta^{\prime}$ with $\delta<\delta^{\prime} \leq 1$, and let $C_{D, \varepsilon}$ be the associated constant. Let $P$ be a polynomial of degree at most $D$, and let $Z=Z(P) \cap B(0,1)$. Let $R$ be a rectangular prism (of arbitrary orientation) that has $1 \leq d \leq 3$ "long" directions and 4-d "short" directions; suppose that $R$ has length 2 in the long directions and length $t$ in the short directions (i.e. inside $B(0,1), R$ is comparable to the $t$-neighborhood of a d-dimensional affine hyperplane). Then

$$
\mathcal{E}_{\delta}\left(v\left(\Sigma_{\delta}(Z \cap R)\right)\right) \leq C \cdot C_{D, \varepsilon} t^{3-d+\varepsilon} \delta^{-2-\varepsilon},
$$

where $C$ is an absolute constant.

Proof. Let $H$ be a $d$-dimensional hyperplane with $N_{t}(H) \cap B(0,1)$ comparable to $R \cap B(0,1)$. After applying a translation, we can assume that $H$ contains the origin. 
First, we can assume $t \leq 1 / 100$, or the theorem is trivial. Second, we can assume that $H$ makes an angle $\leq 1 / 5$ with the vector $e_{1}$, since otherwise $\Sigma_{\delta}\left(Z \cap N_{t}(H)\right)$ is empty. Apply a rotation so that $H$ is the $d$-dimensional hyperplane given by $x_{d+1}=0, \ldots, x_{4}=0$. After applying this rotation, it is still true that every line in $\Sigma_{\delta}\left(Z \cap N_{t}(H)\right)$ makes an angle $\leq 1 / 3$ with the $e_{1}$ direction. Note that the map

$$
\begin{aligned}
\left\{v \in S^{4} \subset \mathbb{R}^{4}: \angle\left(v, e_{1}\right) \leq 1 / 3\right\} & \rightarrow \mathbb{R}^{3}, \\
\left(v_{1}, v_{2}, v_{3}, v_{4}\right) & \mapsto\left(v_{2}, v_{3}, v_{4}\right),
\end{aligned}
$$

is bi-Lipschitz with constant $\sim 1$. In particular, if $\ell, \ell^{\prime} \in \Sigma_{\delta}\left(Z \cap N_{t}(H)\right)$ with $\angle\left(\ell, \ell^{\prime}\right) \geq \delta$, and if $v=v(\ell), v^{\prime}=v\left(\ell^{\prime}\right)$, then $\max \left(\left|v_{2}-v_{2}^{\prime}\right|,\left|v_{3}-v_{3}^{\prime}\right|,\left|v_{4}-v_{4}^{\prime}\right|\right) \gtrsim \delta$. Note as well that if $\ell \in \Sigma_{\delta}\left(Z \cap N_{t}(H)\right)$ and if $v=v(\ell)$, then $\left|v_{d+1}\right|, \ldots,\left|v_{4}\right| \lesssim t$.

Let $f: \mathbb{R}^{4} \rightarrow \mathbb{R}^{4}$ be the linear map that dilates $x_{d+1}, \ldots, x_{4}$ by a factor of $1 / t$ and leaves $x_{1}, \ldots, x_{d}$ unchanged. Observe that if $\ell \in \Sigma_{\delta}\left(Z \cap N_{t}(H)\right)$, and if $v=v(\ell), \tilde{v}=$ $v(f(\ell))$, then $v_{i} \sim \tilde{v}_{i} / t$ for $i=d+1, \ldots, 4$, and $v_{i} \sim \tilde{v}_{i}$ for $i=2, \ldots, d$. Thus if $\ell, \ell^{\prime} \in \Sigma_{\delta}\left(Z \cap N_{t}(H)\right)$ with $\angle\left(\ell, \ell^{\prime}\right) \geq \delta$, and if $\tilde{v}=v(f(\ell))$ and $\tilde{v}^{\prime}=v\left(f\left(\ell^{\prime}\right)\right)$, then

$$
\max \left(\left|\tilde{v}_{2}-\tilde{v}_{2}^{\prime}\right|, \ldots,\left|\tilde{v}_{d}-\tilde{v}_{d}^{\prime}\right|, t\left|\tilde{v}_{d+1}-\tilde{v}_{d+1}^{\prime}\right|, \ldots, t\left|\tilde{v}_{4}-\tilde{v}_{4}^{\prime}\right|\right) \gtrsim \delta .
$$

Let $\mathcal{L}_{1} \subset \Sigma_{\delta}\left(Z \cap N_{t}(H)\right)$ be a set of lines pointing in $\delta$-separated directions. We will "thin out" the set of lines in $\mathcal{L}_{1}$ by a factor of $t^{-1}$ in each of the $d-1$ directions $e_{2}, \ldots, e_{d}$. More precisely, let $\mathcal{L}_{2} \subset \mathcal{L}_{1}$ be a set of lines with $\left|\mathcal{L}_{2}\right| \gtrsim t^{d-1}\left|\mathcal{L}_{1}\right|$, so that if $\ell, \ell^{\prime} \in \mathcal{L}_{2}$ are distinct, and if $v=v(\ell), v^{\prime}=v\left(\ell^{\prime}\right)$, then at least one of $t\left|v_{2}-v_{2}^{\prime}\right|, \ldots, t\left|v_{d}-v_{d}^{\prime}\right|$, or at least one of $\left.\left|v_{d+1}-v_{d+1}^{\prime}\right|, \ldots,\left|v_{4}-v_{4}^{\prime}\right|\right)$ is $\gtrsim \delta$.

By 85, we have that if $\ell, \ell^{\prime} \in \mathcal{L}_{2}$, then $\angle\left(v(f(\ell)), v\left(f\left(\ell^{\prime}\right)\right)\right) \geq \delta / t$. For each $\ell \in \mathcal{L}_{2}$, we have that

$$
f(\ell) \in \Sigma_{\delta / t}\left(B(0,1) \cap f\left(Z \cap N_{t}(H)\right)\right) .
$$

Applying Proposition 9.1 with $\delta^{\prime}=\delta / t$ and the same values of $D$ and $\varepsilon$ as above, we conclude that

$$
\left|\mathcal{L}_{2}\right| \leq C_{D, \varepsilon}(\delta / t)^{-2-\varepsilon}
$$

and thus

$$
\left|\mathcal{L}_{1}\right| \lesssim C_{D, \varepsilon} t^{3-d+\varepsilon} \delta^{-2-\varepsilon}
$$

Since $\mathcal{L}_{1}$ was an arbitrary set of lines in $\Sigma_{\delta}\left(Z \cap N_{t}(H)\right)$ pointing in $\delta$-separated directions, we obtain (84). 


\subsection{Proof of Proposition 9.1}

Proof. For each fixed value of $D$ and $\varepsilon$, we will prove Proposition 9.1 by induction on $\delta$. Fix $D$ and $\varepsilon$, and suppose that Proposition 9.1 has been proved for all $\delta<\delta^{\prime} \leq 1$; let $C_{D, \varepsilon}$ be the corresponding constant. We will show that if $C_{D, \varepsilon}$ is sufficiently large (depending only on $D$ and $\varepsilon$ ), then Proposition 9.1 holds for $\delta$.

Let $s, \kappa$, and $u$ be parameters that will be determined below; for the impatient reader, $s, \kappa$ and $u$ will be of size roughly $|\log \delta|^{-O_{\varepsilon}}(1)$. Let $P$ be a polynomial of degree at most $D$. Apply Lemma 2.6 to the map $v: \Sigma_{\delta}(Z) \rightarrow S^{3}$ to select a semialgebraic set $\Sigma \subset \Sigma_{\delta}(Z)$ whose lines point in different directions. We have that $\Sigma \subset S^{3}$ is a semi-algebraic set of complexity $O_{D}(1)$, and

$$
\mathcal{E}_{\delta}(v(\Sigma))=\mathcal{E}_{\delta}\left(v\left(\Sigma_{\delta}(Z)\right)\right)
$$

Apply Theorem 1.2 to $Z(P)$ and $\Sigma$, and let $\Sigma_{1}, \Sigma_{2}, \Sigma_{3}, \Sigma_{4}$ be the resulting sets of lines.

1. The lines in $\Sigma_{1}$ can be covered by $O_{D}\left(|\log \delta|^{O(1)} s^{-2}\right)$ rectangular prisms of dimensions $2 \times s \times s \times s$. Applying Lemma 9.1 to each of these prisms, we conclude that

$$
\begin{aligned}
\mathcal{E}_{\delta}\left(v\left(\Sigma_{1}\right)\right) & \lesssim_{D}\left(|\log \delta|^{O(1)} s^{-2}\right) C_{D, \varepsilon} s^{2+\varepsilon} \delta^{-2-\varepsilon} \\
& \leq\left(C_{D}|\log \delta|^{O(1)} s^{\varepsilon}\right) C_{D, \varepsilon} \delta^{-2-\varepsilon} .
\end{aligned}
$$

Thus there exist constants $c_{1}>0$ and $C_{1}$, depending only on $D$ and $\varepsilon$, so that if we define $s=c_{1}|\log \delta|^{-C_{1}}$ then

$$
\mathcal{E}_{\delta}\left(v\left(\Sigma_{1}\right)\right) \leq \frac{C_{D, \varepsilon}}{4} \delta^{-2-\varepsilon}
$$

2. The lines in $\Sigma_{2}$ can be covered by $O_{D}\left((|\log \delta| / s)^{O(1)} u^{-1}\right)$ rectangular prisms of dimensions $2 \times 2 \times u \times u$. Applying Lemma 9.1 to each of these prisms, we conclude that

$$
\begin{aligned}
\mathcal{E}_{\delta}\left(v\left(\Sigma_{2}\right)\right) & \lesssim_{D}\left((|\log \delta| / s)^{O(1)} u^{-1}\right) C_{D, \varepsilon} u^{1+\varepsilon} \delta^{-2-\varepsilon} \\
& \leq\left(C_{D}|\log \delta|^{O(1)} s^{-O(1)} u^{\varepsilon}\right) C_{D, \varepsilon} \delta^{-2-\varepsilon}
\end{aligned}
$$

Thus there exist constants $c_{2}>0$ and $C_{2}$, depending only on $D$ and $\varepsilon$ (also on $c_{1}$ and $C_{1}$, but this depends only on $D$ and $\left.\varepsilon\right)$, so that if we define $u=c_{2}|\log \delta|^{-C_{2}}$ then

$$
\mathcal{E}_{\delta}\left(v\left(\Sigma_{2}\right)\right) \leq \frac{C_{D, \varepsilon}}{4} \delta^{-2-\varepsilon}
$$


3. The lines in $\Sigma_{3}$ can be covered by $O_{D}\left(|\log \delta|^{O(1)}\right)$ rectangular prisms of dimensions $2 \times 2 \times 2 \times \kappa$. Applying Lemma 9.1 to each of these prisms, we conclude that

$$
\begin{aligned}
\mathcal{E}_{\delta}\left(v\left(\Sigma_{3}\right)\right) & \lesssim_{D}\left(|\log \delta|^{O(1)}\right) C_{D, \varepsilon} \kappa^{\varepsilon} \delta^{-2-\varepsilon} \\
& \leq\left(C_{D}|\log \delta|^{O(1)} \kappa^{\varepsilon}\right) C_{D, \varepsilon} \delta^{-2-\varepsilon}
\end{aligned}
$$

Thus there exist constants $c_{3}>0$ and $C_{3}$, depending only on $D$ and $\varepsilon$, so that if we define $\kappa=c_{3}|\log \delta|^{-C_{3}}$ then

$$
\mathcal{E}_{\delta}\left(v\left(\Sigma_{3}\right)\right) \leq \frac{C_{D, \varepsilon}}{4} \delta^{-2-\varepsilon}
$$

4. Finally, there exists a set $\Sigma_{4}^{\prime} \subset \Sigma_{4}$ and a quadratic polynomial $Q$ so that $\mathcal{E}_{\delta}\left(\Sigma_{4}^{\prime}\right){ }_{D}(s \kappa u /|\log \delta|)^{O(1)} \mathcal{E}_{\delta}\left(\Sigma_{4}\right)$, and for every line $\ell^{\prime} \in \Sigma_{4}^{\prime}$, there is a line $\ell$ contained in $Z(Q)$ with $\operatorname{dist}\left(\ell, \ell^{\prime}\right) \lesssim_{D}(|\log \delta| /(s \kappa u))^{O(1)} \delta$. Note that for every quadratic polynomial $Q$, we have

$\mathcal{E}_{\delta}\left(\left\{\ell \in \mathcal{L}:\right.\right.$ there exists $\ell^{\prime} \subset Z(Q)$ with $\left.\left.\operatorname{dist}\left(\ell, \ell^{\prime}\right) \lesssim_{D}(s \kappa u)^{-O(1)} \delta\right\}\right) \lesssim_{D}(s \kappa u)^{-O(1)} \delta^{-2}$,

and thus

$$
\mathcal{E}_{\delta}\left(v\left(\Sigma_{4}^{\prime}\right)\right) \lesssim_{D}(|\log \delta| / s \kappa u)^{-O(1)} \delta^{-2}
$$

SO

$$
\mathcal{E}_{\delta}\left(v\left(\Sigma_{4}\right)\right) \lesssim_{D}|\log \delta|^{O(1)}(s \kappa u)^{-O(1)} \delta^{-2} .
$$

Thus there exist constants $c_{4}>0$ and $C_{4}$, depending only on $D$ and $\varepsilon$ (also on $\left.c_{1}, c_{2}, c_{3}, C_{1}, C_{2}, C_{3}\right)$, but these in turn only depend on $D$ and $\varepsilon$ ) so that

$$
\mathcal{E}_{\delta}\left(v\left(\Sigma_{4}\right)\right) \leq c_{4}|\log \delta|^{-C_{4}} \delta^{-2}
$$

If $C_{D, \varepsilon}$ is sufficiently large (depending only on $D$ and $\varepsilon$ ), then $c_{4}|\log \delta|^{-C_{4}} \leq \frac{C_{D, \varepsilon}}{4} \delta^{-2-\varepsilon}$, and thus

$$
\mathcal{E}_{\delta}\left(v\left(\Sigma_{4}\right)\right) \leq \frac{C_{D, \varepsilon}}{4} \delta^{-2-\varepsilon} .
$$

Combining 86), 87), 88) and (89), we conclude that

$$
\mathcal{E}_{\delta}\left(v\left(\Sigma_{\delta}(Z)\right)\right) \leq C_{D, \varepsilon} \delta^{-2-\varepsilon} .
$$

This completes the induction and concludes the proof. 


\section{Improved Kakeya estimates in $\mathbb{R}^{4}$}

In this section, we will show that Corollary 1.2 implies Theorem 1.3 . First, we will recall several definitions from [11]. Throughout this section, a $\delta$-tube is the $\delta$-neighborhood of a unit line segment contained in $B(0,1)$.

Definition 10.1 (Two-ends condition). Let $\mathbb{T}$ be a set of $\delta$-tubes. For each $T \in \mathbb{T}$, let $Y(T) \subset T$. We say that the tubes in $\mathbb{T}$ satisfy the two-ends condition with exponent $\rho$ and error $\alpha$ if for all $T \in \mathbb{T}$ and for all balls $B(x, r)$ of radius $r$, we have

$$
|Y(T) \cap B(x, r)| \leq \alpha r^{\rho}|Y(T)|
$$

Definition 10.2 (Robust transversality condition). Let $\mathbb{T}$ be a set of $\delta$-tubes. For each $T \in \mathbb{T}$, let $Y(T) \subset T$. We say that $\mathbb{T}$ is $\beta$-robustly transverse if for all $x \in \mathbb{R}^{4}$ and all vectors $v$, we have

$$
|\{T \in \mathbb{T}: x \in Y(T), \angle(T, v)<\beta\}| \leq \frac{1}{100}|\{T \in \mathbb{T}: x \in Y(T)\}| .
$$

Definition 10.3. Let $\mathbb{T}$ be a set of $\delta$-tubes. We say that $\mathbb{T}$ satisfies the linear Wolff axioms if for every rectangular prism $R$ of dimensions $1 \times t_{1} \times t_{2} \times t_{3}$, at most $100 t_{1} t_{2} t_{3} \delta^{-3}$ tubes from $\mathbb{T}$ can be contained in $R$.

With these two definitions, we can now state Proposition 6.2 from [11]:

Proposition 10.1 ( [11], Proposition 6.2). For each $\epsilon>0$ and $\rho>0$ there exist constants $c, C$, and $D$ so that the following holds. Let $\mathbb{T}$ be a set of $\delta$-tubes in $\mathbb{R}^{4}$. Suppose that $\mathbb{T}$ satisfies the linear Wolff axioms. Suppose furthermore that for every integer $1 \leq E \leq D$, for every polynomial $P \in \mathbb{R}\left[x_{1}, x_{2}, x_{3}, x_{4}\right]$ of degree $E$, for every ball $B(x, r)$ of radius $r$, and for every $w>0$, we have

$$
\left|\left\{T \in \mathbb{T}: T \cap B(x, r) \subset N_{10 \delta}(Z)\right\}\right| \leq K_{E, w} r^{-1} \delta^{-2-w} .
$$

For each $T \in \mathbb{T}$, let $Y(T) \subset T$ with $\lambda \leq|Y(T)| /|T| \leq 2 \lambda$. Suppose that $(\mathbb{T}, Y)$ is $s$-robustly transverse and that each tube $T \in \mathbb{T}$ satisfies the two-ends condition with exponent $\rho$ and error $\alpha$. Then

$$
\left|\bigcup_{T \in \mathbb{T}} Y(T)\right| \geq c_{s} \alpha^{-C} \lambda^{3+1 / 28} K^{-1} \delta^{1-1 / 28+\epsilon}\left(\delta^{3}|\mathbb{T}|\right)
$$

where $K=\max _{1 \leq E \leq D} K_{E}$. 
Observe that inequality $(92)$ is a re-scaled version of Theorem 1.2 . Indeed, Theorem 1.2 asserts that if $\mathbb{T}$ is a set of $\delta$-tubes pointing in $\delta$-separated directions, then for every polynomial $P \in \mathbb{R}\left[x_{1}, x_{2}, x_{3}, x_{4}\right]$ of degree $E$, for every ball $B(x, r)$ of radius $r$, and for every $w>0$, we have

$$
\left|\left\{T \in \mathbb{T}: T \cap B(x, r) \subset N_{10 \delta}(Z)\right\}\right| \leq C_{E, w} r^{-3}(\delta / r)^{-2-w} \leq C_{E, w} r^{-1} \delta^{-2-w} .
$$

Since every set of $\delta$-tubes pointing in $\delta$-separated directions satisfies the linear Wolff axioms, we obtain the following variant of Proposition 10.1 .

Proposition 10.2. For each $\epsilon>0$ and $\rho>0$ there exist constants $c$ and $C$ so that the following holds. Let $\mathbb{T}$ be a set of $\delta$-tubes in $\mathbb{R}^{4}$ pointing in $\delta$-separated directions. For each $T \in \mathbb{T}$, let $Y(T) \subset T$ with $\lambda \leq|Y(T)| /|T| \leq 2 \lambda$. Suppose that $(\mathbb{T}, Y)$ is $s$-robustly transverse and that each tube $T \in \mathbb{T}$ satisfies the two-ends condition with exponent $\rho$ and error $\alpha$. Then

$$
\left|\bigcup_{T \in \mathbb{T}} Y(T)\right| \geq c_{s} c \alpha^{-C} \lambda^{3+1 / 28} \delta^{1-1 / 28+\epsilon}\left(\delta^{3}|\mathbb{T}|\right) .
$$

Finally, Theorem 1.3 follows from Proposition 10.2 using the standard two-ends reduction and bilinear reduction. See e.g. [11, Section 2] for details.

\section{References}

[1] S. Barone and S. Basu. On a real analog of Bezout inequality and the number of connected components of sign conditions. Proc. London Math. Soc. 112(1):115$145,2016$.

[2] J. Bochnak, M. Coste and M. Roy. Real algebraic geometry. Springer-Verlag, Berlin. 1998.

[3] Y. Brudnyi and I. Ganzburg. On an extremal problem for polynomials in $n$ variables. Math. USSR Izvestijia 7:345-356. 1973.

[4] A. Córdoba. The Kakeya maximal function and the spherical summation multiplier. Am. J. Math. 99:1-22. 1977.

[5] C. Demeter. On the restriction theorem for paraboloid in $\mathbb{R}^{4}$. arXiv:1701.03523. 2017. 
[6] Z. Dvir. On the size of Kakeya sets in finite fields. J. Amer. Math. Soc. 22:10931097. 2009.

[7] Z. Dvir, S. Kopparty, S. Saraf, and M. Sudan. Extensions to the method of multiplicities, with applications to Kakeya sets and mergers. SIAM J. Comput. 42(6):2305-2328. 2013.

[8] C. Fefferman. Inequalities for strongly singular convolution operators. Acta Math. 124:9-36, 1970.

[9] L. Guth. Restriction estimates using polynomial partitioning. J. Amer. Math. Soc. 29(2):371-413, 2016

[10] L. Guth. Restriction estimates using polynomial partitioning II. arXiv:1603.04250, 2016.

[11] L. Guth, J. Zahl. Polynomial Wolff axioms and Kakeya-type estimates in $\mathbb{R}^{4}$. To appear, Proc. London Math. Soc. arXiv:1701.07045. 2017.

[12] N. Katz, I. Łaba, T. Tao. An improved bound on the Minkowski dimension of Besicovitch sets in $\mathbb{R}^{3}$. Ann. of Math. 152: 383-446, 2000.

[13] N. Katz, K. Rogers. On the polynomial Wolff axioms. arXiv:1802.09094. 2018.

[14] N. Katz, J. Zahl. An improved bound on the Hausdorff dimension of Besicovitch sets in $\mathbb{R}^{3}$. arXiv:1704.07210, 2017.

[15] I. Łaba, T. Tao. An improved bound for the Minkowski dimension of Besicovitch sets in medium dimension. Geom. Funct. Anal. 11(4):773-806. 2001.

[16] J. Milnor. On the Betti numbers of real varieties. Proc. Amer. Math. Soc. 15(2): 275-280. 1964.

[17] E. Rogora. Varieties with many lines. Manuscripta Math. 82(1): 207-226. 1994.

[18] B. Segre. Sulle $V_{n}$ aventi piú di $\infty^{n-k} S_{k}$. Rendiconti dell'accademia nazionale dei Lincei. Vol. V, note I e II. 1948.

[19] F. Severi. Intorno ai punti doppi impropri di una superficie generale dello spazio a quattro dimensioni, e a'suoi punti tripli apparenti. Rend. circ. mat. Palermo 15(1): 33-51. 1901. 
[20] M. Sharir and N. Solomon. Incidences between points and lines in $\mathbb{R}^{4}$. Disc. Comput. Geom. 57(3); 702-756. 2017.

[21] T. Tao. A new bound for finite field Besicovitch sets in four dimensions. Pacific J. Math. 222: 43-57. 2005

[22] T. Wolff. An improved bound for Kakeya type maximal functions. Rev. Mat. Iberoam. 11(3): 651-674. 1995.

[23] R. Wongkew. Volumes of tubular neighbourhoods of real algebraic varieties. Pacific J. Math. 159:177-184. 2003.

[24] Y. Yomdin and G. Comte, Tame geometry with application in smooth analysis. Springer-Verlag, Berlin, 2004.

[25] A. Zygmund. On Fourier coefficients and transforms of functions of two variables. Studia Math. 50:189-201. 1974. 